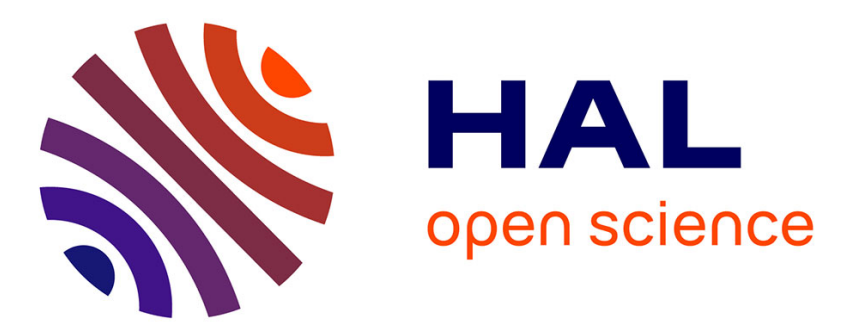

\title{
On the controllability of an advection-diffusion equation with respect to the diffusion parameter: Asymptotic analysis and numerical simulations
}

\author{
Youcef Amirat, Arnaud Munch
}

\section{- To cite this version:}

Youcef Amirat, Arnaud Munch. On the controllability of an advection-diffusion equation with respect to the diffusion parameter: Asymptotic analysis and numerical simulations. Acta Mathematicae Applicatae Sinica, In press. hal-01823289

\section{HAL Id: hal-01823289 \\ https://hal.science/hal-01823289}

Submitted on 25 Jun 2018

HAL is a multi-disciplinary open access archive for the deposit and dissemination of scientific research documents, whether they are published or not. The documents may come from teaching and research institutions in France or abroad, or from public or private research centers.
L'archive ouverte pluridisciplinaire HAL, est destinée au dépôt et à la diffusion de documents scientifiques de niveau recherche, publiés ou non, émanant des établissements d'enseignement et de recherche français ou étrangers, des laboratoires publics ou privés. 


\title{
On the controllability of an advection-diffusion equation with respect to the diffusion parameter: Asymptotic analysis and numerical simulations
}

\author{
Youcef Amirat* $\quad$ ARnaud MÜNCH ${ }^{\dagger}$
}

June 25, 2018

Paper dedicated to Professor Philippe G. Ciarlet on the occasion of his $80^{\text {th }}$ birthday.

\begin{abstract}
The advection-diffusion equation $y_{t}^{\varepsilon}-\varepsilon y_{x x}^{\varepsilon}+M y_{x}^{\varepsilon}=0,(x, t) \in(0,1) \times(0, T)$ is null controllable for any strictly positive values of the diffusion coefficient $\varepsilon$ and of the controllability time $T$. We discuss here the behavior of the cost of control when the coefficient $\varepsilon$ goes to zero, according to the values of $T$. It is actually known that this cost is uniformly bounded with respect to $\varepsilon$ if $T$ is greater than a minimal time $T_{M}$, with $T_{M}$ in the interval $[1,2 \sqrt{3}] / M$ for $M>0$ and in the interval $[2 \sqrt{2}, 2(1+\sqrt{3})] /|M|$ for $M<0$. The exact value of $T_{M}$ is however unknown.

We investigate in this work the determination of the minimal time $T_{M}$ employing two distincts but complementary approaches. In a first one, we numerically estimate the cost of controllability, reformulated as the solution of a generalized eigenvalue problem for the underlying control operator, with respect to the parameter $T$ and $\varepsilon$. This allows notably to exhibit the structure of initial data leading to large costs of control. At the practical level, this evaluation requires the non trivial and challenging approximation of null controls for the advection-diffusion equation. In the second approach, we perform an asymptotic analysis, with respect to the parameter $\varepsilon$, of the optimality system associated to the control of minimal $L^{2}$-norm. The matched asymptotic expansion method is used to describe the multiple boundary layers.
\end{abstract}

Key words: Numerical approximation, space-time variational formulation, Asymptotic analysis, Boundary layers, Null controllability.

\section{Introduction}

Let $L>0, T>0$ and $Q_{T}:=(0, L) \times(0, T)$. This work is concerned with the scalar advection-diffusion equation

$$
\left\{\begin{array}{lr}
y_{t}^{\varepsilon}-\varepsilon y_{x x}^{\varepsilon}+M y_{x}^{\varepsilon}=0, & (x, t) \in Q_{T}, \\
y^{\varepsilon}(0, t)=v^{\varepsilon}(t), y^{\varepsilon}(L, t)=0, & t \in(0, T), \\
y^{\varepsilon}(x, 0)=y_{0}^{\varepsilon}(x), & x \in(0, L) .
\end{array}\right.
$$

The parameter $\varepsilon>0$ is the diffusion coefficient while the real $M$ is the transport coefficient; $v^{\varepsilon}=v^{\varepsilon}(t)$ is the control function in $L^{2}(0, T), y_{0}^{\varepsilon} \in H^{-1}(0, L)$ is the initial data and $y^{\varepsilon}=y^{\varepsilon}(x, t)$ is the associated state.

For any $y_{0}^{\varepsilon} \in H^{-1}(0, L)$ and $v^{\varepsilon} \in L^{2}(0, T)$, there exists exactly one solution $y^{\varepsilon}$ to $(1)$, with the regularity $y^{\varepsilon} \in L^{2}\left(Q_{T}\right) \cap C\left([0, T] ; H^{-1}(0, L)\right)$ (see [18, Prop. 2.2]). Accordingly, for any final time $T>0$, the associated

\footnotetext{
*Laboratoire de Mathématiques Blaise Pascal, Université Clermont Auvergne, UMR CNRS 6620, Campus universitaire des Cézeaux, 3, place Vasarely, 63178, Aubière, France. E-mail: youcef .amirat@uca.fr.

†Laboratoire de Mathématiques Blaise Pascal, Université Clermont Auvergne, UMR CNRS 6620, Campus universitaire des Cézeaux, 3, place Vasarely, 63178, Aubière, France. E-mail: arnaud.munch@uca.fr.
} 
null controllability problem at time $T>0$ is the following: for each $y_{0}^{\varepsilon} \in H^{-1}(0, L)$, find $v \in L^{2}(0, T)$ such that the corresponding solution to (1) satisfies

$$
y^{\varepsilon}(\cdot, T)=0 \text { in } H^{-1}(0, L) .
$$

For any $T>0, M \in \mathbb{R}$ and $\varepsilon>0$, the null controllability for the parabolic type equation (1) holds true (see [20, 27]). We therefore introduce the non-empty set of null controls

$$
\left.\mathcal{C}\left(y_{0}^{\varepsilon}, T, \varepsilon, M\right):=\left\{(y, v): v \in L^{2}(0, T) ; y \text { solves } 11\right) \text { and satisfies }(2)\right\} .
$$

We are mainly concerned with the asymptotic behavior of null controls for (1) when the coefficient $\varepsilon$ becomes small. System (1) can be seen as a simple example of complex models where the diffusion coefficient is very small compared to the others. We have notably in mind the Stokes system where $\varepsilon$ stands for the viscosity coefficient. One may also think of a parabolic regularization of a system of conservation laws. Precisely, we are interested with the control of minimal $L^{2}$-norm and define for any $\varepsilon>0$ the cost of control as follows:

Definition 1.1 For all $\varepsilon>0, T>0, M \in \mathbb{R}$, we define

$$
K(\varepsilon, T, M):=\sup _{\left\|y_{0}^{\varepsilon}\right\|_{L^{2}(0, L)}=1}\left\{\min _{u \in \mathcal{C}\left(y_{0}^{\varepsilon}, T, \varepsilon, M\right)}\|u\|_{L^{2}(0, T)}\right\} .
$$

For the sake of simplicity, we consider the supremum over initial conditions in $L^{2}(0, L)$. A similar definition holds true with the space $H^{-1}(0, L)$. The cost of control $K(\varepsilon, T, M)$ is the norm of the (linear) operator $y_{0}^{\varepsilon} \rightarrow v_{H U M}^{\varepsilon}$ where $v_{H U M}^{\varepsilon}$ is the control of minimal $L^{2}$-norm.

Definition 1.2 Let

$$
T_{M}:=\inf \{T>0: \exists C>0 \quad \text { s.t. } \quad \forall \varepsilon>0, K(\varepsilon, T, M)<C\},
$$

so that (1) is uniformly controllable with respect to $\varepsilon$ if and only if $T \geq T_{M}$.

For $\varepsilon=0$, the system (1) degenerates into a transport equation and is uniformly controllable as soon as $T$ is large enough, according to the speed $|M|$ of transport, precisely as soon as $T \geq L /|M|$.

Definition 1.3 For all $L>0$ and $M \in \mathbb{R}^{\star}$, we denote

$$
T^{\star}:=\frac{L}{|M|} .
$$

Obviously, if $T \geq T^{\star}$, the zero function is a null control - and so the control of minimal $L^{2}$-norm - for the transport equation. Accordingly, $K(0, T, M)=0$ for any $T \geq T^{\star}$.

On the other hand, the behavior of $K(\varepsilon, T, M)$ as $\varepsilon \rightarrow 0$ is more involved and has been the subject of several recent works. We may expect that the $\operatorname{cost} K(\varepsilon, T, M)$ goes to zero as $\varepsilon \rightarrow 0$ as soon as $T \geq T^{\star}$ and therefore that $T_{M}=T^{\star}$. But, at least for $M<0$, this is false since, as shown in [29, the cost $K(\varepsilon, T, M)$ blows up exponentially as $\varepsilon \rightarrow 0^{+}$for any $T<2(1+\sqrt{3}) T^{\star}$. This is a priori a surprising and non expected result. There is actually a kind of balance between the term $-\varepsilon y_{x x}^{\varepsilon}$ which favors the diffusion (and so the null controllability) for $\varepsilon$ large and the term $M y_{x}^{\varepsilon}$ which enhances the complete transport of the solution out of the domain $(0, L)$ for $\varepsilon$ small. Precisely, for any $t$ larger than $T^{\star}$, the $L^{2}$-norm $\left\|y^{\varepsilon}(\cdot, t)\right\|_{L^{2}(0, L)}$ is of the order $e^{-\frac{1}{\varepsilon}\left(t-T^{\star}\right)^{2}}$, exponentially small but non zero. This is the effect of the transport term. On the other hand, the cost of null control for the heat equation with initial condition of magnitude one and diffusion $\varepsilon$ is exponentially large, of the order $e^{\frac{L}{\varepsilon T}}$. The time $T_{M}$ is therefore related to the minimal time beyond which the transport of the solution is enough to compensate the cost of control to zero of the remainder.

One may tackle this problem and the determination of the minimal uniform controllability time $T_{M}$ using at least two distincts approaches. A first one, discussed in [33], consists in approximating numerically the cost $K(\varepsilon, T, M)$ for various values of $\varepsilon$ and $T>0$, the ratio $L / M$ being fixed. This can be done by reformulating the cost as the solution of a generalized eigenvalue problem for the control operator associated to (1). This latter problem requires the approximation of the control of minimal $L^{2}$-norm, a delicate issue for small values of $\varepsilon$. 
A second approach, discussed in [1, consists in performing, in the spirit of [28, an asymptotic analysis of the optimality system associated to the control of minimal $L^{2}$-norm. In spite of the apparent simplicity of the system (1), such analysis is not straightforward because, as $\varepsilon$ goes to zero, the direct and adjoint solutions exhibit boundary layers in the transition parabolic to hyperbolic. For example, for $M>0$, the solution $y^{\varepsilon}$ exhibits a first boundary layer of size $\mathcal{O}(\varepsilon)$ at $x=L$ and a second boundary layer of size $\mathcal{O}(\sqrt{\varepsilon})$ along the characteristic $\left\{(x, t) \in Q_{T}, L x-M t=0\right\}$. A third singular behavior due to the initial condition $y_{0}^{\varepsilon}$ occurs for $y^{\varepsilon}$ in the neighborhood of the points $\left(x_{0}, t_{0}\right)=(0,0)$ and $\left(x_{1}, t_{1}\right)=(L, 0)$.

We review in this paper existing results as well as well recent contributions based on the two previous mentioned approaches. In Section 2, we preliminary recall some important facts about the system (1) and some corresponding existing controllability results. In particular, we enhance the fact that, for this system, the difference between null and approximate controllability becomes very important as $\varepsilon$ goes to zero. Then, in Section 3, we introduce and discuss, mainly formally, a method allowing to approximate numerically the cost $K$ for relatively large values of $\varepsilon$. Then, in Section 4, we investigate in a rigorous way the asymptotic analysis of the problem with respect to $\varepsilon$. We conclude in Section 6 with some perspectives.

Along the text, we shall use the following notations:

$$
L_{\varepsilon} y:=y_{t}-\varepsilon y_{x x}+M y_{x}, \quad L_{\varepsilon}^{\star} \varphi:=-\varphi_{t}-\varepsilon \varphi_{x x}-M \varphi_{x} .
$$

\section{Asymptotic properties and overview of controllability results}

We overview in this section some qualitative results related to the system (1) as well as controllability results. We start with two remarks.

Remark 1 If $M=0,(1)$ is simply the heat equation, uniformly controllable with respect to $T>0$ and $y_{0}^{\varepsilon} \in$ $H^{-1}(0, L)$. By making the change of variable $\tilde{t}=\varepsilon t$, we easily obtain the equality $K(\varepsilon, T, 0)=\varepsilon^{-1 / 2} K(1, \varepsilon T, 0)$ for all $\varepsilon>0$ and $T>0$. Moreover, the cost of control for the heat equation in arbitrarily small time has been studied in various places (see [31] and the references therein) and is as follows: $K(1, T, 0) \sim_{T \rightarrow 0^{+}} e^{\frac{\kappa L}{T}}$ for some $\kappa \in(1 / 2,3 / 4)$. Gathering these two estimates, we deduce that

$$
K(\varepsilon, T, 0) \sim_{\varepsilon \rightarrow 0^{+}} \varepsilon^{-1 / 2} e^{\frac{\kappa L^{2}}{\varepsilon T}}, \quad \kappa \in(1 / 2,3 / 4)
$$

and conclude that, for $M=0$, the cost of control defined by (3) blows up exponentially with respect to the diffusion coefficient. We may write $T_{0}=+\infty$. The open question in the case $M=0$ is the value of the exponential rate $\kappa$ known to be in the range $(1 / 2,3 / 4)$.

In view of (6), we assume in the sequel that $M \neq 0$, for which the situation is different as it also depends on the value of the controllability time $T$. It is also useful to recall the following fact, which can be obtained by standard duality arguments.

Remark 2 The controllability cost is related to the observability constant $C_{\text {obs }}(\varepsilon, T, M)$ which appears in the observability inequality for (8)

$$
\left\|\varphi^{\varepsilon}(\cdot, 0)\right\|_{L^{2}(0, L)} \leq C_{o b s}(\varepsilon, T, M)\left\|\varepsilon \varphi_{x}^{\varepsilon}(0, \cdot)\right\|_{L^{2}(0, T)}, \quad \forall \varphi_{T}^{\varepsilon} \in H_{0}^{1}(0, L) \cap H^{2}(0, L),
$$

defined by

$$
C_{o b s}(\varepsilon, T, M):=\sup _{\varphi_{T}^{\varepsilon} \in H_{0}^{1}(0, L)} \frac{\left\|\varphi^{\varepsilon}(\cdot, 0)\right\|_{L^{2}(0, L)}}{\left\|\varepsilon \varphi_{x}^{\varepsilon}(0, \cdot)\right\|_{L^{2}(0, T)}},
$$

where $\varphi^{\varepsilon}$ solves the adjoint system

$$
\left\{\begin{array}{lc}
\varphi_{t}^{\varepsilon}+\varepsilon \varphi_{x x}^{\varepsilon}+M \varphi_{x}^{\varepsilon}=0, & (x, t) \in Q_{T}, \\
\varphi^{\varepsilon}(0, t)=\varphi^{\varepsilon}(L, t)=0, & t \in(0, T), \\
\varphi^{\varepsilon}(x, T)=\varphi_{T}^{\varepsilon}(x), & x \in(0, L) .
\end{array}\right.
$$

Precisely, we get that $K(\varepsilon, T, M)=C_{o b s}(\varepsilon, T, M)$ (see [14], Remark 2.98). The observability inequality is the main tool to prove the null controllability of (1). 
The first result we recall is due to J-.M. Coron and S. Guerrero and concerns the asymptotic behavior of the direct solution $y^{\varepsilon}$, with respect to $\varepsilon$, for $v^{\varepsilon}$ given in $L^{2}(0, T)$.

Theorem 2.1 (Coron-Guerrero [15]) Let $T>0, M \in \mathbb{R}^{\star}, y_{0} \in L^{2}(0, L)$, independent of $\varepsilon$. Let $\left(v^{\varepsilon}\right)_{(\varepsilon)}$ be a sequence of functions in $L^{2}(0, T)$ such that for some $v \in L^{2}(0, T), v^{\varepsilon} \rightarrow v$ in $\overline{L^{2}(0, T) \text {, as } \varepsilon \rightarrow 0^{+}}$. For $\varepsilon>0$, let us denote by $y^{\varepsilon} \in C\left([0, T] ; H^{-1}(0, L)\right)$ the weak solution of

$$
\left\{\begin{array}{lr}
y_{t}^{\varepsilon}-\varepsilon y_{x x}^{\varepsilon}+M y_{x}^{\varepsilon}=0, & (x, t) \in Q_{T}, \\
y^{\varepsilon}(0, t)=v^{\varepsilon}(t), y^{\varepsilon}(L, t)=0, & t \in(0, T), \\
y^{\varepsilon}(x, 0)=y_{0}(x), & x \in(0, L) .
\end{array}\right.
$$

Then, $y^{\varepsilon} \rightarrow y$ in $L^{2}\left(Q_{T}\right)$ as $\varepsilon \rightarrow 0^{+}$where $y \in C\left([0, T] ; L^{2}(0, L)\right)$ is the weak solution of

$$
\left\{\begin{array}{lrl}
y_{t}+M y_{x}=0, & (x, t) \in Q_{T}, \\
y(0, t)=v(t), \quad \text { if } \quad M>0 & t \in(0, T), \\
y(L, t)=0, \quad \text { if } \quad M<0 & t \in(0, T), \\
y(x, 0)=y_{0}(x), & x \in(0, L) .
\end{array}\right.
$$

The fact that the limit weak solution solves the transport equation is quite expected: it is however crucial to note that this theorem assumes that the initial condition does not depend on the parameter $\varepsilon$. As a consequence of this result, if $T<T^{\star}$, one may exhibit initial data $y_{0}^{\varepsilon}$ for which the corresponding control cost is not bounded with respect to $\varepsilon$ :

Corollary 2.1 If $T<T^{\star}, \lim _{\varepsilon \rightarrow 0} K(\varepsilon, T, M)=\infty$. Consequently, $T_{M} \geq T^{\star}$.

Proof. Assume that $K(\varepsilon, T, M) \nrightarrow \rightarrow+\infty$. There exists $\left(\varepsilon_{n}\right)_{(n \in \mathbb{N})}$ positive tending to 0 such that $\left(K\left(\varepsilon_{n}, T, M\right)\right)_{(n \in \mathbb{N})}$ is bounded. Let $v^{\varepsilon_{n}}$ the optimal control driving $y_{0}$ to 0 at time $T$ and $y^{\varepsilon_{n}}$ the corresponding solution. Let $T_{0} \in\left(T, T^{\star}\right)$. We extend $y^{\varepsilon_{n}}$ and $v^{\varepsilon_{n}}$ by 0 on $\left(T, T_{0}\right)$. From the inequality

$$
\left\|v^{\varepsilon_{n}}\right\|_{L^{2}\left(0, T_{0}\right)}=\left\|v^{\varepsilon_{n}}\right\|_{L^{2}(0, T)} \leq K\left(\varepsilon^{n}, T, M\right)\left\|y_{0}^{\varepsilon}\right\|_{L^{2}(0, L)},
$$

we deduce that $\left(v^{\varepsilon_{n}}\right)_{(n \in \mathbb{N})}$ is bounded in $L^{2}\left(0, T_{0}\right)$, so we extract a subsequence $\left(v^{\varepsilon_{n}}\right)_{(n \in \mathbb{N})}$ such that $v^{\varepsilon_{n}} \rightarrow v$ in $L^{2}\left(0, T_{0}\right)$. We deduce that $y^{\varepsilon_{n}} \rightarrow y$ in $L^{2}\left(Q_{T_{0}}\right)$, solution of the transport equation. Necessarily, $y \equiv 0$ on $(0, L) \times\left(T, T_{0}\right)$ which is a contradiction if the support of $y_{0}$ is such that $\operatorname{supp}\left(y_{0}^{\varepsilon}\right) \cap(0, L-T|M|) \neq 0(M>0)$ or $\operatorname{supp}\left(y_{0}^{\varepsilon}\right) \cap(L-T|M|, L) \neq 0(M<0)$.

Corollary 2.1 thus provides a lower bound for $T_{M}$. On the other hand, since the limit system 10 is uniformly null controllable (with null controls) if and only if $T$ is greater than $T^{\star}$, we expect that $T_{M}=T^{\star}$ and moreover that $\lim _{\varepsilon \rightarrow 0} K(\varepsilon, T, M)=0$ as soon as $T \geq T^{\star}$. As we will see, this is not so simple since the cost of control may be achieved by an initial data dependent on $\varepsilon$, a situation not covered by Theorem 2.1. The following result makes precise the behavior of the $\operatorname{cost} K(\varepsilon, T, M)$. We also mention [22] for a multi-dimensional case.

Theorem 2.2 (Coron-Guerrero [15]) If $M>0$ and $T<T^{\star}$, then there exist constants $c, C>0$ such that $K(\varepsilon, T, M) \geq C e^{c / \varepsilon}$ for all $\varepsilon>0$. Similarly, if $M<0$ and $T<2 T^{\star}$, then there exist constants $c, C>0$ such that $K(\varepsilon, T, M) \geq C e^{c / \varepsilon}, c, C>0$ for all $\varepsilon>0$.

Therefore, if $T<T^{\star}$, the control cost blows up exponentially as $\varepsilon$ goes to zero (as for the heat equation, see Remark 11. The theorem also clearly states that the case $M<0$ is much more singular and that $T_{M} \geq 2 T^{\star}$ : heuristically, when $M$ is negative, as $\varepsilon \rightarrow 0$, the solution $y^{\varepsilon}$ is mainly transported from the right to the left parallel to the first characteristic, and therefore somehow acts "against" the control, active at the left extremity $x=0$. For the same reason, for $M>0$, the transport term $M y_{x}$ "helps" the control. These lower bounds are obtained by considering the following condition (the first mode associated to the advection-diffusion operator)

$$
y_{0}^{\varepsilon}(x)=K_{\varepsilon} e^{\frac{M x}{2 \varepsilon}} \sin \left(\frac{\pi x}{L}\right), \quad x \in(0, L)
$$


with $K_{\varepsilon}=\mathcal{O}\left(\varepsilon^{-3 / 2} e^{-\frac{M}{2 \varepsilon}}\right)$ such that $\left\|y_{0}^{\varepsilon}\right\|_{L^{2}(0, L)}=1$ and by estimating the corresponding $L^{2}$-norm of the control of minimal $L^{2}$-norm: precisely, it is obtained, for $M>0$, that

$$
K(\varepsilon, T, M) \geq C_{1} \frac{\varepsilon^{-3 / 2} T^{-1 / 2} L^{2} M^{1 / 2}}{1+L^{3} M^{3} \varepsilon^{-3}} \exp \left(\frac{M}{2 \varepsilon}(L-T M)-\frac{\pi^{2} \varepsilon T}{L^{2}}\right)
$$

while for $M<0$,

$$
K(\varepsilon, T, M) \geq C_{1} \frac{\varepsilon^{-3 / 2} T^{-1 / 2} L^{2} M^{1 / 2}}{1+L^{3}|M|^{3} \varepsilon^{-3}} \exp \left(\frac{|M|}{2 \varepsilon}(2 L-T|M|)-\frac{\pi^{2} \varepsilon T}{L^{2}}\right) .
$$

The initial condition (11) depends on $\varepsilon$, so that the result for $M<0$ does not contradict Theorem 2.1. However, the bounds $T^{\star}$ and $2 T^{\star}$ obtained with such initial condition are quite unexpected. Take for instance $M>0$ so that the initial condition $y_{0}^{\varepsilon}$ given by (11) gets concentrated and supported in the neighborhood of the point $x=L$ (see Figure 1) as $\varepsilon \rightarrow 0$ with $\lim _{\varepsilon \rightarrow 0}\left\|y_{0}^{\varepsilon}\right\|_{L^{\infty}(0, L-\delta)}=0$ for any $\delta>0$. Moreover, this initial condition is transported from the left to the right (again assuming $\varepsilon$ small) so that at any time $t>0$ (not necessarily larger than $T^{\star}$ ) the $L^{2}$-norm of the free solution $\left\|y^{\varepsilon}(\cdot, t)\right\|_{L^{2}(0, L)}$ is exponentially small (see Lemma 2.1 below) with respect to $\varepsilon$. However, according to the bound, if $T$ is lower than $T^{\star}$, the corresponding control cost blows up exponentially with respect to $\varepsilon$. The situation is even worst for $M<0$ (now $y_{0}^{\varepsilon}$ is concentrated at the point $x=0)$ since the property remains true for any $t \in\left(0,2 T^{\star}\right)$ ! Very likely, the worst initial condition (leading to the largest $L^{2}$-norm for the HUM control $\left.v^{\varepsilon}\right)$ is not the one given by (11).

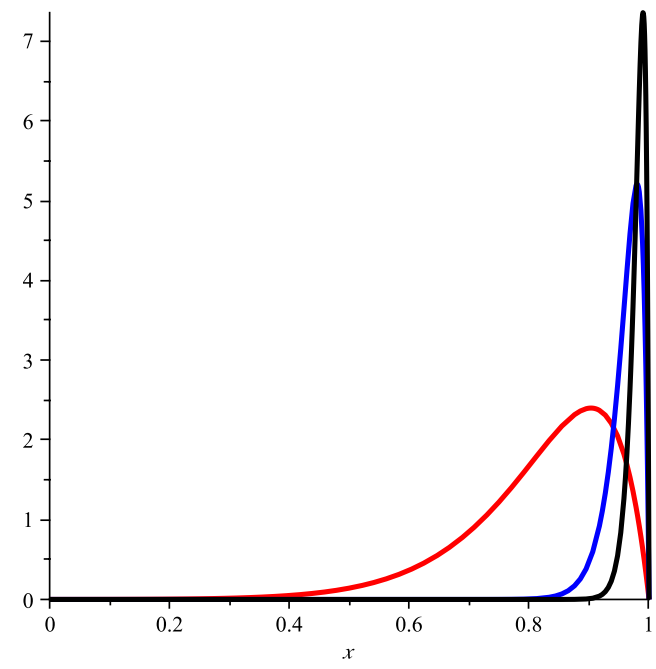

Figure 1: $y_{0}^{\varepsilon}(x)$ for $x \in(0, L)=(0,1)-\varepsilon=5 \times 10^{-2}, \varepsilon=10^{-2}, \varepsilon=5 \times 10^{-3}$.

Before recalling known upper bounds for $T_{M}$, let us consider the behavior of the free solution (i.e. $v^{\varepsilon} \equiv 0$ ) with respect to $\varepsilon$.

Lemma 2.1 Let $\alpha \in(0,1)$. The free solution (i.e. $v^{\varepsilon}=0$ ) satisfies

$$
\left\|y^{\varepsilon}(\cdot, t)\right\|_{L^{2}(0, L)} \leq\left\|y_{0}^{\varepsilon}\right\|_{L^{2}(0, L)} e^{-\frac{L M \alpha^{2}}{4 \varepsilon(1-\alpha)}}, \quad \forall t \geq \frac{T^{\star}}{(1-\alpha)} .
$$

Proof. A proof is given in [15] by extending the system to $x \in \mathbb{R}$ and then by using explicit integral representation of the extended solution. We provide here a simpler one that will be useful in the sequel. We assume $M>0$, the case $M<0$ is similar. We check that $z^{\varepsilon}(x, t)=e^{\frac{-M \alpha x}{2 \varepsilon}} y^{\varepsilon}(x, t)$ solves

$$
\left\{\begin{array}{lr}
z_{t}^{\varepsilon}-\varepsilon z_{x x}^{\varepsilon}+M(1-\alpha) z_{x}^{\varepsilon}-\frac{M^{2}}{4 \varepsilon}\left(\alpha^{2}-2 \alpha\right) z^{\varepsilon}=0, & (x, t) \in Q_{T}, \\
z^{\varepsilon}(0, t)=z^{\varepsilon}(L, t)=0, & t \in(0, T), \\
z^{\varepsilon}(x, 0)=e^{\frac{-M \alpha x}{2 \varepsilon}} y_{0}^{\varepsilon}(x), & x \in(0, L) .
\end{array}\right.
$$


Multiplying the main equation by $z^{\varepsilon}$ and integrating over $(0, L)$ then leads to

$$
\frac{d}{d t}\left\|z^{\varepsilon}(\cdot, t)\right\|_{L^{2}(0, L)}^{2}+2 \varepsilon\left\|z_{x}^{\varepsilon}(\cdot, t)\right\|_{L^{2}(0, L)}^{2}=\frac{M^{2}}{2 \varepsilon}\left(\alpha^{2}-2 \alpha\right)\left\|z^{\varepsilon}(\cdot, t)\right\|_{L^{2}(0, L)}^{2},
$$

and then to the estimate $\left\|z^{\varepsilon}(\cdot, t)\right\|_{L^{2}(0, L)} \leq\left\|z^{\varepsilon}(\cdot, 0)\right\|_{L^{2}(0, L)} e^{\frac{M^{2}}{4 \varepsilon}\left(\alpha^{2}-2 \alpha\right) t}$, equivalently, to

$$
\left\|e^{-\frac{M \alpha x}{2 \varepsilon}} y^{\varepsilon}(\cdot, t)\right\|_{L^{2}(0, L)} \leq\left\|e^{-\frac{M \alpha x}{2 \varepsilon}} y^{\varepsilon}(\cdot, 0)\right\|_{L^{2}(0, L)} e^{\frac{M^{2}}{4 \varepsilon}\left(\alpha^{2}-2 \alpha\right) t} .
$$

Consequently,

$$
\begin{aligned}
\left\|y^{\varepsilon}(\cdot, t)\right\|_{L^{2}(0, L)} & =\left\|e^{\frac{M \alpha x}{2 \varepsilon}} e^{-\frac{M \alpha x}{2 \varepsilon}} y^{\varepsilon}(\cdot, t)\right\|_{L^{2}(0, L)} \leq\left\|e^{\frac{M \alpha x}{2 \varepsilon}}\right\|_{L^{\infty}(0, L)}\left\|e^{-\frac{M \alpha x}{2 \varepsilon}} y^{\varepsilon}(\cdot, t)\right\|_{L^{2}(0, L)} \\
& \leq\left\|e^{\frac{M \alpha x}{2 \varepsilon}}\right\|_{L^{\infty}(0, L)}\left\|e^{-\frac{M \alpha x}{2 \varepsilon}} y^{\varepsilon}(\cdot, 0)\right\|_{L^{2}(0, L)} e^{\frac{M^{2}}{4 \varepsilon}\left(\alpha^{2}-2 \alpha\right) t} \\
& \leq\left\|e^{-\frac{M \alpha x}{2 \varepsilon}} y^{\varepsilon}(\cdot, 0)\right\|_{L^{2}(0, L)} e^{\frac{M \alpha L}{2 \varepsilon}+\frac{M^{2}}{4 \varepsilon}\left(\alpha^{2}-2 \alpha\right) t} \\
& \leq\left\|y^{\varepsilon}(\cdot, 0)\right\|_{L^{2}(0, L)} e^{\frac{M \alpha}{2 \varepsilon}\left(L-M t+\frac{M \alpha t}{2}\right)}
\end{aligned}
$$

using that (recall that $\alpha>0)\left\|e^{\frac{M \alpha x}{2 \varepsilon}}\right\|_{L^{\infty}(0, L)}=e^{\frac{M \alpha L}{2 \varepsilon}}$ and $\left\|e^{-\frac{M \alpha \alpha}{2 \varepsilon}}\right\|_{L^{\infty}(0, L)}=1$. Let now $t \geq \frac{L}{M(1-\alpha)}>\frac{L}{M}$ so that $L-M t+\frac{M \alpha t}{2} \leq-\frac{L \alpha}{2(1-\alpha)}$ and $\frac{M \alpha}{2 \varepsilon}\left(L-M t+\frac{M \alpha t}{2}\right) \leq-\frac{L M \alpha^{2}}{4(1-\alpha)}$. The result follows.

This result shows that the norm $\left\|y^{\varepsilon}(\cdot, t)\right\|_{L^{2}(0, L)}$ of the free solution is exponentially small as soon as $t>T^{\star}$ and exhibits how large is the gap between exact controllability and approximate controllability, for any small enough and fixed $\varepsilon$. Take again $M<0$ and $T \in\left(T^{\star}, 2 T^{\star}\right)$. According to the previous lemma, for any $\delta>0$, there exists $\varepsilon_{0}>0$ such that for all $\varepsilon<\varepsilon_{0},\left\|y^{\varepsilon}(\cdot, T)\right\|_{L^{2}(0, L)} \leq \delta$. The cost of approximate control, says $K_{\delta}(\varepsilon, T, M)$ defined in the same way than $K(\varepsilon, T, M)$ except that $(2)$ is replaced by $\left\|y^{\varepsilon}(\cdot, T)\right\|_{H^{-1}(0, L)} \leq \delta$ is equal to zero, for any $\delta>0$, as soon as $\varepsilon$ is small enough: $\lim _{\varepsilon \rightarrow 0} K_{\delta}(\varepsilon, T, M)=0$ for any $\delta>0$. On the other hand, according to Theorem 2.2, the cost of exact null controllability blows up exponentially. The price to reach the target 0 from any state in the ball $B(0, \delta)=\left\{y \in H^{-1}(0, L),\|y\|_{H^{-1}(0, L)} \leq \delta\right\}$ is arbitrarily large: this is due to the fact that the coefficient of diffusion term $-\varepsilon y_{x x}^{\varepsilon}$, so essential for the controllability property, vanishes. Remark that this kind of balance between transport and diffusion can be seen on the spectrum of the operator $\mathcal{A}: H_{0}^{1}(0, L) \rightarrow H^{-1}(0, L)$ defined by $\mathcal{A}(z)=-\varepsilon z_{x x}+M z_{x}$ given by $\left\{\varepsilon(k \pi)^{2}+M^{2} /(4 \varepsilon)\right\}_{k \in \mathbb{N}}$ associated to the eigenfunctions $\left\{e^{\frac{M x}{2 \varepsilon}} \sin (k \pi x / L)\right\}_{k \in \mathbb{N}}$.

On the other hand, if the controllability time $T>0$ is large enough, then the cost $K(\varepsilon, T, M)$ is bounded uniformly with respect to $\varepsilon$, both for $M>0$ and $M<0$. The following result provides upper bounds for $T_{M}$.

Theorem 2.3 (Coron-Guerrero [15]) Let $\varepsilon>0$. If $M>0$ and $T \geq 4.3 T^{\star}$, then there exists constants $c, C>0$ such that $K(\varepsilon, T, M) \leq C e^{-c / \varepsilon}$. If $M<0$ and $T \geq 57.2 T^{\star}$, then there exists constants $c, C>0$ such that $K(\varepsilon, T, M) \leq C e^{-c / \varepsilon}$.

Consequently, we may summarize the previous results as follows.

Theorem 2.4 (Coron-Guerrero [15]) Let $T_{M}$ be the minimal time for which the control of minimal $L^{2}$ norm is uniformly bounded with respect to $\varepsilon>0$ and $y_{0}^{\varepsilon} \in L^{2}(0, L)$. Then,

$$
T_{M} \in[1,4.3] T^{\star} \quad \text { if } \quad M>0, \quad[2,57.2] T^{\star} \quad \text { if } \quad M<0 .
$$

These bounds have then been improved by O. Glass, P. Lissy and more recently by S. Darde, S. Ervedoza in the positive case $M>0$. Existing results are as follows:

Theorem 2.5 (GLass [21])

$$
T_{M} \in[1,4.2] T^{\star} \quad \text { if } \quad M>0, \quad[2,6.1] T^{\star} \quad \text { if } \quad M<0 .
$$


Theorem 2.6 (Lissy [30])

$$
T_{M} \in[1,2 \sqrt{3}] T^{\star} \quad \text { if } \quad M>0, \quad[2 \sqrt{2}, 2(1+\sqrt{3})] T^{\star} \quad \text { if } \quad M<0 .
$$

We mention that $2 \sqrt{3} \approx 3.46$.

Theorem 2.7 (DARde-ERvedoza [16])

$$
T_{M} \in[1, K] T^{\star} \quad \text { if } \quad M>0, \quad K \approx 3.34
$$

As far as we know, the determination of $T_{M}$, both for $M>0$ and $M<0$, remains an open and nontrivial problem. In the next section, we investigate numerically this issue.

\section{Numerical estimation of the cost of control}

As explained in detail in [33, we may, at least formally, approximate numerically the $\operatorname{cost} K(\varepsilon, T, M)$ with respect to the parameters $\varepsilon, T$ and $M$. We may remark right now that in view of the behavior of the uncontrolled solution given by Lemma 2.1 for $T>T^{\star}$, such numerical approximation will be a priori duable only for not too small values of $\varepsilon$ and $T$ close to $T^{\star}$.

\subsection{Reformulation of the controllability cost $K(\varepsilon, T, M)$}

We reformulate the cost of control $K$ as the solution of a generalized eigenvalue problem involving the control operator (named as the HUM operator by J.-L. Lions for wave type equations). From (3), we write

$$
K^{2}(\varepsilon, T, M)=\sup _{y_{0}^{\varepsilon} \in L^{2}(0, L)} \frac{\left(v^{\varepsilon}, v^{\varepsilon}\right)_{L^{2}(0, T)}}{\left(y_{0}^{\varepsilon}, y_{0}^{\varepsilon}\right)_{L^{2}(0, L)}}
$$

where $v^{\varepsilon}=v\left(y_{0}^{\varepsilon}\right)$ is the null control of minimal $L^{2}(0, T)$-norm for (1) with initial data $y_{0}^{\varepsilon}$. Let us recall that any null control $v$ for (1) satisfies the following characterization

$$
\left(v, \varepsilon \varphi_{x}(0, \cdot)\right)_{L^{2}(0, T)}+\left(y_{0}^{\varepsilon}, \varphi(\cdot, 0)\right)_{L^{2}(0, L)}=0,
$$

for any $\varphi$ solution of the adjoint problem [8] with initial state $\varphi_{T} \in H_{0}^{1}(0, L)$. In particular, the control of minimal $L^{2}$-norm is given by $v^{\varepsilon}=\varepsilon \hat{\varphi}_{x}^{\varepsilon}(0, \cdot)$ in $(0, T)$ where $\hat{\varphi}^{\varepsilon}$ solves $(8)$ associated to the initial $\hat{\varphi}_{T}^{\varepsilon}$, solution of the extremal

$$
\sup _{\varphi_{T}^{\varepsilon} \in H_{0}^{1}(0, L)} J^{\star}\left(\varphi_{T}^{\varepsilon}\right):=\frac{1}{2} \int_{0}^{T}\left(\varepsilon \varphi_{x}^{\varepsilon}(0, \cdot)\right)^{2} d t+\left(y_{0}^{\varepsilon}, \varphi^{\varepsilon}(\cdot, 0)\right)_{L^{2}(0, L)} .
$$

Taking $\varphi^{\varepsilon}=\hat{\varphi}^{\varepsilon}$ associated to $\hat{\varphi}_{T}^{\varepsilon}$ in 12 , we therefore have

$$
\left(v^{\varepsilon}, v^{\varepsilon}\right)_{L^{2}(0, T)}=\left(v^{\varepsilon}, \varepsilon \hat{\varphi}_{x}^{\varepsilon}(0, t)\right)_{L^{2}(0, T)}=-\left(y_{0}^{\varepsilon}, \hat{\varphi}^{\varepsilon}(\cdot, 0)\right)_{L^{2}(0, L)} .
$$

Consequently, if we denote by $\mathcal{A}_{\varepsilon}: L^{2}(0, L) \rightarrow L^{2}(0, L)$ the operator defined by $\mathcal{A}_{\varepsilon} y_{0}^{\varepsilon}:=-\hat{\varphi}(\cdot, 0)$, we finally may write

$$
K^{2}(\varepsilon, T, M)=\sup _{y_{0}^{\varepsilon} \in L^{2}(0, L)} \frac{\left(\mathcal{A}_{\varepsilon} y_{0}^{\varepsilon}, y_{0}^{\varepsilon}\right)_{L^{2}(0, L)}}{\left(y_{0}^{\varepsilon}, y_{0}^{\varepsilon}\right)_{L^{2}(0, L)}}
$$

and conclude that $K^{2}(\varepsilon, T, M)$ is solution of the following generalized eigenvalue problem :

$$
\sup \left\{\lambda \in \mathbb{R}: \exists y_{0}^{\varepsilon} \in L^{2}(0, L), y_{0}^{\varepsilon} \neq 0 \text {, s.t. } \mathcal{A}_{\varepsilon} y_{0}^{\varepsilon}=\lambda y_{0}^{\varepsilon} \quad \text { in } \quad L^{2}(0, L)\right\} .
$$


Remark 3 We may reformulate as well the previous extremal problem over $H_{0}^{1}(0, L)$ (seen as the dual space of $H^{-1}(0, L) \ni y(\cdot, T)$ ) in term of a generalized eigenvalue problem; we proceed as follows.

We introduce the operators $A_{\varepsilon}$ and $B_{\varepsilon}$ given by

$$
\begin{aligned}
A_{\varepsilon}: H_{0}^{1}(0, L) & \rightarrow L^{2}(0, L) \\
\varphi_{T} & \mapsto \varphi(\cdot, 0)
\end{aligned} \quad \text { and } \begin{aligned}
B_{\varepsilon}: H_{0}^{1}(0, L) & \rightarrow L^{2}(0, T) \\
\varphi_{T} & \mapsto \varepsilon \varphi_{x}(0, \cdot),
\end{aligned}
$$

where $\varphi$ solves (8). The adjoint operators $A_{\varepsilon}^{\star}$ and $B_{\varepsilon}^{\star}$ of $A_{\varepsilon}$ and $B_{\varepsilon}$ are given by :

$$
\begin{aligned}
A_{\varepsilon}^{\star}: L^{2}(0, L) & \rightarrow H^{-1}(0, L) \\
y_{0} & \mapsto y\left(T ; y_{0}, 0\right)
\end{aligned} \quad \text { and } \begin{array}{rlrl}
B_{\varepsilon}^{\star}: & L^{2}(0, L) & \rightarrow & H^{-1}(0, L) \\
v & & \mapsto y(T ; 0, v),
\end{array}
$$

where $y\left(t ; y_{0}, v\right)$ is the solution to (1) at time $t$ for the initial data $y_{0}$ and the control $v$. With these notations, we may rewrite $C_{\text {obs }}$ given by (7) as follows

$$
\begin{aligned}
C_{o b s}^{2}(\varepsilon, T, M) & =\sup _{\varphi_{T} \in H_{0}^{1}(0, L)} \frac{\left(A_{\varepsilon} \varphi_{T}, A_{\varepsilon} \varphi_{T}\right)_{L^{2}(0, L)}}{\left(B_{\varepsilon} \varphi_{T}, B_{\varepsilon} \varphi_{T}\right)_{L^{2}(0, T)}} \\
& =\sup _{\varphi_{T} \in H_{0}^{1}(0, L)} \frac{\left(\left(-\Delta^{-1}\right) A_{\varepsilon}^{\star} A_{\varepsilon} \varphi_{T}, \varphi_{T}\right)_{H_{0}^{1}(0, L)}}{\left(\left(-\Delta^{-1}\right) B_{\varepsilon}^{\star} B_{\varepsilon} \varphi_{T}, \varphi_{T}\right)_{H_{0}^{1}(0, L)}}
\end{aligned}
$$

leading to an eigenvalue problem over $H_{0}^{1}(0, L)$. Remark that the operator $B_{\varepsilon}^{\star} B_{\varepsilon}$ from $H_{0}^{1}(0, L)$ to $H^{-1}(0, L)$ associates to the initial state $\varphi_{T}$ of (8) the final state $y(\cdot, T)$ of (1) with $y_{0}=0$ and $v=\varepsilon \varphi_{x}(0, \cdot)$. $v$ is therefore the control of minimal $L^{2}(0, T)$-norm which drives the state $y$ from 0 to the trajectory $y(\cdot, T) . B_{\varepsilon}^{\star} B_{\varepsilon}$ is the so-called HUM operator.

Remark 4 Actually, the supremum of $\varphi_{T} \in H_{0}^{1}(0, L)$ in $(7)$ can be taken over $\varphi(\cdot, 0) \in L^{2}(0, L)$ (or even over $\varphi$ !) leading immediately to

$$
C_{o b s}^{2}(\varepsilon, T, M)=\sup _{\varphi(\cdot, 0) \in L^{2}(0, L)} \frac{(\varphi(\cdot, 0), \varphi(\cdot, 0))_{L^{2}(0, L)}}{\left(\mathcal{A}_{\varepsilon}^{-1} \varphi(\cdot, 0), \varphi(\cdot, 0)\right)_{L^{2}(0, L)}}
$$

in full agreement with 15 and the equality $K(\varepsilon, T, M)=C_{o b s}(\varepsilon, T, M)$.

In order to solve the eigenvalue problem (16) and get the largest eigenvalue of the operator $\mathcal{A}_{\epsilon}$, we may formally employ the power iterate method (see [11]), which reads as follows : given $y_{0}^{0} \in L^{2}(0, L)$ such that $\left\|y_{0}^{0}\right\|_{L^{2}(0, L)}=1$, compute iteratively the sequence

$$
y_{0}^{k+1}=\frac{\mathcal{A}_{\varepsilon} y_{0}^{k}}{\left\|\mathcal{A}_{\varepsilon} y_{0}^{k}\right\|_{L^{2}(0, L)}}, \quad k \geq 0 .
$$

In a finite dimensional situation, the real sequence $\left\{\left\|\mathcal{A}_{\epsilon} y_{0}^{k}\right\|_{L^{2}(0, L)}\right\}_{(k>0)}$ then converges to the eigenvalue with largest modulus of the operator $\mathcal{A}_{\varepsilon}$, so that $\sqrt{\left\|\mathcal{A}_{\varepsilon} y_{0}^{k}\right\|_{L^{2}(0, L)}} \rightarrow K(\varepsilon, T, M)$ as $k \rightarrow \infty$. The $L^{2}$-sequence $\left\{y_{0}^{k}\right\}_{(k \geq 0)}$ then converges toward the corresponding eigenvector. The first step requires to compute the image of the control operator $\mathcal{A}_{\varepsilon}$ : this is done by determining the control of minimal $L^{2}$-norm associated to the initial condition $y_{0}^{k}$.

\subsection{Approximation of the control of minimal $L^{2}$-norm}

The numerical approximation of null controls for parabolic equations is a not an easy task and has been first discussed in [8], and then in several works: we refer to the review [37. Duality theory reduces the problem to the resolution of the unconstrained extremal problem (13). In view of the regularization character of the parabolic operator, the extremal problem $\sqrt{13}$ is ill-posed as the supremum is not reached in $H_{0}^{1}(0, L)$ but in a space, say $\mathcal{H}$, defined as the completion of $H_{0}^{1}(0, L)$ for the norm $\left\|\varphi_{T}\right\|_{\mathcal{H}}:=\left\|\varepsilon \varphi_{x}(0, \cdot)\right\|_{L^{2}(0, T)}$, much larger 
than $H_{0}^{1}(0, L)$ and difficult to approximate. We refer to the review paper [37. The usual "remedy" consists to enforce the regularity $H_{0}^{1}$ and replace 13 by

$$
\left.\min _{\varphi_{T}^{\varepsilon} \in H_{0}^{1}(0, L)} J_{\beta}^{\star}\left(\varphi_{T}^{\varepsilon}\right):=\frac{1}{2} \| \varepsilon \varphi_{x}^{\varepsilon}(0, \cdot)\right)\left\|_{L^{2}(0, T)}^{2}+\left(y_{0}^{\varepsilon}, \varphi^{\varepsilon}(\cdot, 0)\right)_{L^{2}(0, L)}+\frac{\beta}{2}\right\| \varphi_{T}^{\varepsilon} \|_{H_{0}^{1}(0, L)}^{2},
$$

for any $\beta>0$ small. The resulting approximate control $v_{\beta}^{\varepsilon}=\varepsilon \varphi_{\beta, x}^{\varepsilon}(0, \cdot)$ leads to a state $y_{\beta}^{\varepsilon}$ solution of (1) satisfying the property

$$
\left\|y_{\beta}^{\varepsilon}(\cdot, T)\right\|_{H^{-1}(0, L)} \leq C \sqrt{\beta}\left\|y_{0}^{\varepsilon}\right\|_{L^{2}(0, L)}
$$

(for a constant $C>0$ independent of $\beta$ ). This penalty method is discussed in 8 ] for the boundary controllability of the heat equation (for the distributed case, we refer to [5, 19, 26]). As in [8, problem (17) may be solved using a gradient iterative method: in view of the ill-posedness of (13), such method requires an increasing number of iterates to reach convergence as $\beta$ goes to zero.

Moreover, in the context of the transport equation (1), it is necessary to chose $\beta$ in relation with the diffusion coefficient $\varepsilon$ and the controllability time $T$. Indeed, if $T>T^{\star}$, then according to Lemma 2.1, $\beta$ should be exponentially small with respect to $\varepsilon$, otherwise the minimization of $J_{\beta}^{\star}$ leads to $\varphi_{T}^{\varepsilon} \equiv 0$ and then to a null control, which is not the optimal one we expect for negative values of $M$ (in view of Theorem 2.4))! In practice, this means that as soon as $T>T^{\star}$ and $\varepsilon$ is small enough, $\beta$ is numerically equal to zero. Consequently, if $\varepsilon$ is small (as it should be!), the only case for which the Tychonoff term in $J_{\beta}^{\star}$ may be of some help is the case $T=T^{\star}$, since the corresponding $L^{2}(0, L)$-norm of the free state at time $T=T^{\star}$ is of the order $\varepsilon$.

Moreover, the occurence of boundary layers (discussed at length in the next section) both for the direct and adjoint solution $y^{\varepsilon}$ and $\varphi^{\varepsilon}$ makes the minimization of $J_{\beta}^{\star}$ even more difficult as it requires local refinement of the geometry discretization.

Theorefore, as $\varepsilon$ tends to 0 , the transport term $M y_{x}^{\varepsilon}$ makes the (numerical) approximation of the null control for 11) a challenging task. Consequently, instead of minimizing the functional $J^{\star}$ (or $J_{\beta}^{\star}$ ), we adapt [35] (devoted to the inner situation for $M=0$ and $\varepsilon=1$ ) and solve directly the corresponding optimality conditions. This leads to a space-time mixed variational formulation (following the terminology used in [35]).

\subsubsection{Mixed variational formulation}

We introduce the linear space $\Phi^{0}:=\left\{\varphi \in C^{2}\left(\overline{Q_{T}}\right), \varphi=0\right.$ on $\left.\Sigma_{T}\right\}$. For any $\eta>0$, we define the bilinear form

$$
(\varphi, \bar{\varphi})_{\Phi^{0}}:=\int_{0}^{T} \varepsilon \varphi_{x}(0, t) \varepsilon \bar{\varphi}_{x}(0, t) d t+\beta(\varphi(\cdot, T), \bar{\varphi}(\cdot, T))_{H_{0}^{1}(0, L)}+\eta \iint_{Q_{T}} L_{\varepsilon}^{\star} \varphi L_{\varepsilon}^{\star} \bar{\varphi} d x d t, \quad \forall \varphi, \bar{\varphi} \in \Phi^{0} .
$$

From the unique continuation property for the transport equation, this bilinear form defines for any $\beta \geq 0$ a scalar product. Let $\Phi_{\beta}$ be the completion of $\Phi^{0}$ for this scalar product. We denote the norm over $\Phi_{\beta}$ by $\|\cdot\|_{\Phi_{\beta}}$ such that

$$
\|\varphi\|_{\Phi_{\beta}}^{2}:=\left\|\varepsilon \varphi_{x}(0, \cdot)\right\|_{L^{2}(0, T)}^{2}+\beta\|\varphi(\cdot, T)\|_{H_{0}^{1}(0, L)}^{2}+\eta\left\|L_{\varepsilon}^{\star} \varphi\right\|_{L^{2}\left(Q_{T}\right)}^{2}, \quad \forall \varphi \in \Phi_{\beta} .
$$

Finally, we define the close subset $W_{\beta}$ of $\Phi_{\beta}$ by $W_{\beta}=\left\{\varphi \in \Phi_{\beta}: L_{\varepsilon}^{\star} \varphi=0\right.$ in $\left.L^{2}\left(Q_{T}\right)\right\}$ endowed with the same norm than $\Phi_{\beta}$. Then, for any $r \geq 0$, we define the following extremal problem :

$$
\min _{\varphi \in W_{\beta}} \hat{J}_{\beta}^{\star}(\varphi):=\frac{1}{2}\left\|\varepsilon \varphi_{x}(0, \cdot)\right\|_{L^{2}(0, T)}^{2}+\frac{\beta}{2}\|\varphi(\cdot, T)\|_{H_{0}^{1}(0, L)}^{2}+\left(y_{0}^{\varepsilon}, \varphi(\cdot, 0)\right)_{L^{2}(0, L)}+\frac{r}{2}\left\|L_{\varepsilon}^{\star} \varphi\right\|_{L^{2}\left(Q_{T}\right)}^{2} .
$$

Standard energy estimates for 11 imply that, for any $\varphi \in W_{\beta}, \varphi(\cdot, 0) \in L^{2}(0, L)$ so that the functional $\hat{J}_{\beta}^{\star}$ is well-defined over $W_{\beta}$. Moreover, since for any $\varphi \in W_{\beta}, \varphi(\cdot, T)$ belongs to $H_{0}^{1}(0, L)$, problem (20) is equivalent to the extremal problem (17). The main variable is now $\varphi$ submitted to the constraint equality (in $L^{2}\left(Q_{T}\right)$ ) $L_{\varepsilon}^{\star} \varphi=0$, which is addressed through a Lagrange multiplier, leading to a saddle-point problem.

We consider the following mixed formulation : find $\left(\varphi_{\beta}, \lambda_{\beta}\right) \in \Phi_{\beta} \times L^{2}\left(Q_{T}\right)$ solution of

$$
\left\{\begin{aligned}
a_{\beta, r}\left(\varphi_{\beta}, \bar{\varphi}\right)+b\left(\bar{\varphi}, \lambda_{\beta}\right) & =l(\bar{\varphi}), & & \forall \bar{\varphi} \in \Phi_{\beta}, \\
b\left(\varphi_{\beta}, \bar{\lambda}\right) & =0, & & \forall \bar{\lambda} \in L^{2}\left(Q_{T}\right),
\end{aligned}\right.
$$


where

$$
\begin{aligned}
& a_{\beta, r}: \Phi_{\beta} \times \Phi_{\beta} \rightarrow \mathbb{R}, \quad a_{\beta, r}(\varphi, \bar{\varphi}):=\left(\varepsilon \varphi_{x}(0, \cdot), \varepsilon \bar{\varphi}_{x}(0, \cdot)\right)_{L^{2}(0, T)}+\beta(\varphi(\cdot, T), \bar{\varphi}(\cdot, T))_{H_{0}^{1}(0, L)} \\
&+r\left(L_{\varepsilon}^{\star} \varphi, L_{\varepsilon}^{\star} \bar{\varphi}\right)_{L^{2}\left(Q_{T}\right)} \\
& b: \Phi_{\beta} \times L^{2}\left(Q_{T}\right) \rightarrow \mathbb{R}, \quad b(\varphi, \lambda):=\left(L_{\varepsilon}^{\star} \varphi, \lambda\right)_{L^{2}\left(Q_{T}\right)} \\
& l: \Phi_{\beta} \rightarrow \mathbb{R}, \quad l(\varphi):=-\left(y_{0}^{\varepsilon}, \varphi(\cdot, 0)\right)_{L^{2}(0, L)}
\end{aligned}
$$

Theorem 3.1 Assume that $\beta>0$ and $r \geq 0$.

1. The mixed formulation 21] is well-posed.

2. The unique solution $\left(\varphi_{\beta}, \lambda_{\beta}\right) \in \Phi_{\beta} \times L^{2}\left(Q_{T}\right)$ is the unique saddle-point of the Lagrangian $\mathcal{L}_{\beta, r}: \Phi_{\beta} \times L^{2}\left(Q_{T}\right) \rightarrow \mathbb{R}$ defined by

$$
\mathcal{L}_{\beta, r}(\varphi, \lambda):=\frac{1}{2} a_{\beta, r}(\varphi, \varphi)+b(\varphi, \lambda)-l(\varphi)
$$

3. The optimal function $\varphi_{\beta}$ is the minimizer of $\hat{J}_{\beta}^{\star}$ over $W_{\beta}$ while $\lambda_{\beta} \in L^{2}\left(Q_{T}\right)$ is the state of (1) in the weak sense.

Proof- The proof is very closed to the proof given in [35], Section 2.1.1. The bilinear form $a_{\beta, r}$ is continuous, symmetric and positive over $\Phi_{\beta} \times \Phi_{\beta}$. The bilinear form $b$ is continuous over $\Phi_{\beta} \times L^{2}\left(Q_{T}\right)$. Furthermore, for any $\beta>0$, the continuity of the linear form $l$ over $\Phi_{\beta}$ is deduced from the energy estimate:

$$
\|\varphi(\cdot, 0)\|_{L^{2}(0, L)}^{2} \leq C \iint_{Q_{T}}\left|L_{\varepsilon}^{\star} \varphi\right|^{2} d x d t+\|\varphi(\cdot, T)\|_{L^{2}(0, L)}^{2}, \quad \forall \varphi \in \Phi_{\beta},
$$

for some $C>0$ so that $\|\varphi(\cdot, 0)\|_{L^{2}(0, L)}^{2} \leq \max \left(C \eta^{-1}, \beta^{-1}\right)\|\varphi\|_{\Phi_{\beta}}^{2}$. Therefore, the well-posedness of the mixed formulation is a consequence of the following properties (see [7]):

- $a_{\beta, r}$ is coercive on $\mathcal{N}(b)$, where $\mathcal{N}(b)$ denotes the kernel of $b$ :

$$
\mathcal{N}(b):=\left\{\varphi \in \Phi_{\beta}: b(\varphi, \lambda)=0 \text { for every } \lambda \in L^{2}\left(Q_{T}\right)\right\} .
$$

- $b$ satisfies the usual "inf-sup" condition over $\Phi_{\beta} \times L^{2}\left(Q_{T}\right)$ : there exists $\delta>0$ such that

$$
\inf _{\lambda \in L^{2}\left(Q_{T}\right)} \sup _{\varphi \in \Phi_{\beta}} \frac{b(\varphi, \lambda)}{\|\varphi\|_{\Phi_{\beta}}\|\lambda\|_{L^{2}\left(Q_{T}\right)}} \geq \delta .
$$

The first point follows from the definition. Concerning the inf-sup condition, for any fixed $\lambda^{0} \in L^{2}\left(Q_{T}\right)$, we define the (unique) element $\varphi^{0} \operatorname{such}$ that $L^{\star} \varphi^{0}=\lambda^{0}, \varphi=0$ on $\Sigma_{T}$ and $\varphi^{0}(\cdot, T)=0$ in $L^{2}(0, L)$. The function $\varphi^{0}$ is therefore solution of the backward transport equation with source term $\lambda^{0} \in L^{2}\left(Q_{T}\right)$, null Dirichlet boundary condition and zero initial state. Moreover, since $\lambda^{0} \in L^{2}\left(Q_{T}\right)$, the following estimate proved in the Appendix A of [15] (more precisely, we refer to the inequality (94))

$$
\varepsilon\left\|\varphi_{x}^{0}(0, \cdot)\right\|_{L^{2}(0, T)} \leq C_{L, T, M}\left\|\lambda^{0}\right\|_{L^{2}\left(Q_{T}\right)},
$$

for a constant $C_{L, T, M}>0$ independent of $\varepsilon$, implies that $\varphi^{0} \in \Phi_{\beta}$. In particular, we have $b\left(\varphi^{0}, \lambda^{0}\right)=\left\|\lambda^{0}\right\|_{L^{2}}^{2}\left(Q_{T}\right)$ and

$$
\sup _{\varphi \in \Phi_{\beta}} \frac{b\left(\varphi, \lambda^{0}\right)}{\|\varphi\|_{\Phi_{\beta}}\left\|\lambda^{0}\right\|_{L^{2}\left(Q_{T}\right)}} \geq \frac{b\left(\varphi^{0}, \lambda^{0}\right)}{\left\|\varphi^{0}\right\|_{\Phi_{\beta}}\left\|\lambda^{0}\right\|_{L^{2}\left(Q_{T}\right)}}=\frac{\left\|\lambda^{0}\right\|_{L^{2}\left(Q_{T}\right)}^{2}}{\left(\left\|\varepsilon \varphi_{x}^{0}(0, \cdot)\right\|_{L^{2}(0, T)}^{2}+\eta\left\|\lambda^{0}\right\|_{L^{2}\left(Q_{T}\right)}^{2}\right)^{\frac{1}{2}}\left\|\lambda^{0}\right\|_{L^{2}\left(Q_{T}\right)}} .
$$


Combining the above two inequalities, we obtain

$$
\sup _{\varphi^{0} \in \Phi_{\beta}} \frac{b\left(\varphi^{0}, \lambda^{0}\right)}{\left\|\varphi^{0}\right\|_{\Phi_{\beta}}\left\|\lambda^{0}\right\|_{L^{2}\left(Q_{T}\right)}} \geq \frac{1}{\sqrt{C_{L, T, M}^{2}+\eta}}
$$

and, hence, 23 holds with $\delta=\left(C_{L, T, M}^{2}+\eta\right)^{-1 / 2}$.

The second point is due to the symmetry and to the positivity of the bilinear form $a_{\beta, r}$. Concerning the third point, the equality $b\left(\varphi_{\beta}, \bar{\lambda}\right)=0$ for all $\bar{\lambda} \in L^{2}\left(Q_{T}\right)$ implies that $L^{\star} \varphi_{\beta}=0$ as an $L^{2}\left(Q_{T}\right)$ function, so that if $\left(\varphi_{\beta}, \lambda_{\beta}\right) \in \Phi_{\beta} \times L^{2}\left(Q_{T}\right)$ solves the mixed formulation, then $\varphi_{\beta} \in W_{\beta}$ and $\mathcal{L}_{\beta}\left(\varphi_{\beta}, \lambda_{\beta}\right)=\hat{J}_{\beta}^{\star}\left(\varphi_{\beta}\right)$. Finally, the first equation of the mixed formulation (taking $r=0$ ) reads as follows:

$$
\int_{0}^{T} \varepsilon\left(\varphi_{\beta}\right)_{x}(0, t) \varepsilon \bar{\varphi}_{x}(0, t) d t+\beta\left(\varphi_{\beta}(\cdot, T), \bar{\varphi}(\cdot, T)\right)_{H_{0}^{1}(0, L)}-\iint_{Q_{T}} L_{\varepsilon}^{\star} \bar{\varphi} \lambda_{\beta} d x d t=l(\bar{\varphi}), \quad \forall \bar{\varphi} \in \Phi_{\beta},
$$

or equivalently, since the control is given by $v_{\beta}:=\varepsilon\left(\varphi_{\beta}\right)_{x}(0, \cdot)$,

$$
\int_{0}^{T} v_{\beta} \varepsilon \bar{\varphi}_{x}(0, t) d t+\beta\left(\varphi_{\beta}(\cdot, T), \bar{\varphi}(\cdot, T)\right)_{H_{0}^{1}(0, L)}-\iint_{Q_{T}} L_{\varepsilon}^{\star} \bar{\varphi} \lambda_{\beta} d x d t=l(\bar{\varphi}), \quad \forall \bar{\varphi} \in \Phi_{\beta} .
$$

But this means that $\lambda_{\beta} \in L^{2}\left(Q_{T}\right)$ is a solution of $(1)$ in the transposition sense. Since $y_{0} \in L^{2}(0, L)$ and $v_{\beta} \in L^{2}(0, T), \lambda_{\beta}$ coincides with the unique weak solution to (1) such that $-\Delta^{-1} \lambda_{\beta}(\cdot, T)+\beta \varphi_{\beta}(\cdot, T)=0$.

\subsubsection{Minimization with respect to the multiplier}

The augmented mixed formulation (21) allows to solve simultaneously the dual variable $\varphi_{\beta}$, argument of the conjugate functional (20), and the Lagrange multiplier $\lambda_{\beta}$, qualified as the primal variable of the problem.

Assuming that the augmentation parameter $r$ is strictly positive, we derive the corresponding extremal problem involving only that variable $\lambda_{\beta}$. For any $r>0$, let the linear operator $\mathcal{A}_{\beta, r}$ from $L^{2}\left(Q_{T}\right)$ into $L^{2}\left(Q_{T}\right)$ be defined by $\mathcal{A}_{\beta, r} \lambda:=L^{\star} \varphi$ where $\varphi=\varphi(\lambda) \in \Phi_{\beta}$ is the unique solution to

$$
a_{\beta, r}(\varphi, \bar{\varphi})=b(\bar{\varphi}, \lambda), \quad \forall \bar{\varphi} \in \Phi_{\beta} .
$$

For any $r>0$, the form $a_{\beta, r}$ defines a norm equivalent to the norm on $\Phi_{\beta}$ (see $(19)$ ), so that $(25)$ is well-posed. The following crucial lemma holds true.

LEMma 3.1 For any $r>0$, the operator $\mathcal{A}_{\beta, r}$ is a strongly elliptic, symmetric isomorphism from $L^{2}\left(Q_{T}\right)$ into $L^{2}\left(Q_{T}\right)$.

It allows to get the following proposition which permits to replace the minimization of $J_{\beta}$ over $W_{\beta}$ to the minimization of the functional $J_{\beta, r}^{\star \star}$ over $L^{2}\left(Q_{T}\right)$, which is a space much easier to approximate than $W_{\beta}$.

Proposition 3.1 For any $r>0$, let $\varphi^{0} \in \Phi_{\beta}$ be the unique solution of

$$
a_{\beta, r}\left(\varphi^{0}, \bar{\varphi}\right)=l(\bar{\varphi}), \quad \forall \bar{\varphi} \in \Phi_{\beta},
$$

and let $J_{\beta, r}^{\star \star}: L^{2}\left(Q_{T}\right) \rightarrow L^{2}\left(Q_{T}\right)$ be the functional defined by

$$
J_{\beta, r}^{\star \star}(\lambda):=\frac{1}{2}\left(\mathcal{A}_{\beta, r} \lambda, \lambda\right)_{L^{2}\left(Q_{T}\right)}-b\left(\varphi^{0}, \lambda\right) .
$$

The following equality holds :

$$
\sup _{\lambda \in L^{2}\left(Q_{T}\right)} \inf _{\varphi \in \Phi_{\beta}} \mathcal{L}_{\beta, r}(\varphi, \lambda)=-\inf _{\lambda \in L^{2}\left(Q_{T}\right)} J_{\beta, r}^{\star \star}(\lambda)+\mathcal{L}_{\beta, r}\left(\varphi^{0}, 0\right) .
$$

We refer to [35], section 2.1 for the proof in the case $M=0$.

Remark 5 By introducing appropriate weights functions (vanishing at the time $t=T$ ) leading to optimal $L^{2}$-weighted controls vanishing at time $T$, we may consider the case $\beta=0$. We refer to [35], section 2.3. 


\subsubsection{Numerical approximation}

We now turn to the discretization of the mixed formulation 21] assuming $r>0$. We follow [35] for which we refer for the details. Let then $\Phi_{\beta, h}$ and $M_{\beta, h}$ be two finite dimensional spaces parametrized by the variable $h$ such that, for any $\beta>0$,

$$
\Phi_{\beta, h} \subset \Phi_{\beta}, \quad M_{\beta, h} \subset L^{2}\left(Q_{T}\right), \quad \forall h>0 .
$$

Then, we can introduce the following approximated problems : find $\left(\varphi_{h}, \lambda_{h}\right) \in \Phi_{\beta, h} \times M_{\beta, h}$ solution of

$$
\left\{\begin{aligned}
a_{\beta, r}\left(\varphi_{h}, \bar{\varphi}_{h}\right)+b\left(\bar{\varphi}_{h}, \lambda_{h}\right) & =l\left(\bar{\varphi}_{h}\right), & & \forall \bar{\varphi}_{h} \in \Phi_{\beta, h} \\
b\left(\varphi_{h}, \bar{\lambda}_{h}\right) & =0, & & \forall \bar{\lambda}_{h} \in M_{\beta, h} .
\end{aligned}\right.
$$

The well-posedness of this mixed formulation is a consequence of two properties : the first one is the coercivity of the form $a_{\beta, r}$ on the subset $\mathcal{N}_{h}(b)=\left\{\varphi_{h} \in \Phi_{\beta, h}: b\left(\varphi_{h}, \lambda_{h}\right)=0 \quad \forall \lambda_{h} \in M_{\beta, h}\right\}$. Actually, from the relation

$$
a_{\beta, r}(\varphi, \varphi) \geq C_{r, \eta}\|\varphi\|_{\Phi_{\beta}}^{2}, \quad \forall \varphi \in \Phi_{\beta}
$$

where $C_{r, \eta}=\min \{1, r / \eta\}$, the form $a_{\beta, r}$ is coercive on the full space $\Phi_{\beta}$, and so a fortiori on $\mathcal{N}_{h}(b) \subset \Phi_{\beta, h} \subset \Phi_{\beta}$. The second property is a discrete inf-sup condition :

$$
\delta_{\beta, r, h}:=\inf _{\lambda_{h} \in M_{\beta, h}} \sup _{\varphi_{h} \in \Phi_{\beta, h}} \frac{b\left(\varphi_{h}, \lambda_{h}\right)}{\left\|\varphi_{h}\right\|_{\Phi_{\beta, h}}\left\|\lambda_{h}\right\|_{M_{\beta, h}}}>0 \quad \forall h>0 .
$$

Let us assume that this property holds. Consequently, for any fixed $h>0$, there exists a unique couple $\left(\varphi_{h}, \lambda_{h}\right)$ solution of (26). The property (27) is in general difficult to prove and strongly depends on the choice made for the approximated spaces $M_{\beta, h}$ and $\Phi_{\beta, h}$. We shall analyze numerically this property in the next section.

Remark 6 For $r=0$, the discrete formulation (26) is not well-posed over $\Phi_{\beta, h} \times M_{\beta, h}$ because the form $a_{\beta, r=0}$ is not coercive over the discrete kernel of $b$ : the equality $b\left(\lambda_{h}, \varphi_{h}\right)=0$ for all $\lambda_{h} \in M_{\beta, h}$ does not imply that $L^{\star} \varphi_{h}$ vanishes. The term $r\left\|L_{\varepsilon}^{\star} \varphi_{h}\right\|_{L^{2}\left(Q_{T}\right)}^{2}$ is a numerical stabilization term: for any $h>0$, it ensures the uniform coercivity of the form $a_{\beta, r}$ and vanishes at the limit in $h$. We also emphasize that this term is not a regularization term as it does not add any regularity to the solution $\varphi_{h}$.

The finite dimensional and conformal space $\Phi_{\beta, h}$ must be chosen such that $L^{\star} \varphi_{h}$ belongs to $L^{2}\left(Q_{T}\right)$ for any $\varphi_{h} \in \Phi_{\beta, h}$. This is guaranteed as soon as $\varphi_{h}$ possesses second-order derivatives in $L^{2}\left(Q_{T}\right)$. Any conformal approximation based on standard triangulation of $Q_{T}$ achieves this sufficient property as soon as it is generated by spaces of functions continuously differentiable with respect to the variable $x$ and spaces of continuous functions with respect to the variable $t$.

We introduce a triangulation $\mathcal{T}_{h}$ such that $\overline{Q_{T}}=\cup_{K \in \mathcal{T}_{h}} K$ and we assume that $\left\{\mathcal{T}_{h}\right\}_{h>0}$ is a regular family. Then, we introduce the space $\Phi_{\beta, h}$ as follows :

$$
\Phi_{\beta, h}=\left\{\varphi_{h} \in C^{1}\left(\overline{Q_{T}}\right):\left.\varphi_{h}\right|_{K} \in \mathbb{P}(K) \quad \forall K \in \mathcal{T}_{h}, \varphi_{h}=0 \text { on } \Sigma_{T}\right\}
$$

where $\mathbb{P}(K)$ denotes an appropriate space of polynomial functions in $x$ and $t$. In this work, we consider for $\mathbb{P}(K)$ the so-called Bogner-Fox-Schmit (BFS for short) $C^{1}$-element defined for rectangles (see [12]). In the one dimensional setting (in space), $\mathbb{P}(K)=\left(\mathbb{P}_{3, x} \otimes \mathbb{P}_{3, t}\right)(K)$ where $\mathbb{P}_{r, \xi}$ is the space of polynomial functions of order $r$ in the variable $\xi$.

We also define the finite dimensional space

$$
M_{\beta, h}=\left\{\lambda_{h} \in C^{0}\left(\overline{Q_{T}}\right):\left.\lambda_{h}\right|_{K} \in \mathbb{Q}(K) \quad \forall K \in \mathcal{T}_{h}\right\},
$$

where $\mathbb{Q}(K)$ denotes the space of affine functions both in $x$ and $t$ on the element $K$. In the one dimensional setting in space, $K$ is a rectangle and we simply have $\mathbb{Q}(K)=\left(\mathbb{P}_{1, x} \otimes \mathbb{P}_{1, t}\right)(K)$.

The resulting approximation is conformal: for any $h>0, \Phi_{\beta, h} \subset \Phi_{\beta}$ and $M_{\beta, h} \subset L^{2}\left(Q_{T}\right)$. 
Let $n_{h}=\operatorname{dim} \Phi_{\beta, h}, m_{h}=\operatorname{dim} M_{\beta, h}$ and let the real matrices $A_{\beta, r, h} \in \mathbb{R}^{n_{h}, n_{h}}, B_{h} \in \mathbb{R}^{m_{h}, n_{h}}, J_{h} \in \mathbb{R}^{m_{h}, m_{h}}$ and $L_{h} \in \mathbb{R}^{n_{h}}$ be defined by

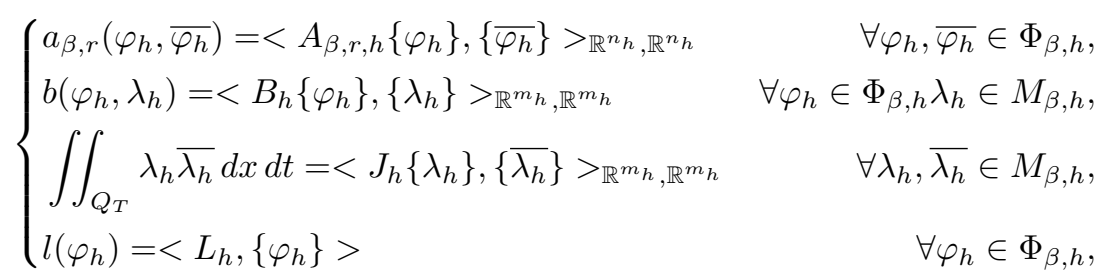

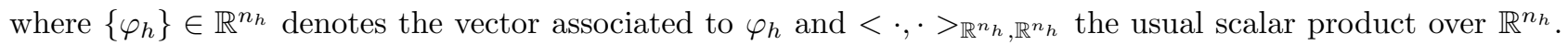
With these notations, Problem (26) reads as follows : find $\left\{\varphi_{h}\right\} \in \mathbb{R}^{n_{h}}$ and $\left\{\lambda_{h}\right\} \in \mathbb{R}^{m_{h}}$ such that

$$
\left(\begin{array}{cc}
A_{\beta, r, h} & B_{h}^{T} \\
B_{h} & 0
\end{array}\right)_{\mathbb{R}^{n_{h}+m_{h}, n_{h}+m_{h}}}\left(\begin{array}{c}
\left\{\varphi_{h}\right\} \\
\left\{\lambda_{h}\right\}
\end{array}\right)_{\mathbb{R}^{n_{h}+m_{h}}}=\left(\begin{array}{c}
L_{h} \\
0
\end{array}\right)_{\mathbb{R}^{n_{h}+m_{h}}} .
$$

\subsubsection{The discrete inf-sup test}

Before to discuss some numerical experiments, we numerically test the discrete inf-sup condition (27). Taking $\eta=r>0$ so that $a_{\beta, r}(\varphi, \bar{\varphi})=(\varphi, \bar{\varphi})_{\Phi_{\beta}}$ exactly for all $\varphi, \bar{\varphi} \in \Phi_{\beta}$, it is readily seen (see for instance [10]) that the discrete inf-sup constant satisfies

$$
\delta_{\beta, r, h}=\inf \left\{\sqrt{\delta}: B_{h} A_{\beta, r, h}^{-1} B_{h}^{T}\left\{\lambda_{h}\right\}=\delta J_{h}\left\{\lambda_{h}\right\}, \quad \forall\left\{\lambda_{h}\right\} \in \mathbb{R}^{m_{h}} \backslash\{0\}\right\} .
$$

The matrix $B_{h} A_{\beta, r, h}^{-1} B_{h}^{T}$ enjoys the same properties than the matrix $A_{\beta, r, h}$ : it is symmetric and positive definite so that the scalar $\delta_{\beta, r, h}$ defined in term of the (generalized) eigenvalue problem 290 is strictly positive. This eigenvalue problem is solved using the power iterate algorithm (assuming that the lowest eigenvalue is simple): for any $\left\{v_{h}^{0}\right\} \in \mathbb{R}^{n_{h}}$ such that $\left\|\left\{v_{h}^{0}\right\}\right\|_{2}=1$, compute for any $n \geq 0,\left\{\varphi_{h}^{n}\right\} \in \mathbb{R}^{n_{h}},\left\{\lambda_{h}^{n}\right\} \in \mathbb{R}^{m_{h}}$ and $\left\{v_{h}^{n+1}\right\} \in \mathbb{R}^{m_{h}}$ iteratively as follows :

$$
\left\{\begin{array}{l}
A_{\beta, r, h}\left\{\varphi_{h}^{n}\right\}+B_{h}^{T}\left\{\lambda_{h}^{n}\right\}=0 \\
B_{h}\left\{\varphi_{h}^{n}\right\}=-J_{h}\left\{v_{h}^{n}\right\}
\end{array} \quad, \quad\left\{v_{h}^{n+1}\right\}=\frac{\left\{\lambda_{h}^{n}\right\}}{\left\|\left\{\lambda_{h}^{n}\right\}\right\|_{2}} .\right.
$$

The scalar $\delta_{\beta, r, h}$ defined by 29 is then given by $\delta_{\beta, r, h}=\lim _{n \rightarrow \infty}\left(\left\|\left\{\lambda_{h}^{n}\right\}\right\|_{2}\right)^{-1 / 2}$.

We now reports some numerical values of $\delta_{\beta, r, h}$ with respect to $h$ for the $C^{1}$-finite element introduced in Section 3.2.3 We use the value $T=1$ and $\beta=10^{-16}$. Tables 1,2 and 3 provides the value of $\delta_{\beta, r, h}$ with respect to $h$ and $r$ for $M=1$ for $\varepsilon=10^{-1}, 10^{-2}$ and $\varepsilon=10^{-3}$, respectively. For a fixed value of the parameter $\varepsilon$, we observe as in [35], that the inf sup constant increases as $r \rightarrow 0$ and behaves like $\delta_{\beta, r, h} \approx r^{-1 / 2}$, and more importantly, is bounded by below uniformly with respect to $h$. This key property is preserved as the parameter $\varepsilon$ decreases.

\begin{tabular}{|c|ccccc|}
\hline$r$ & 10. & 1. & 0.1 & $h$ & $h^{2}$ \\
\hline$h=1 / 80$ & 0.315 & 0.919 & 1.909 & 2.359 & 2.535 \\
$h=1 / 160$ & 0.313 & 0.923 & 1.94 & 2.468 & 2.599 \\
$h=1 / 320$ & 0.313 & 0.927 & 1.969 & 2.548 & 2.658 \\
\hline
\end{tabular}

Table 1: $\delta_{\beta, r, h}$ w.r.t. $h$ and $r ; \varepsilon=10^{-1}-\beta=10^{-16}-M=1$.

The case $M=-1$ is reported in Tables 4, 5 and 6. The same behavior is observed except that we note larger values of the inf-sup constant.

Consequently, we may conclude that the finite approximation we have used "passes" the discrete inf-sup test. Such property together with the uniform coercivity of the form $a_{\beta, r}$ then imply the convergence of the approximation sequence $\left(\varphi_{h}, \lambda_{h}\right)$, unique solution of (26). As a matter of fact, the use of stabilization technics (so as to enrich the coercivity of the saddle point problem) introduced and analyzed in a closed context in 


\begin{tabular}{|c|ccccc|}
\hline$r$ & 10. & 1. & 0.1 & $h$ & $h^{2}$ \\
\hline$h=1 / 80$ & 0.311 & 0.961 & 2.423 & 3.64 & 4.473 \\
$h=1 / 160$ & 0.316 & 0.967 & 2.492 & 4.06 & 4.692 \\
$h=1 / 320$ & 0.316 & 0.971 & 2.545 & 4.406 & 4.916 \\
\hline
\end{tabular}

Table 2: $\delta_{\beta, r, h}$ w.r.t. $h$ and $r ; \varepsilon=10^{-2}-\beta=10^{-16}-M=1$.

\begin{tabular}{|c|ccccc|}
\hline$r$ & 10. & 1. & 0.1 & $h$ & $h^{2}$ \\
\hline$h=1 / 80$ & 0.310 & 0.942 & 2.121 & 3.412 & 6.012 \\
$h=1 / 160$ & 0.310 & 0.987 & 2.435 & 4.012 & 5.944 \\
$h=1 / 320$ & 0.310 & 0.969 & 2.544 & 4.561 & 5.756 \\
\hline
\end{tabular}

Table 3: $\delta_{\beta, r, h}$ w.r.t. $h$ and $r ; \varepsilon=10^{-3}-\beta=10^{-16}-M=1$.

[36, 32] is not necessary here. We emphasize that for $\beta=0$ (or $\beta \rightarrow 0$ as $h \rightarrow 0$ ), the convergence of the approximation $v_{h}$ is still an open issue. For $\beta=0$, the convergence is guarantees if a vanishing weight in time is introduced, see [19]. This however leads a priori to a different control and therefore a different definition of the cost $K(\varepsilon, T, M)$.

The choice of $r$ affects the convergence of the sequences $\varphi_{h}$ and $\lambda_{h}$ with respect to $h$ and may be very important here, in view of the sensitivity of the boundary control problem with respect to $\varepsilon$. Recall from Theorem 3.1, that for any $r \geq 0$, the multiplier $\lambda$ coincides with the controlled solution. At the finite dimensional level of the mixed formulation (26) where $r$ must be strictly positive, this property is lost for any $h$ fixed: the non zero augmentation term $r\left\|L_{\varepsilon}^{\star} \varphi_{h}\right\|_{L^{2}\left(Q_{T}\right)}$ introduces a small perturbation and requires to take $r>0$ small (in order that the approximation $\lambda_{h}$ be closed to the controlled solution $y$ ). In the sequel, the value $r=h^{2}$ is used.

\begin{tabular}{|c|ccccc|}
\hline$r$ & 10. & 1. & 0.1 & $h$ & $h^{2}$ \\
\hline$h=1 / 80$ & 0.3161 & 0.997 & 2.663 & 4.358 & 5.069 \\
$h=1 / 160$ & 0.316 & 0.9805 & 2.673 & 4.69 & 5.139 \\
$h=1 / 320$ & 0.3162 & 0.9801 & 2.653 & 4.172 & 5.171 \\
\hline
\end{tabular}

Table 4: $\delta_{\beta, r, h}$ for $\varepsilon=10^{-1}-\beta=10^{-16}-M=-1$.

\subsection{Numerical experiments}

We discuss some experiments for both $M=1$ and $M=-1$, respectively, and several values of $\varepsilon$. We consider a fixed data, independent of the parameter $\varepsilon$ : precisely, we take $y_{0}^{\varepsilon}(x)=\sin (\pi x)$ for $x \in(0, L)$ and $L=1$.

We consider regular but non uniform rectangular meshes refined near the four edges of the space-time domain $Q_{T}$. More precisely, we refine at the edge $\{x=L\} \times(0, T)$ to capture the boundary layer of length $\varepsilon$ which appear for the variable $\lambda_{h}$ when $M$ is positive (see [1]), at the edge $\{x=0\} \times(0, T)$ to approximate correctly the "control" function given by $v_{h}^{\varepsilon}:=\varepsilon \varphi_{h, x}^{\varepsilon}$, and finally at $(0, L) \times\{0, T\}$ to represent correctly the initial condition and final condition. Precisely, let $p:[0, L] \rightarrow[0, L]$ the polynomial of degree 3 such that $p(0)=0, p^{\prime}(0)=\eta_{1}, p^{\prime}(L)=\eta_{2}$ and $p(L)=L$ for some fixed $\eta_{1}, \eta_{2}>0$. The $[0, L]$ interval is therefore discretized as follows :

$$
\left\{\begin{array}{l}
{[0, L]=\cup_{j=0}^{J}\left[y_{j}, y_{j+1}\right],} \\
y_{0}=0, y_{j}-y_{j-1}=p\left(x_{j}\right)-p\left(x_{x_{j-1}}\right), \quad j=1, \cdots, J+1
\end{array}\right.
$$

where $\left\{x_{j}\right\}_{j=0, \cdots, J+1}$ is the uniform discretization of $[0, L]$ defined by $x_{j}=j h, j=0, \cdot, J+1, h=L /(J+1)$. Small values for $\eta_{1}, \eta_{2}$ lead to a refined discretization $\left\{y_{j}\right\}_{j=0, \cdots, J+1}$ at $x=0$ and $x=L$. The same procedure is used for the time discretization of $[0, T]$. In the sequel, we use $\eta_{1}=\eta_{2}=10^{-3}$. 


\begin{tabular}{|c|ccccc|}
\hline$r$ & 10. & 1. & 0.1 & $h$ & $h^{2}$ \\
\hline$h=1 / 80$ & 0.316 & 0.997 & 3.109 & 7.562 & 13.936 \\
$h=1 / 160$ & 0.3161 & 0.9997 & 3.086 & 9.433 & 14.101 \\
$h=1 / 320$ & 0.316 & 0.9809 & 3.086 & 11.1008 & 14.140 \\
\hline
\end{tabular}

Table 5: $\delta_{\beta, r, h}$ for $\varepsilon=10^{-2}-\beta=10^{-16}-M=-1$.

\begin{tabular}{|c|ccccc|}
\hline$r$ & 10. & 1. & 0.1 & $h$ & $h^{2}$ \\
\hline$h=1 / 80$ & 0.302 & 0.9129 & 2.887 & 8.16 & 39.09 \\
$h=1 / 160$ & 0.301 & 0.957 & 3.022 & 12.145 & 43.08 \\
$h=1 / 320$ & 0.301 & 0.981 & 3.084 & 16.61 & 44.29 \\
\hline
\end{tabular}

Table 6: $\delta_{\beta, r, h}$ for $\varepsilon=10^{-3}-\beta=10^{-16}-M=-1$.

Preliminary, Table 7 gives some values of the $H^{-1}$-norm of the uncontrolled solution of (1) at time $T$ associated to $y_{0}(x)=\sin (\pi x)$. We take $L=|M|=1$. A time-marching approximation scheme is used with a very fine discretization both in time and space. In agreement with Lemma 2.1. for $T$ greater than $T^{\star}$, the norm $\left\|y^{\varepsilon}(\cdot, T)\right\|_{H^{-1}(0, L)}$ decreases exponentially as $\varepsilon$ goes to zero. For $T=T^{\star}$, we observe that $\left\|y^{\varepsilon}(\cdot, T)\right\|_{H^{-1}(0, L)}=O(\varepsilon)$.

We first discuss the case $M=1$. As $\varepsilon$ goes to $0^{+}$, a boundary layer of length $\varepsilon$ appears for the approximation $\lambda_{h}^{\varepsilon}$ at $x=L$ (we refer to the next section). The profile of the solution takes along the normal the form $\left(1-e^{\frac{-M(1-x / L)}{\varepsilon}}\right)$ and is captured with a locally refined mesh. Tables 8,9 and 10 reports some numerical norms for $\varepsilon=10^{-1}, 10^{-2}$ and $10^{-3}$ respectively. These results are obtained by minimizing the functional $J_{\beta, r}^{\star \star}$ over $M_{\beta, h}$ defined in Proposition 3.1. The minimization of $J_{\beta, r}^{\star \star}$ of $M_{h}$ is performed using the conjugate gradient (CG for short in the sequel) algorithm: the stopping criterion is $\left\|g_{h}^{n}\right\|_{L^{2}\left(Q_{T}\right)} \leq 10^{-6}\left\|g_{h}^{0}\right\|_{L^{2}\left(Q_{T}\right)}$ where $g_{h}^{n}$ is the residus at the iterate $n$. The algorithm is initialized with $\lambda_{h}^{0}=0$. We refer to [35] for the details.

We take $\beta=10^{-16}$ and $r=h^{2}$ for the augmentation parameter leading to an appropriate approximation of the controlled solution $y$ by the function $\lambda_{h}$ : in particular, the optimality condition $\lambda_{h}(0, \cdot)-\varepsilon \varphi_{h, x}(0, \cdot)=0$ is well respected in $L^{2}(0, T)$. The convergence of $\sqrt{r}\left\|L_{\varepsilon}^{\star} \varphi_{h}\right\|_{L^{2}\left(Q_{T}\right)}$ (close to $\left\|L_{\varepsilon}^{\star} \varphi_{h}\right\|_{L^{2}\left(H^{-1}\right)}$ and actually sufficient to describe the solution of (1), see [13]) is also observed. As is usual, we observe a faster convergence for the norm $\left\|\lambda_{h}\right\|_{L^{2}\left(Q_{T}\right)}$ than for the norm $\left\|v_{h}\right\|_{L^{2}(0, T)}$. From $\varepsilon=10^{-1}$ to $10^{-3}$, we also clearly observe a deterioration of the convergence order with respect to $h$.

For $h=1 / 320$, Figure 2 depicts the function $\lambda_{h}(\cdot, t)$, approximation of the control $v$, for $t \in(0, T), T=1$ for $\varepsilon=10^{-1}, \varepsilon=10^{-2}$ and $\varepsilon=10^{-3}$ respectively. For large values of the diffusion coefficient $\varepsilon$, for instance $\varepsilon=$ $10^{-1}$, the transport term has a weak influence: the control of minimal $L^{2}$-norm is similar to the corresponding control for the heat equation and oscillates near the controllability time. On the contrary, for $\varepsilon$ small, typically $\varepsilon=10^{-3}$, the solution - mainly driven by the transport term - is transported along a direction closed to $(1,1 / M)=(1,1)$, so that at time $T=1 / M$, is mainly distributed in the neighborhood of $x=1$. Consequently, the control (of minimal $L^{2}$-norm) acts mainly at the beginning of the time interval, so as to have an effect, at time $T$, in the neighborhood of $x=1$. We observe a regular oscillatory and decreasing behavior of the controls.

Let us now discuss the case $M=-1$ which exhibits boundary layers for $y^{\varepsilon}$ at $x=0$ and for $\varphi^{\varepsilon}$ at $x=L$. Tables 11,12 and 13 give some numerical values with respect to $h$ for $\varepsilon=10^{-1}, 10^{-2}$ and $10^{-3}$. Concerning the behavior of the approximation with respect to $h$, similar remarks (than for $M=1$ ) can be made: the notable

\begin{tabular}{|c|ccccc|}
\hline$\varepsilon$ & $10^{-1}$ & $10^{-2}$ & $10^{-3}$ & $10^{-4}$ & $10^{-5}$ \\
\hline$T=0.9 T^{\star}$ & $2.20 \times 10^{-2}$ & $7.45 \times 10^{-4}$ & $2.76 \times 10^{-3}$ & $2.20 \times 10^{-3}$ & $2.15 \times 10^{-3}$ \\
$T=T^{\star}$ & $1.58 \times 10^{-2}$ & $2.67 \times 10^{-3}$ & $1.72 \times 10^{-4}$ & $9.76 \times 10^{-6}$ & $3.07 \times 10^{-7}$ \\
$T=1.1 T^{\star}$ & $1.12 \times 10^{-2}$ & $8.13 \times 10^{-4}$ & $1.15 \times 10^{-6}$ & $1.63 \times 10^{-19}$ & $8.62 \times 10^{-20}$ \\
\hline
\end{tabular}

Table 7: Approximation $\left\|y_{h}^{\varepsilon}(\cdot, T)\right\|_{H^{-1}(0, L)}$ w.r.t. $T$ and $\varepsilon$ for $y_{0}(x)=\sin (\pi x) . M=L=1$. 


\begin{tabular}{|c|cccc|}
\hline$h$ & $1 / 80$ & $1 / 160$ & $1 / 320$ & $1 / 640$ \\
\hline$\sqrt{r}\left\|L_{\varepsilon}^{\star} \varphi_{h}^{\varepsilon}\right\|_{L^{2}\left(Q_{T}\right)}$ & $7.76 \times 10^{-2}$ & $3.01 \times 10^{-2}$ & $1.12 \times 10^{-2}$ & $7.12 \times 10^{-3}$ \\
$\frac{\left\|\varepsilon \varphi_{x}^{\varepsilon}(0, \cdot)-\lambda_{h}^{\varepsilon}(0, \cdot)\right\|_{L^{2}(0, T)}}{\left\|\lambda_{h}(0, \cdot)\right\|_{L^{2}(0, T)}}$ & $1.06 \times 10^{-2}$ & $4.45 \times 10^{-3}$ & $1.97 \times 10^{-3}$ & $7.61 \times 10^{-4}$ \\
$\left\|v_{h}^{\varepsilon}\right\|_{L^{2}(0, T)}$ & 0.324 & 0.357 & 0.3877 & 0.3912 \\
$\left\|\lambda_{h}^{\varepsilon}\right\|_{L^{2}\left(Q_{T}\right)}$ & 0.367 & 0.366 & 0.362 & 0.363 \\
$\left\|\lambda_{h}^{\varepsilon}(\cdot, T)\right\|_{H^{-1}(0, L)}$ & $4.47 \times 10^{-6}$ & $9.59 \times 10^{-7}$ & $2.03 \times 10^{-7}$ & $1.01 \times 10^{-7}$ \\
$\sharp \mathrm{CG}$ iterate & 76 & 117 & 175 & 231 \\
\hline
\end{tabular}

Table 8: Mixed formulation 21 - $r=h^{2} ; \varepsilon=10^{-1} ; \beta=10^{-16}-M=L=1$.

\begin{tabular}{|c|cccc|}
\hline$h$ & $1 / 80$ & $1 / 160$ & $1 / 320$ & $1 / 640$ \\
\hline$\sqrt{r}\left\|L_{\varepsilon}^{\star} \varphi_{h}^{\varepsilon}\right\|_{L^{2}\left(Q_{T}\right)}$ & $5.86 \times 10^{-1}$ & $2.43 \times 10^{-1}$ & $1.41 \times 10^{-1}$ & $9.12 \times 10^{-2}$ \\
$\frac{\left\|\varepsilon \varphi_{x}^{\varepsilon}(0, \cdot)-\lambda_{h}^{\varepsilon}(0, \cdot)\right\|_{L^{2}(0, T)}}{\left\|\lambda_{h}^{\varepsilon}(0, \cdot)\right\|_{L^{2}(0, T)}}$ & $2.5 \times 10^{-2}$ & $1.24 \times 10^{-2}$ & $6.04 \times 10^{-3}$ & $2.89 \times 10^{-3}$ \\
$\left\|v_{h}^{\varepsilon}\right\|_{L^{2}(0, T)}$ & 1.391 & 2.392 & 2.929 & 3.316 \\
$\left\|\lambda_{h}^{\varepsilon}\right\|_{L^{2}\left(Q_{T}\right)}$ & 0.518 & 0.6001 & 0.789 & 0.832 \\
$\left\|\lambda_{h}^{\varepsilon}(\cdot, T)\right\|_{H^{-1}(0, L)}$ & $5.46 \times 10^{-6}$ & $3.56 \times 10^{-6}$ & $8.77 \times 10^{-7}$ & $6.12 \times 10^{-8}$ \\
$\sharp \mathrm{CG}$ iterate & 53 & 93 & 155 & 181 \\
\hline
\end{tabular}

Table 9: Mixed formulation 21 - $r=h^{2} ; \varepsilon=10^{-2} ; \beta=10^{-16}-M=L=1$.

\begin{tabular}{|c|cccc|}
\hline$h$ & $1 / 80$ & $1 / 160$ & $1 / 320$ & $1 / 640$ \\
\hline$\sqrt{r}\left\|L_{\varepsilon}^{\star} \varphi_{h}^{\varepsilon}\right\|_{L^{2}\left(Q_{T}\right)}$ & $1.75 \times 10^{-1}$ & $1.01 \times 10^{-1}$ & $8.51 \times 10^{-2}$ & $6.91 \times 10^{-2}$ \\
$\frac{\left\|\varepsilon \varphi_{x}^{\varepsilon}(0, \cdot)-\lambda_{h}^{\varepsilon}(0, \cdot)\right\|_{L^{2}(0, T)}}{\left\|\lambda_{h}^{\varepsilon}(0, \cdot)\right\|_{L^{2}(0, T)}}$ & $4.87 \times 10^{-2}$ & $2.43 \times 10^{-2}$ & $1.3 \times 10^{-4}$ & $7.19 \times 10^{-5}$ \\
$\left\|v_{h}^{\varepsilon}\right\|_{L^{2}(0, T)}$ & 0.231 & 0.713 & 0.855 & 0.911 \\
$\left\|\lambda_{h}^{\varepsilon}\right\|_{L^{2}\left(Q_{T}\right)}$ & 0.498 & 0.5015 & 0.5210 & 0.5319 \\
$\left\|\lambda_{h}^{\varepsilon}(\cdot, T)\right\|_{H^{-1}(0, L)}$ & $1.17 \times 10^{-6}$ & $3.69 \times 10^{-7}$ & $1.20 \times 10^{-7}$ & $8.12 \times 10^{-8}$ \\
$\sharp$ CG iterate & 29 & 68 & 129 & 151 \\
\hline
\end{tabular}

Table 10: Mixed formulation 21 $-r=h^{2} ; \varepsilon=10^{-3} ; \beta=10^{-16}-M=L=1$.
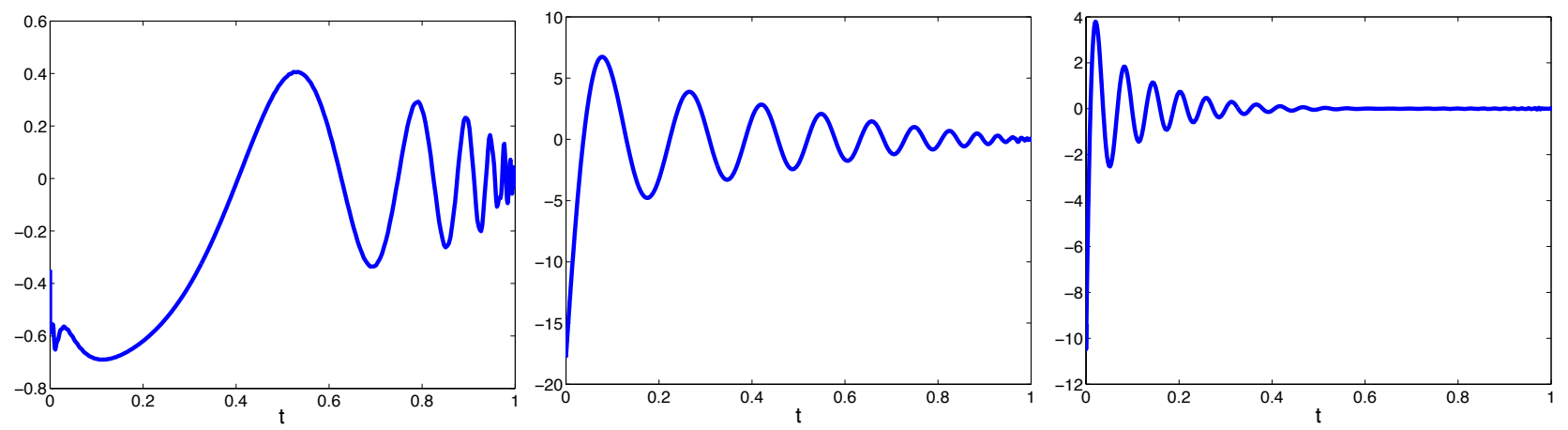

Figure 2: Approximation $\lambda_{h}^{\varepsilon}(0, t)$ of the control w.r.t. $t \in[0, T]$ for $\varepsilon=10^{-1}, 10^{-2}$ and $10^{-3} ; T=L=M=1$; $r=h^{2}-h=1 / 320$. 


\begin{tabular}{|c|cccc|}
\hline$h$ & $1 / 80$ & $1 / 160$ & $1 / 320$ & $1 / 640$ \\
\hline$\sqrt{r}\left\|L_{\varepsilon}^{\star} \varphi_{h}^{\varepsilon}\right\|_{L^{2}\left(Q_{T}\right)}$ & 1.51 & 0.731 & 0.231 & 0.101 \\
$\left\|\varepsilon \varphi_{x}^{\varepsilon}(0, \cdot)-\lambda_{h}^{\varepsilon}(0, \cdot)\right\|_{L^{2}(0, T)}$ & $9.19 \times 10^{-3}$ & $3.87 \times 10^{-3}$ & $1.61 \times 10^{-3}$ & $1.12 \times 10^{-3}$ \\
$\left\|\lambda_{h}^{\varepsilon}(0, \cdot)\right\|_{L^{2}(0, T)}$ & 28.16 & 39.26 & 49.96 & 52.03 \\
$\left\|v_{h}^{\varepsilon}\right\|_{L^{2}(0, T)}$ & 5.74 & 7.96 & 9.05 & 10.12 \\
$\left\|\lambda_{h}^{\varepsilon}\right\|_{L^{2}\left(Q_{T}\right)}$ & $8.35 \times 10^{-4}$ & $1.82 \times 10^{-4}$ & $3.97 \times 10^{-5}$ & $1.12 \times 10^{-5}$ \\
$\left\|\lambda_{h}^{\varepsilon}(\cdot, T)\right\|_{H^{-1}(0, L)}$ & 48 & 80 & 129 & 157 \\
$\sharp$ CG iterate & & & & \\
\hline
\end{tabular}

Table 11: Mixed formulation 21 $-r=h^{2} ; \varepsilon=10^{-1} ; \beta=10^{-16}-M=-1$.

\begin{tabular}{|c|cccc|}
\hline$h$ & $1 / 80$ & $1 / 160$ & $1 / 320$ & $1 / 640$ \\
\hline$\sqrt{r}\left\|L_{\varepsilon}^{\star} \varphi_{h}^{\varepsilon}\right\|_{L^{2}\left(Q_{T}\right)}$ & 5.291 & 2.134 & 1.213 & 0.591 \\
$\left\|\varepsilon \varphi_{x}^{\varepsilon}(0, \cdot)-\lambda_{h}^{\varepsilon}(0, \cdot)\right\|_{L^{2}(0, T)}$ & $5.27 \times 10^{-4}$ & $2.08 \times 10^{-2}$ & $8.05 \times 10^{-3}$ & $5.01 \times 10^{-3}$ \\
$\left\|\lambda_{h}^{\varepsilon}(0, \cdot)\right\|_{L^{2}(0, T)}$ & 250.54 & 457.78 & 666.902 & 712.121 \\
$\left\|v_{h}^{\varepsilon}\right\|_{L^{2}(0, T)}$ & 6.76 & 10.05 & 13.111 & 15.301 \\
$\left\|\lambda_{h}^{\varepsilon}\right\|_{L^{2}\left(Q_{T}\right)}$ & $1.54 \times 10^{-3}$ & $2.08 \times 10^{-3}$ & $1.71 \times 10^{-3}$ & $6.12 \times 10^{-4}$ \\
$\left\|\lambda_{h}^{\varepsilon}(\cdot, T)\right\|_{H^{-1}(0, L)}$ & 22 & 41 & 79 & 101 \\
$\sharp$ CG iterate & & & & \\
\hline
\end{tabular}

Table 12: Mixed formulation 21 $-r=h^{2} ; \varepsilon=10^{-2} ; \beta=10^{-16}-M=-1$.

difference is a lower rate of convergence, probably due to the singularity of the controls we obtain. Precisely, for the same data as in the case $M=1$, Figure 3 depicts the "control" function $\lambda_{h}^{\varepsilon}(0, t)$ for $t \in(0, T), T=1$ for $\varepsilon=10^{-1}, \varepsilon=10^{-2}$ and $\varepsilon=10^{-3}$ respectively. The behavior of the control is quite different from the previous case. For $\varepsilon$ large, typically $\varepsilon=10^{-1}$, the control is again similar to the control we observe for the heat equation, with an oscillatory behavior at the final time. We observe however that the corresponding norm is significantly larger that for the case $M=1$ : this is due to the fact, that for $M<0$, the transport term "pushes" the solution toward $x=0$ where the control acts: this reduces the effect of the control which therefore must be stronger. For $\varepsilon$ small, the solution is mainly transported along the direction $(1,1 / M)=(1,-1)$ so that at time $T$, the solution is mainly concentrated in the neighborhood of $x=0$. For this reason, the control mainly acts at the end of the time interval: any action of the control not concentrated at the end of the time interval would be useless because pushed back to the edge $x=0$ and will produce a larger $L^{2}$-norm. As $\varepsilon$ goes to zero, the control is getting concentrated at the terminal time with an oscillatory behavior and large amplitudes. This fact may explain why the behavior of the cost of control with respect to $\varepsilon$ observed in [15, 21, 29] is singular for negatives values of $M$. For $M>0$, the transport term "helps" the control to act on the edge $x=1$ while for $M<0$, the transport term is against the control and reduces its action. For this reason, the numerical approximation of controls for $M=-1$ is definitively more involved and requires to take a very fine discretization.

We also observe, both for $M=1$ and $M=-1$, that from $\varepsilon=10^{-2}$ to $\varepsilon=10^{-3}$, the $L^{2}$-norm $\left\|v^{\varepsilon}\right\|_{L^{2}(0, T)}$ decreases. Probably, as $\varepsilon$ goes to zero, this norm goes to zero. This does not contradict Theorem 2.2 obtained for the dependent of $\varepsilon$ initial condition given by (11).

\begin{tabular}{|c|cccc|}
\hline$h$ & $1 / 80$ & $1 / 160$ & $1 / 320$ & $1 / 640$ \\
\hline$\sqrt{r}\left\|L_{\varepsilon}^{\star} \varphi_{h}^{\varepsilon}\right\|_{L^{2}\left(Q_{T}\right)}$ & 7.12 & 2.14 & 1.31 & 0.59 \\
$\left\|\varepsilon \varphi_{x}^{\varepsilon}(0, \cdot)-\lambda_{h}^{\varepsilon}(0 \cdot \cdot)\right\|_{L^{2}(0, T)}$ & $2.87 \times 10^{-1}$ & $7.76 \times 10^{-2}$ & $4.31 \times 10^{-2}$ & $2.12 \times 10^{-2}$ \\
\hline$\left\|\lambda_{h}^{\varepsilon}(0, \cdot)\right\|_{L^{2}(0, T)}$ & $0.281 \times 10^{-1}$ & 2.35 & 18.98 & 21.23 \\
$\left\|v_{h}^{\varepsilon}\right\|_{L^{2}(0, T)}$ & $4.97 \times 10^{-1}$ & $5.01 \times 10^{-1}$ & $6.38 \times 10^{-1}$ & $7.23 \times 10^{-1}$ \\
$\left\|\lambda_{h}^{\varepsilon}\right\|_{L^{2}\left(Q_{T}\right)}$ & $2.03 \times 10^{-5}$ & $3.28 \times 10^{-5}$ & $6.01 \times 10^{-5}$ & $8.01 \times 10^{-5}$ \\
$\left\|\lambda_{h}^{\varepsilon}(\cdot, T)\right\|_{H^{-1}(0, L)}$ & 7 & 11 & 23 & 26 \\
$\sharp$ CG iterate & 7 & & &
\end{tabular}

Table 13: Mixed formulation 21 $-r=h^{2} ; \varepsilon=10^{-3} ; \beta=10^{-16}-M=-1$. 

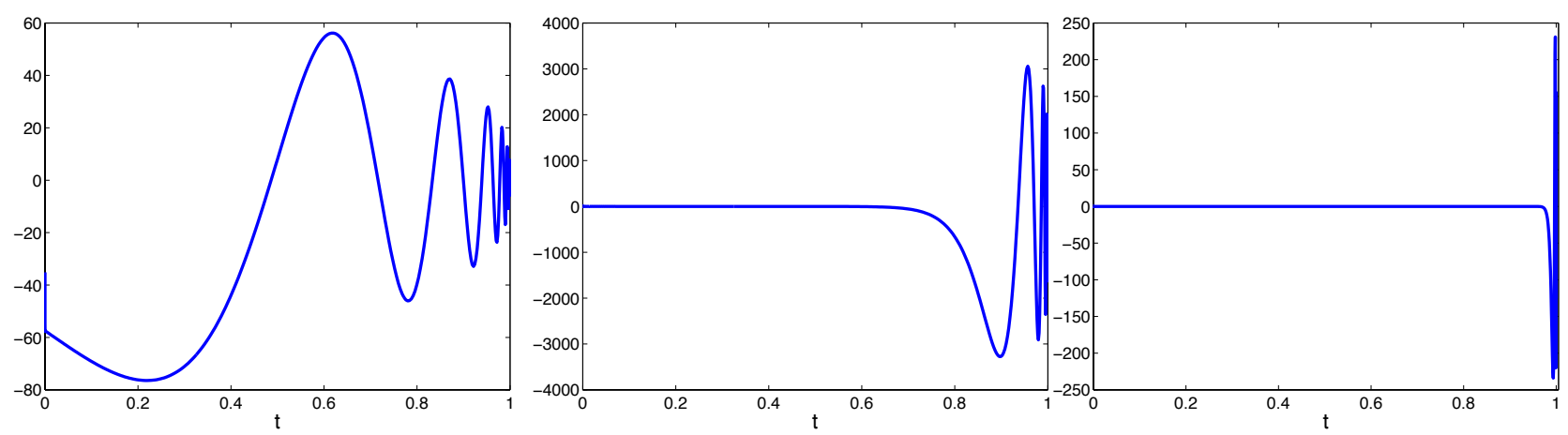

Figure 3: Approximation $\lambda_{h}^{\varepsilon}(0, t)$ of the control w.r.t. $t \in[0, T]$ for $\varepsilon=10^{-1}, 10^{-2}$ and $10^{-3} ; T=L=-M=1$; $r=h^{2}-h=1 / 320$.

\subsection{Numerical approximation of the cost of control}

We now turn to the numerical approximation of the cost of control $K(\varepsilon, T, M)$ defined by (3) and address numerically the resolution of the generalized eigenvalue problem (16). Let $V_{h}$ be a conformal approximation of the space $L^{2}(0, L)$ for all $h>0$. At the discrete level, problem 16 leads to the following finite dimensional eigenvalue problem:

$$
\sup \left\{\lambda \in \mathbb{R}: \exists y_{0, h} \in V_{h}, y_{0, h} \neq 0 \text {, s.t. } \mathcal{A}_{\varepsilon} y_{0, h}=\lambda y_{0, h} \quad \text { in } \quad V_{h}\right\} .
$$

$\mathcal{A}_{\varepsilon} y_{0, h}$ in $L^{2}(0, L)$ is defined as $-\varphi_{h}(\cdot, 0)$ where $\varphi_{h} \in \Phi_{\beta, h}$ solves the variational formulation 26). Consequently, from the definition of $\Phi_{\beta, h}$ in 28 , the space $V_{h}$ is the set of $C^{1}$-functions and piecewise polynomial of order 3 :

$$
V_{h}=\left\{y_{0, h} \in C^{1}([0, L]):\left.y_{0, h}\right|_{K} \in \mathbb{P}_{3, x} \quad \forall K \in T_{h}\right\}
$$

where $T_{h}$ is the triangulation of $[0, L]$ defined by $(30)$. This kind of finite dimensional eigenvalue problems may be solved using the power iterate method (see [11]): the algorithm is as follows: given $y_{0, h}^{0} \in L^{2}(0, L)$ such that $\left\|y_{0, h}^{0}\right\|_{L^{2}(0, L)}=1$, compute for all $k \geq 0$,

$$
y_{0, h}^{k+1}=\frac{\mathcal{A}_{\varepsilon} y_{0, h}^{k}}{\left\|\mathcal{A}_{\varepsilon} y_{0, h}^{k}\right\|_{L^{2}(0, L)}}, \quad k \geq 0 .
$$

For any fixe $h>0$, the real sequence $\left\{\left\|z_{0, h}^{k}\right\|_{L^{2}(0, L)}\right\}$ then converges to the eigenvalue with largest modulus of the operator $\mathcal{A}_{\varepsilon}$ restricted to eigenvectors in $V_{h}$, so that $\sqrt{\left\|\mathcal{A}_{\varepsilon} y_{0, h}^{k}\right\|_{L^{2}(0,1)}} \rightarrow K_{h}(\varepsilon, T, M, L)$ as $k \rightarrow \infty$. The sequence $\left\{y_{0, h}^{k}\right\}_{k>0}$ converges to the corresponding eigenvector. The first step requires to compute the image of the control operator $\mathcal{A}_{\varepsilon}$ : this is done by solving the mixed formulation 26 taking $y_{0, h}^{k}$ as initial condition for 11. The algorithm is stopped as soon as the sequence $\left\{\mathcal{A}_{\varepsilon} y_{0, h}^{k}\right\}_{k \geq 0}$ satisfies

$$
\left|\left\|\mathcal{A}_{\varepsilon} y_{0, h}^{k}\right\|_{L^{2}(0, L)}-\left\|\mathcal{A}_{\varepsilon} y_{0, h}^{k-1}\right\|_{L^{2}(0, L)}\right| \leq 10^{-3}\left\|\mathcal{A}_{\varepsilon} y_{0, h}^{k-1}\right\|_{L^{2}(0, L)},
$$

for some $k>0$.

We now report the numerical values for $L=1$ and $M= \pm 1$. We initialize the algorithm with

$$
y_{0}^{0}(x)=\frac{e^{-\frac{M x}{2 \epsilon}} \sin (\pi x)}{\left\|e^{-\frac{M x}{2 \epsilon}} \sin (\pi x)\right\|_{L^{2}(0, L)}}, \quad x \in(0, L)
$$



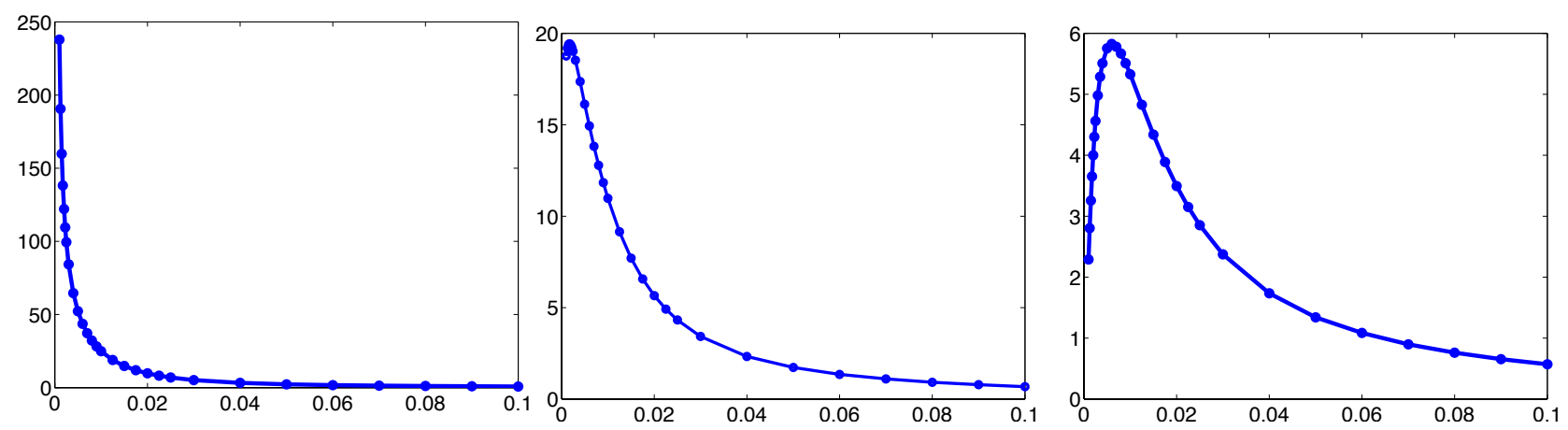

Figure 4: Cost of control $K_{h}(\varepsilon, T, M)$ w.r.t. $\varepsilon \in\left[10^{-3}, 10^{-1}\right]$ for $T=0.95 T^{\star}, T=T^{\star}$ and $T=1.05 T^{\star}$; $L=M=1 ; r=h^{2}-h=1 / 320$.

\subsubsection{Cost of control in the case $M=1$}

The tables in the annexe section report on the approximations obtained of the cost of control $K_{h}(\varepsilon, T, M)$ for $M=1$ with respect to $T$ and $\varepsilon$. They corresponds to the discretization $h=1 / 320$. As expected, for $T$ strictly lower than $T^{\star}=1$, here $T=0.95$ and $T=0.99$, we obtain that the $\operatorname{cost} K_{h}(\varepsilon, T, M)$ blows up as $\varepsilon$ goes to zero. This is in agreement with the fact, that for $T<T^{\star}$, the system (1) is not uniformly controllable with respect to the initial data $y_{0}^{\varepsilon}$ and $\varepsilon$.

Figure 4-Left displays the approximations with respect to $\varepsilon$ for $T=0.95 T^{\star}$. On the other hand, for $T$ larger than $T^{\star}=1$, we observe that the numerical approximation of $K(\varepsilon, T, M)$ is bounded with respect to $\varepsilon$. More precisely, the cost is not monotonous with respect to $\varepsilon$ as it reaches a maximal value for $\varepsilon \approx 1.75 \times 10^{-3}$ for $T=T^{\star}$ and $\varepsilon \approx 6 \times 10^{-3}$ for $T=1.05 T^{\star}$ (see Figure 4 ).

Figure 5-Left displays the approximation of the initial data $y_{0}^{\varepsilon} \in L^{2}(0, L)$ solution of the optimal problem 15) for $T=1$ and $\varepsilon=10^{-1}, 10^{-2}$ and $10^{-3}$. As $\varepsilon$ decreases, the optimal initial condition $y_{0}^{\varepsilon}$ with $\left\|y_{0}^{\varepsilon}\right\|_{L^{2}(0, L)}=1$ gets concentrated at $x=0$. Again, this is in agreement with the intuition since such condition produces (in the uncontrolled situation) larger values of $\left\|y^{\varepsilon}(\cdot, T)\right\|_{H^{-1}(\Omega)}$. It should be noted however that the solutions we get are different from $e^{-\frac{M x}{2 \epsilon}} \sin (\pi x) /\left\|e^{-\frac{M x}{2 \epsilon}} \sin (\pi x)\right\|_{L^{2}(0, L)}$. Moreover, they are apparently independent of the controllability time $T$ (at least for the values of $T$ close to $1 / M$ we have used). Remark also that the initial data $y_{0}(x)=e^{\frac{M x}{2 \epsilon}} \sin (\pi x) /\left\|e^{\frac{M x}{2 \epsilon}} \sin (\pi x)\right\|_{L^{2}(0, L)}$ highlighted in [15, 30] leads to a much lower numerical value of $\left\|v_{h}^{\varepsilon}\right\|_{L^{2}(0, L)}$.

For each values of $\varepsilon$ and $T$, the convergence of the power iterate algorithm is fast: the stopping criterion (31) is reached in less than 5 iterates.

Remark 7 We also emphasize that the space-time variational approximation we use is very well-suited to the mesh adaptivity. Figure 6 depicts the non uniform time-space mesh obtained after 5 adaptations starting from a regular mesh, corresponding to $\varepsilon=10^{-3}$ and $M=1$. A refinement appears at $x=L$, along the characteristic $L x-M t=0$, at the beginning of the time interval for $x=0$ where the control is mainly active and oscillating and also in the neighborhood of the corner $(x, t)=(L, T)$ where the adjoint solution is oscillating as well.

Remark 8 In order to avoid the use of $C^{1}$-approximation in space, one may preliminary rewrite the adjoint equation into a first order system

$$
L_{\varepsilon, 1}^{\star}(\varphi, p):=\varphi_{t}+p_{x}+M \varphi_{x}=0, \quad L_{\varepsilon, 2}^{\star}(\varphi, p):=p-\varepsilon \varphi_{x}=0, \quad \text { in } \quad Q_{T},
$$

involving the flux $p$ and for which a generalized observability inequality holds true as well. As discussed in [36] and [34] for the case of the heat and wave equations respectively, this allows to use simply $H^{1}\left(Q_{T}\right)$ approximation. On the other hand, the corresponding discrete inf-sup constant is generally not uniformly bounded by below; 

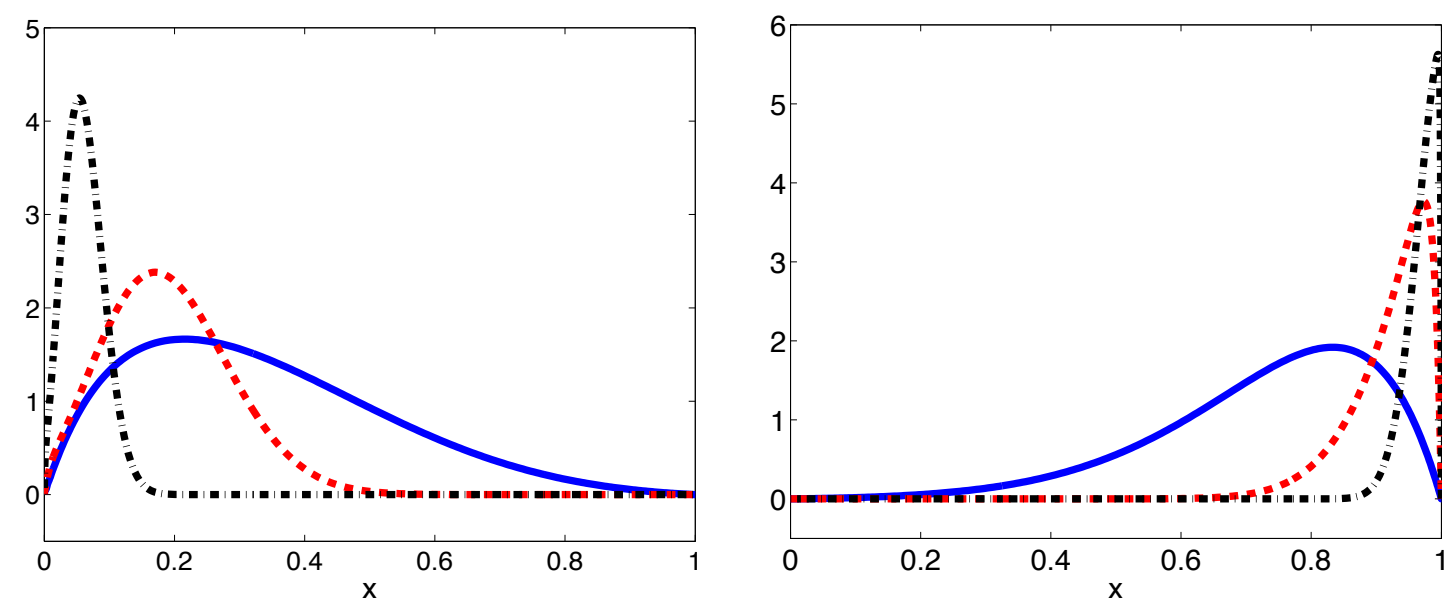

Figure 5: The optimal initial condition $y_{0}^{\varepsilon}$ in $(0, L)$ for $\varepsilon=10^{-1}$ (full line), $\varepsilon=10^{-2}$ (dashed line) and $\varepsilon=10^{-3}$ (dashed-dotted line) and $T=L=1 ; r=h^{2}-h=1 / 320 . M=1$ (Left) and $M=-1$ (right).

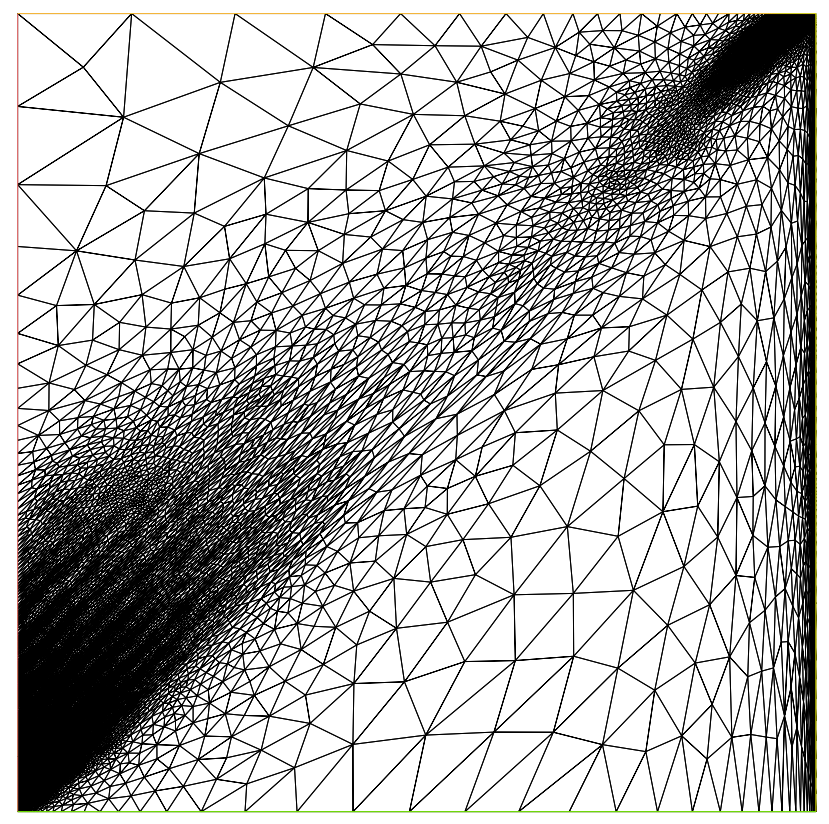

Figure 6: $L=M=1 ; \varepsilon=10^{-3}$; Non uniform adapted mesh over the space time domain $Q_{T}$. 


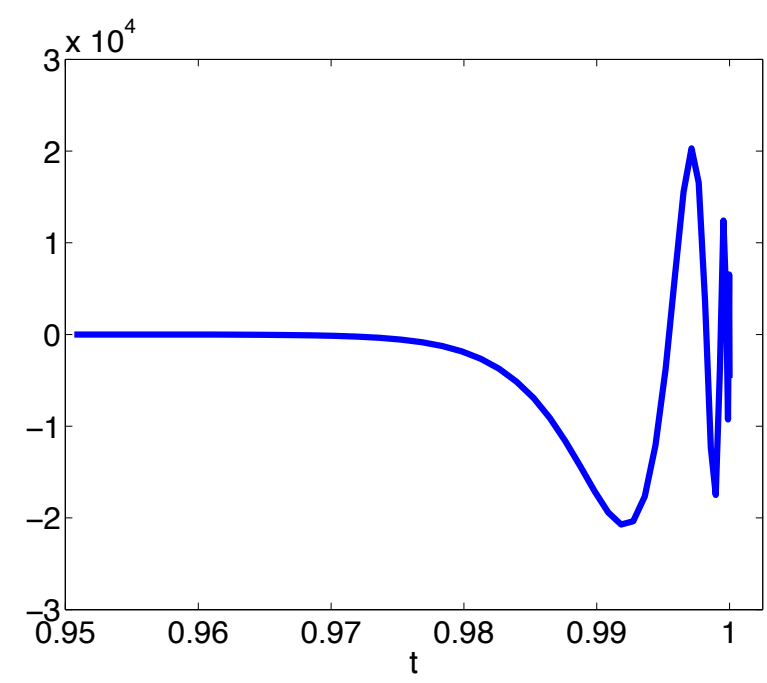

Figure 7: Approximation $\lambda_{h}^{\varepsilon}(0, t)$ of the corresponding control w.r.t. $t \in[0, T]$ for $\varepsilon=10^{-3}$ and $T=L=-M=$ $1 ; r=h^{2}-h=1 / 320$.

this requires the use of stabilized technics à la Barbosa-Hugues [3], leading here to the following stabilizedaugmentation formulation

$$
\left\{\begin{aligned}
\sup _{\left(\lambda_{1}, \lambda_{2}\right) \in \Lambda} \inf _{(\varphi, p) \in \Phi_{\beta}} & \mathcal{L}_{r, \alpha}\left((\varphi, p),\left(\lambda_{1}, \lambda_{2}\right)\right):=\frac{1}{2}\|p(0, \cdot)\|_{L^{2}(0, T)}^{2}+\left(y_{0}^{\varepsilon}, \varphi(0, \cdot)\right)_{L^{2}(0, L)} \\
+ & <\lambda_{1}, L_{\varepsilon, 1}^{\star} \varphi>_{L^{2}\left(Q_{T}\right)}+<\lambda_{2}, L_{\varepsilon, 2}^{\star} \varphi>_{L^{2}\left(Q_{T}\right)} \\
& +\frac{r_{1}}{2}\left\|L_{\varepsilon, 1}^{\star}(\varphi, p)\right\|_{L^{2}\left(Q_{T}\right)}^{2}+\frac{r_{2}}{2}\left\|L_{\varepsilon, 2}^{\star}(\varphi, p)\right\|_{L^{2}\left(Q_{T}\right)}^{2} \\
& -\frac{\alpha_{1}}{2}\left\|L_{\varepsilon, 1}\left(\lambda_{1}, \lambda_{2}\right)\right\|_{L^{2}\left(Q_{T}\right)}^{2}-\frac{\alpha_{2}}{2}\left\|L_{\varepsilon, 2}\left(\lambda_{1}, \lambda_{2}\right)\right\|_{L^{2}\left(Q_{T}\right)}^{2}
\end{aligned}\right.
$$

with $r_{1}, r_{2}>0$ (augmentation parameters) and $\alpha_{1}, \alpha_{2}$ (stabilization terms). The annexe provides the implementation of the corresponding mixed formulation using $\mathbb{P}^{1}$-elements associated to the FreeFem ++ package developed at the University Paris 6 (see [23]), which is very well adapted to our space-time setting.

\subsubsection{Cost of control in the case $M=-1$}

Tables in the annexe section reports the approximation of the cost of control $K(\varepsilon, T, M)$ for $M=-1$ and $T=T^{\star}$ with respect to $\varepsilon \in\left[10^{-3}, 10^{-1}\right]$. With respect to the positive case, the notable difference is the amplitude of the cost, as expected much larger, since the transport term now acts "against" the control. For instance, for $\varepsilon=10^{-3}$, we obtain $K(\varepsilon, T, M) \approx 18.75$ for $M=1$ and $K_{h}(\varepsilon, T, M) \approx 1.07 \times 10^{4}$ for $M=-1$. Moreover, the corresponding optimal initial condition $y_{0}$ is supported as $\varepsilon \rightarrow 0$ at the right extremity $x=1$ (see figure 5-Right) leading to a corresponding control localized at $t=T$, with very large amplitude and oscillations, as shown on Figure 7 for $\varepsilon=10^{-3}$. Such oscillations are difficult to capture numerically and are very sensitive to the discretization used. On the other hand, we observe, as for $M=1$, that the $\operatorname{cost} K_{h}(\varepsilon, T, M)$ does not blow up as $\varepsilon \rightarrow 0$, in contradiction with the theoretical results from [15, 30]. The discretization used is not fine enough here to capture the highly oscillatory behavior of the control near the controllability time $T$ (in contrast to the positive case) and very likely leads to an uncorrect approximation of the controls. For $T$ lower than $1 /|M|$, as expected, we observe that the cost blows up, while for $T$ strictly greater than $1 /|M|$, the cost decreases to zero with $\varepsilon$. 


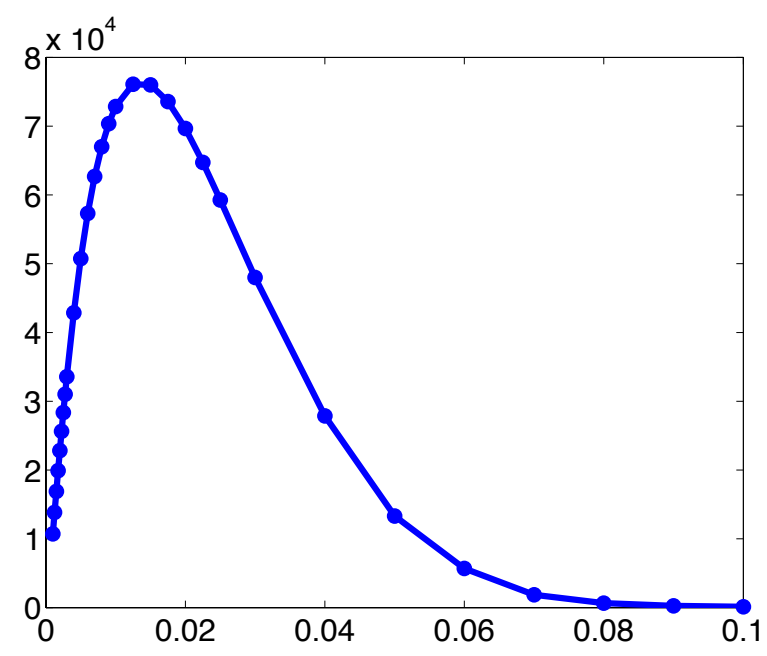

Figure 8: Cost of control $K(\varepsilon, T, M)$ w.r.t. $\varepsilon \in\left[10^{-3}, 10^{-1}\right]$ for $T=L / M$ and $L=-M=1 ; r=h^{2}-h=1 / 320$.

\subsection{Conclusion of the numerical study}

For $M>0$, the "worst" initial data we observe are concentrated at $x=0$ leading to a control distributed at the beginning of the time interval, and vanishing as $t \rightarrow T$. In this case, controls $v$ are smooth and easily approximated. Vanishing exponentially weights as considered in [35] leading to strong convergent results (w.r.t. h) are therefore not necessary here. Consequently, for $M>0$, we are confident with the numerical approximation obtained and may conjecture that the minimal time of uniform controllability w.r.t. $\varepsilon$ is $T_{M}=T^{\star}$. The situation is much more singular for $M<0$ for which the transport term acts "against" the control. The optimal initial data are concentrated as the right extremity leading to a highly singular controls at the end of the time interval. Such controls, similar to the controls we observe for the heat equation (see [37) are difficult to approximate. The strong convergent approximation of controls w.r.t. $h$ is still open in such situation. In both situations, we also suspect, that at limit in $\varepsilon$, the worst initial condition is related to the function $e^{-\frac{M x}{2 \varepsilon}} \sin \left(\frac{\pi x}{L}\right), x \in(0, L)$.

We also emphasize that the numerical approach is restricted to large enough values of $\varepsilon$, since for small $\varepsilon$, the numerical approximation can not distinguish, in view of the lemma 2.1, approximate and null controls. Let us however comment possible perspectives to improve the resolution of this singular controllability problem.

a) A way to recover a strong convergent approximation with respect to $h$ is to force the control to vanish exponentially at time $T$ of the form $v(t):=\varepsilon \rho^{-2}(t) \varphi_{x}(0, t)$, with $\rho(t):=O\left(e^{1 /(T-t)}\right)$. Remark that this modifies the cost of control as follows:

$$
K_{\rho}(\varepsilon, T, M):=\sup _{\left\|y_{0}^{\varepsilon}\right\|_{L^{2}(0, L)}=1}\left\{\min _{u \in \mathcal{C}\left(y_{0}^{\varepsilon}, T, \varepsilon, M\right)}\|\rho u\|_{L^{2}(0, T)}\right\}
$$

larger than $K(\varepsilon, T, M)$ leading a priori to an upper bound $T_{M, \rho}$ of $T_{M}$. Since $\rho^{-1}$ vanishes only at time $T$, we suspect that the minimal time of uniform controllability $T_{M, \rho}$ coincides with $T_{M}$.

b) Even if the introduction of weights like $\rho$ improves the numerical stability of the mixed formulation (26), it seems quite impossible to consider values of $T$ far from $T^{\star}$ : for instance, for $T=2 \sqrt{2} T^{\star}$ exhibited in [30] (see Theorem 2.6), the norm $\left\|y^{\varepsilon}(\cdot, T)\right\|_{H^{-1}(0, L)}$ in the uncontrolled situation, is for $\varepsilon=10^{-2}$, about $3.33 \times 10^{-17}$. Consequently, when the double precision is used, we achieve "numerically" zero. Resolution of (26) would then lead to $v^{\varepsilon}:=0$ on $(0, T)$ ! A possible way to avoid such pathologies is to preliminary consider a change of variables. We may write the solution $y^{\varepsilon}$ as follows, for any $\alpha, \gamma \in \mathbb{R}$,

$$
y^{\varepsilon}(x, t)=e^{\frac{M \alpha x}{2 \varepsilon}} e^{-\frac{\gamma M^{2} t}{4 \varepsilon}} z^{\varepsilon}(x, t), \quad \forall(x, t) \in Q_{T},
$$


leading to

$$
L_{\varepsilon} y^{\varepsilon}:=e^{\frac{M \alpha x}{2 \varepsilon}} e^{-\frac{\gamma M^{2} t}{4 \varepsilon}}\left(z_{t}^{\varepsilon}-\varepsilon z_{x x}^{\varepsilon}+M(1-\alpha) z_{x}^{\varepsilon}-\frac{M^{2}}{4 \varepsilon}\left(\gamma+\alpha^{2}-2 \alpha\right) z^{\varepsilon}\right) .
$$

Remark that $y^{\varepsilon}(\cdot, T)=0$ if and only if $z^{\varepsilon}(\cdot, T)=0$. Taking $1-\alpha$ small and $\frac{M^{2}}{4 \varepsilon}\left(\gamma+\alpha^{2}-2 \alpha\right) \geq 0$ allows to reduce the dissipation of the solution at time $T$ as $\varepsilon \rightarrow 0$ and therefore avoid the zero numeric effect. The extreme case is for $\alpha=\gamma=1$ leading to the equation $z_{t}^{\varepsilon}-\varepsilon z_{x x}^{\varepsilon}=0$. Within this change of variable, the cost of control is

$$
K^{2}(\varepsilon, T, M)=\sup _{z_{0}^{\varepsilon} \in L^{2}(0, L)} \frac{\left(\mathcal{A}_{\varepsilon} z_{0}^{\varepsilon}, z_{0}^{\varepsilon}\right)}{\left(e^{\frac{M \alpha x}{\varepsilon}} z_{0}^{\varepsilon}, z_{0}^{\varepsilon}\right)}
$$

where $\mathcal{A}_{\varepsilon}$ is the control operator defined by $\mathcal{A}_{\varepsilon}: z_{0}^{\varepsilon} \rightarrow-w^{\varepsilon}(\cdot, 0) \in L^{2}(0,1)$; here $w^{\varepsilon}$ solves the adjoint problem

$$
\left\{\begin{array}{lr}
-w_{t}^{\varepsilon}-\varepsilon w_{x x}^{\varepsilon}-M(1-\alpha) w_{x}^{\varepsilon}-\frac{M^{2}}{4 \varepsilon}\left(\gamma+\alpha^{2}-2 \alpha\right) w^{\varepsilon}=0, & (x, t) \in Q_{T}, \\
w^{\varepsilon}(0, t)=w(L, t)=0, & t \in(0, T), \\
w^{\varepsilon}(x, T)=w_{T}^{\varepsilon}(x), & x \in(0, L),
\end{array}\right.
$$

with $w_{T}^{\varepsilon} \in H_{0}^{1}(0, L)$, the minimizer of the functional

$$
J^{\star}\left(w_{T}^{\varepsilon}\right):=\frac{1}{2} \int_{0}^{T} \varepsilon^{2} e^{\frac{\gamma M^{2} t}{2 \varepsilon}}\left(w_{x}^{\varepsilon}\right)^{2}(0, t) d t+\left(z_{0}^{\varepsilon}, w^{\varepsilon}(\cdot, 0)\right)_{L^{2}(0, L)} .
$$

The corresponding control of minimal $L^{2}\left(0, T ; e^{-\frac{\gamma M^{2} t}{4 \varepsilon}}\right)$-norm for the variable $z^{\varepsilon}$ is given by $v_{\varepsilon, z^{\varepsilon}}:=\varepsilon e^{\frac{\gamma M^{2} t}{2 \varepsilon}} w_{x}^{\varepsilon}(1, \cdot)$. The optimality conditions for $J^{\star}$ lead to a mixed formulation similar to 21). The introduction of appropriate parameters $\alpha$ and $\gamma$ allows to avoid the effect of the transport term; on the other hand, the change of variables make appear explicitly in the formulation some exponential functions which may lead to numerical overflow for small values of $\varepsilon$.

c) Another numerical strategy, employed in [37, is to use a spectral expansion of the adjoint solution $\varphi^{\varepsilon}$ of (8) (with $L=1$ here):

$$
\varphi^{\varepsilon}(x, t)=e^{-\frac{M x}{2 \varepsilon}} \sum_{k>0} \alpha_{k} e^{-\lambda_{\varepsilon, k}(T-t)} \sin (k \pi x), \quad \lambda_{\varepsilon, k}:=\varepsilon k^{2} \pi^{2}+\frac{M^{2}}{4 \varepsilon}
$$

with $\left\{\alpha_{k}\right\}_{k>0} \in \mathbb{L}(\varepsilon, M, T)$ such that $\varphi(x, 0)$ be in $L^{2}(0,1)$, equivalently

$$
\mathbb{L}(\varepsilon, M, T):=\left\{\left\{\alpha_{p}\right\}_{p>0} \in \mathbb{R}, \sum_{p, q>0} \alpha_{p} \alpha_{q} e^{-\left(\lambda_{\varepsilon, k}+\lambda_{\varepsilon, p}\right) T} \frac{32 \varepsilon^{3} M(p \pi)(q \pi)\left(1-e^{-\frac{M}{\varepsilon}}(-1)^{p+q}\right)}{\left(a_{p, q}^{2}-b_{p, q}^{2}\right)}<\infty\right\}
$$

with $a_{p, q}:=4\left(M^{2}+\varepsilon^{2}\left((p \pi)^{2}+(q \pi)^{2}\right)\right)$ and $b_{p, q}:=8 \varepsilon^{2}(p \pi)(q \pi)$. The characterization 14 of the control with $v_{\varepsilon}=\varepsilon \varphi_{x}^{\varepsilon}(0, \cdot)$ then rewrites as follows: find $\left\{\alpha_{k}\right\}_{k \geq 1} \in \mathbb{L}(\varepsilon, M, T)$ such that

$$
\varepsilon^{2} \sum_{k, p>0} \alpha_{k} \bar{\alpha}_{p}(k \pi)(p \pi) \frac{1-e^{-\left(\lambda_{\varepsilon, p}+\lambda_{\varepsilon, k}\right) T}}{\lambda_{\varepsilon, p}+\lambda_{\varepsilon, k}}+\sum_{k>0} \bar{\alpha}_{k} e^{-\lambda_{\varepsilon, k} T} \sum_{p>0} \beta_{p} M_{p, k}=0, \quad \forall\left\{\overline{\alpha_{k}}\right\}_{k>0} \in \mathbb{L}(\varepsilon, M, T),
$$

with $y_{0}^{\varepsilon}(x):=\sum_{p>0} \beta_{p}^{\varepsilon} \sin (p \pi x)$ and $M_{p, q}:=\int_{0}^{1} e^{-\frac{M x}{2 \varepsilon}} \sin (p \pi x) \sin (q \pi x) d x$. The use of symbolic computations with large digit numbers may allow to solve (32) with robustness, as done in [37] (we also refer to [4) in the context of the heat equation $(\varepsilon=1$ and $M=0)$. This remains however duable for not so small values of $\varepsilon$.

In the next section, we examine a more theoretical approach based on an asymptotic analysis with respect to the parameter $\varepsilon$. 


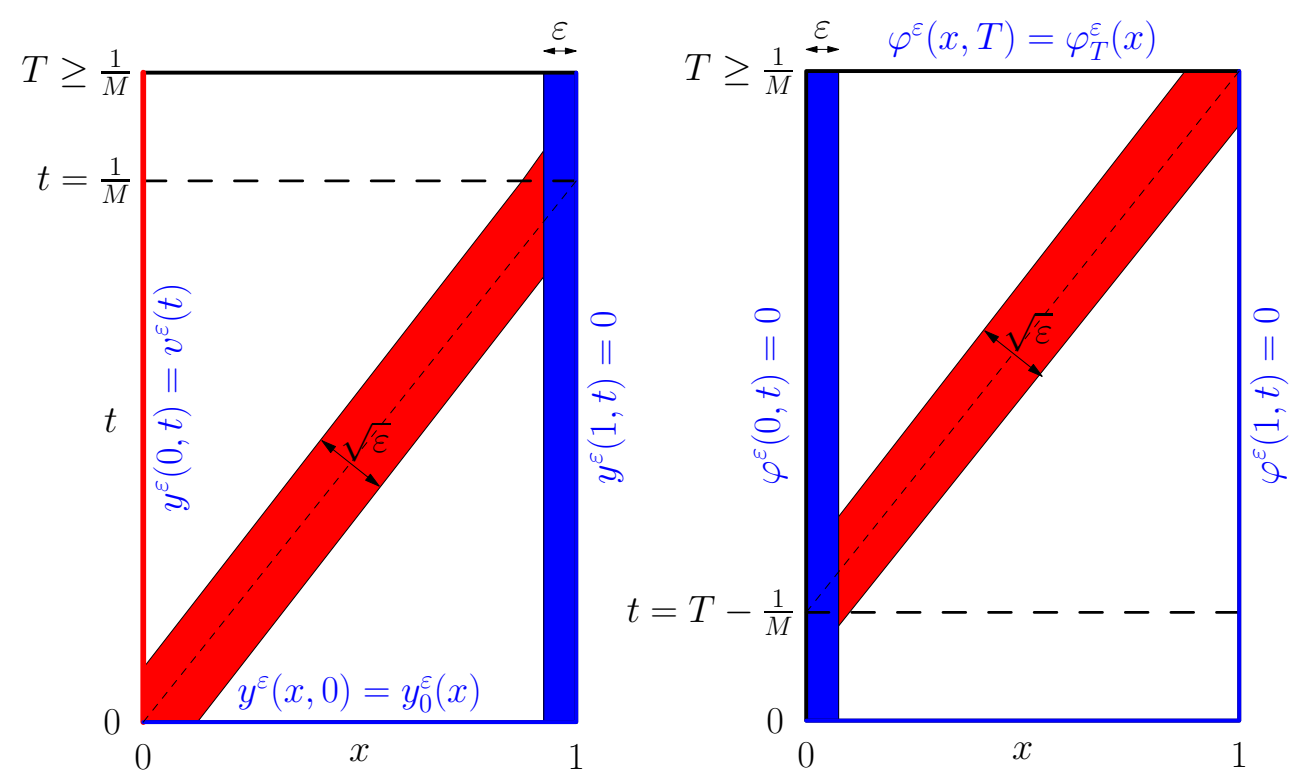

Figure 9: Boundary layer zones for $y^{\varepsilon}$ (left) and $\varphi^{\varepsilon}$ (right) in the case $M>0$.

\section{Asymptotic analysis with respect to $\varepsilon$}

In the spirit of the book [28, one possibility to better understand the behavior of the $\operatorname{cost} K(\varepsilon, T, M)$ is to perform an asymptotic analysis of the optimality system associated to the control of minimal $L^{2}$-norm. It couples the direct and adjoint solutions $y^{\varepsilon}$ and $\varphi^{\varepsilon}$ as follows :

$$
\left\{\begin{array}{lr}
L_{\varepsilon} y^{\varepsilon}=0, \quad L_{\varepsilon}^{\star} \varphi^{\varepsilon}=0, & (x, t) \in Q_{T}, \\
y^{\varepsilon}(x, 0)=y_{0}^{\varepsilon}(x), & x \in(0, L), \\
v^{\varepsilon}(t)=y^{\varepsilon}(0, t)=\varepsilon \varphi_{x}^{\varepsilon}(0, t), & t \in(0, T), \\
y^{\varepsilon}(L, t)=0, . & t \in(0, T), \\
\varphi^{\varepsilon}(0, t)=\varphi^{\varepsilon}(L, t)=0, & t \in(0, T), \\
-\beta(\varepsilon) \varphi_{x x}^{\varepsilon}(\cdot, T)+y^{\varepsilon}(\cdot, T)=0, & x \in(0, L) .
\end{array}\right.
$$

The asymptotic analysis with respect to $\varepsilon$ of such system is quite involved both for $M>0$ and $M<0$. Take for instance $M>0$. As $\varepsilon$ goes to zero, the direct solution $y^{\varepsilon}$ exhibits a boundary layer of size $\mathcal{O}(\varepsilon)$ at $x=L$ and a boundary layer of size $\mathcal{O}(\sqrt{\varepsilon})$ along the characteristic $\left\{(x, t) \in Q_{T}, L x-M t=0\right\}$. Therefore, two distincts boundary layers, with different sizes, appear and intersect (see Figure 9-Left)! Similarly, the adjoint solution $\varphi^{\varepsilon}$ exhibits a boundary layer of size $(\mathcal{O}(\varepsilon))$ at $x=0$ and a boundary layer of size $(\mathcal{O}(\sqrt{\varepsilon}))$ along the characteristic $\left\{(x, t) \in Q_{T}, L x-M(t-T)-1=0\right\}$ (Figure 9-Right). In particular, the control $v^{\varepsilon}$ lives in a boundary layer for the adjoint solution. Moreover, even if such boundary layers are not responsible of the unexpected behavior of the cost $K$ mentioned in the previous section, they must be considered in the asymptotic analysis.

Let us point out that the boundary layer at $x=0$ and $x=L$ (in blue on Figure 9 -Left) are unavoidable (unless $\left.y_{0}^{\varepsilon}=0\right)$. On the other hand, the ones along the characteristics can be avoided assuming some compatibility conditions at the point $(x, t)=(0,0)$ between the initial condition $y_{0}^{\varepsilon}$ at $x=0$ and the control $v^{\varepsilon}$ at $t=0$. There is however no reason for the control of minimal $L^{2}$-norm to satisfy such conditions ! Similarly, at the point $(x, t)=(L, T)$, some properties must hold for the adjoint solution $\varphi^{\varepsilon}$ that are not a priori encoded in the optimality system 33 .

In the next subsection, we perform the asymptotic analysis of the direct problem in $y^{\varepsilon}$, assuming appropriate compatibility conditions at the point $(x, t)=(0,0)$. This leads to quite involved but interesting developments, independently of the controllability issue. We consider the case $M>0$. In order to simplify notations, we also 
take $L=1$ so that $T^{\star}=1 / M$. We emphasize again that in view of the behavior of the free solution stated in Lemma 2.1, such asymptotic analysis should be as fine as possible in order to distinguish null and approximate controllability property. For this reason, the controllability time of interest for which we may capture some phenomenon is $T=T^{\star}$.

\subsection{Matched asymptotic expansions and approximate solutions}

In this section we apply the method of matched asymptotic expansions to construct approximate solutions of problem (1). We refer to [24, 39, 40] for a general presentation of the method. Then we apply the same procedure to construct asymptotic approximate solutions of the adjoint solution $\varphi^{\varepsilon}$, see problem (33).

Let us consider the problem

$$
\left\{\begin{array}{lc}
y_{t}^{\varepsilon}-\varepsilon y_{x x}^{\varepsilon}+M y_{x}^{\varepsilon}=0, & (x, t) \in Q_{T}, \\
y^{\varepsilon}(0, t)=v^{\varepsilon}(t), & t \in(0, T), \\
y^{\varepsilon}(1, t)=0, & t \in(0, T), \\
y^{\varepsilon}(x, 0)=y_{0}(x), & x \in(0,1),
\end{array}\right.
$$

where $y_{0}$ and $v^{\varepsilon}$ are given functions. We assume that $M>0$ and $v^{\varepsilon}$ is in the form $v^{\varepsilon}=\sum_{k=0}^{m} \varepsilon^{k} v^{k}$, the functions $v^{0}, v^{1}, \cdots, v^{m}$ being known. We construct an asymptotic approximation of the solution $y^{\varepsilon}$ of (34) by using the method of matched asymptotic expansions. We assume here that the initial condition $y^{\varepsilon}(x, 0)$ is independent of $\varepsilon$ but the procedure is very similar for $y^{\varepsilon}(\cdot, 0)$ of the form $y^{\varepsilon}(\cdot, 0)=\sum_{k=0}^{m} \varepsilon^{k} y_{0}^{k}$. The case $M<0$ can be treated similarly.

In the sequel, $c, c_{1}, c_{2}, \cdots$, will stand for generic constants that do not depend on $\varepsilon$. When the constants $c$, $c_{1}, c_{2}, \cdots$, depend in addition on some other parameter $p$ we will write $c_{p}, c_{1}(p), c_{2}(p), \cdots$

We also refer to [38] where a sensitivity analysis of an optimal control problem is performed an involving advection-diffusion equation.

\subsubsection{Formal asymptotic expansions}

Let us consider two formal asymptotic expansions of $y^{\varepsilon}$ :

- the outer expansion

$$
\sum_{k=0}^{m} \varepsilon^{k} y^{k}(x, t), \quad(x, t) \in Q_{T},
$$

- the inner expansion

$$
\sum_{k=0}^{m} \varepsilon^{k} Y^{k}(z, t), \quad z=\frac{1-x}{\varepsilon} \in\left(0, \varepsilon^{-1}\right), t \in(0, T) .
$$

We will construct outer and inner expansions which will be valid in the so-called outer and inner regions, respectively. Here the boundary layer (inner region) occurs near $x=1$, it is of $\mathcal{O}(\varepsilon)$ size, and the outer region is the subset of $(0,1)$ consisting of the points far from the boundary layer, it is of $\mathcal{O}(1)$ size. There is an intermediate region between them, with size $\mathcal{O}\left(\varepsilon^{\gamma}\right), \gamma \in(0,1)$. To construct an approximate solution we require that inner and outer expansions coincide in the intermediate region, then some conditions must be satisfied in that region by the inner and outer expansions. These conditions are the so-called matching asymptotic conditions.

$$
\begin{aligned}
& \text { Putting } \left.\sum_{k=0}^{m} \varepsilon^{k} y^{k}(x, t) \text { into equation } 34\right]_{1} \text {, the identification of the powers of } \varepsilon \text { yields } \\
& \qquad \begin{aligned}
\varepsilon^{0}: \quad y_{t}^{0}+M y_{x}^{0}=0, \\
\varepsilon^{k}: \quad y_{t}^{k}+M y_{x}^{k}=y_{x x}^{k-1}, \quad \text { for any } 1 \leq k \leq m .
\end{aligned}
\end{aligned}
$$


Taking the initial and boundary conditions into account we define $y^{0}$ and $y^{k}(1 \leq k \leq m)$ as functions satisfying the transport equations, respectively,

$$
\left\{\begin{array}{l}
y_{t}^{0}+M y_{x}^{0}=0, \quad(x, t) \in Q_{T} \\
y^{0}(0, t)=v^{0}(t), \\
y^{0}(x, 0)=y_{0}(x), \quad x \in(0, T)
\end{array}\right.
$$

and

$$
\begin{cases}y_{t}^{k}+M y_{x}^{k}=y_{x x}^{k-1}, & (x, t) \in Q_{T}, \\ y^{k}(0, t)=v^{k}(t), & t \in(0, T), \\ y^{k}(x, 0)=0, & x \in(0,1) .\end{cases}
$$

The solution of $(35)$ is given by

$$
y^{0}(x, t)= \begin{cases}y_{0}(x-M t), & x>M t \\ v^{0}\left(t-\frac{x}{M}\right), & x<M t .\end{cases}
$$

Using the method of characteristics we find that, for any $1 \leq k \leq m$,

$$
y^{k}(x, t)= \begin{cases}\int_{0}^{t} y_{x x}^{k-1}(x+(s-t) M, s) d s, & x>M t \\ v^{k}\left(t-\frac{x}{M}\right)+\int_{0}^{x / M} y_{x x}^{k-1}\left(s M, t-\frac{x}{M}+s\right) d s, & x<M t .\end{cases}
$$

Remark 9 Actually, above the first characteristic, we may express explicitly each function $y^{k}$ in term of the $v^{j}, j=0, \cdots, k$, which is very valuable in our controllability context. We verify that we have explicitly

$$
y^{1}(x, t)= \begin{cases}t y_{0}^{(2)}(x-M t), & x>M t, \\ v^{1}\left(t-\frac{x}{M}\right)+\frac{x}{M^{3}}\left(v^{0}\right)^{(2)}\left(t-\frac{x}{M}\right), & x<M t\end{cases}
$$

and

$$
y^{2}(x, t)= \begin{cases}\frac{t^{2}}{2} y_{0}^{(4)}(x-M t), & x>M t, \\ v^{2}\left(t-\frac{x}{M}\right)+\frac{x}{M^{3}}\left(v^{1}\right)^{(2)}\left(t-\frac{x}{M}\right) & \\ -\frac{2 x}{M^{5}}\left(v^{0}\right)^{(3)}\left(t-\frac{x}{M}\right)+\frac{x^{2}}{2 M^{6}}\left(v^{0}\right)^{(4)}\left(t-\frac{x}{M}\right), & x<M t .\end{cases}
$$

Here and in the sequel, $f^{(i)}$ denotes the derivative of order $i$ of the real function $f$.

Now we turn back to the construction of the inner expansion. Putting $\sum_{k=0}^{m} \varepsilon^{k} Y^{k}(z, t)$ into equation 34$)_{1}$, the identification of the powers of $\varepsilon$ yields

$$
\begin{array}{ll}
\varepsilon^{-1}: \quad Y_{z z}^{0}(z, t)+M Y_{z}^{0}(z, t)=0, \\
\varepsilon^{k-1}: \quad Y_{z z}^{k}(z, t)+M Y_{z}^{k}(z, t)=Y_{t}^{k-1}(z, t), \quad \text { for any } 1 \leq k \leq m .
\end{array}
$$

We impose that $Y^{k}(0, t)=0$ for any $0 \leq k \leq m$. To get the asymptotic matching conditions we write that, for any fixed $t$ and large $z$,

$$
\begin{aligned}
& Y^{0}(z, t)+\varepsilon Y^{1}(z, t)+\varepsilon^{2} Y^{2}(z, t)+\cdots+\varepsilon^{m} Y^{m}(z, t) \\
& =y^{0}(x, t)+\varepsilon y^{1}(x, t)+\varepsilon^{2} y^{2}(x, t)+\cdots+\varepsilon^{m} y^{m}(x, t)+\mathcal{O}\left(\varepsilon^{m+1}\right) .
\end{aligned}
$$


Rewriting the right-hand side of the above equality in terms of $z, t$ and using Taylor expansions we have

$$
\begin{aligned}
& Y^{0}(z, t)+\varepsilon Y^{1}(z, t)+\varepsilon^{2} Y^{2}(z, t)+\cdots+\varepsilon^{m} Y^{m}(z, t) \\
&= y^{0}(1-\varepsilon z, t)+\varepsilon y^{1}(1-\varepsilon z, t)+\varepsilon^{2} y^{2}(1-\varepsilon z, t)+\cdots+\varepsilon^{m} y^{m}(1-\varepsilon z, t)+\mathcal{O}\left(\varepsilon^{m+1}\right) \\
&= y^{0}(1, t)+y_{x}^{0}(1, t)(-\varepsilon z)+\frac{1}{2} y_{x x}^{0}(1, t)(\varepsilon z)^{2}+\cdots+\frac{1}{m !}\left(y^{0}\right)_{x}^{(m)}(1, t)(-\varepsilon z)^{m} \\
&+\varepsilon\left(y^{1}(1, t)+y_{x}^{1}(1, t)(-\varepsilon z)+\frac{1}{2} y_{x x}^{1}(1, t)(\varepsilon z)^{2}+\cdots+\frac{1}{(m-1) !}\left(y^{0}\right)_{x}^{(m-1)}(1, t)(-\varepsilon z)^{m-1}\right) \\
&+\cdots \cdots+\varepsilon^{m} y^{m}(1, t)+\mathcal{O}\left(\varepsilon^{m+1}\right) .
\end{aligned}
$$

Therefore the matching conditions read

$$
\begin{aligned}
& Y^{0}(z, t) \sim Q^{0}(z, t):=y^{0}(1, t), \quad \text { as } z \rightarrow+\infty \\
& Y^{1}(z, t) \sim Q^{1}(z, t):=y^{1}(1, t)-y_{x}^{0}(1, t) z, \quad \text { as } z \rightarrow+\infty, \\
& Y^{2}(z, t) \sim Q^{2}(z, t):=y^{2}(1, t)-y_{x}^{1}(1, t) z+\frac{1}{2} y_{x x}^{0}(1, t) z^{2}, \quad \text { as } z \rightarrow+\infty \\
& \cdots \\
& Y^{m}(z, t) \sim Q^{m}(z, t):=y^{m}(1, t)-y_{x}^{m-1}(1, t) z+\frac{1}{2} y_{x x}^{m-2}(1, t) z^{2}+\cdots+\frac{1}{m !}\left(y^{0}\right)_{x}^{(m)}(1, t)(-z)^{m}, \\
& \text { as } z \rightarrow+\infty .
\end{aligned}
$$

We thus define $Y^{0}$ as a solution of

$$
\left\{\begin{array}{lr}
Y_{z z}^{0}(z, t)+M Y_{z}^{0}(z, t)=0, & (z, t) \in(0,+\infty) \times(0, T), \\
Y^{0}(0, t)=0, & t \in(0, T), \\
\lim _{z \rightarrow+\infty} Y^{0}(z, t)=\lim _{x \rightarrow 1} y^{0}(x, t), & t \in(0, T) .
\end{array}\right.
$$

The last condition in $(42)$ is the matching asymptotic condition. The general solution of $\sqrt{42})_{1},(42)_{2}$ is $Y^{0}(z, t)=$ $C(t)\left(1-e^{-M z}\right)$, where $C(t)$ is an arbitrary constant. The matching condition allows to find $C(t)=y^{0}(1, t)$, leading to

$$
Y^{0}(z, t)=y^{0}(1, t)\left(1-e^{-M z}\right), \quad(z, t) \in(0,+\infty) \times(0, T) .
$$

Next we determine the general solution of

$$
\left\{\begin{array}{lr}
Y_{z z}^{1}(z, t)+M Y_{z}^{1}(z, t)=y_{t}^{0}(1, t)\left(1-e^{-M z}\right), & (z, t) \in(0,+\infty) \times(0, T), \\
Y^{1}(0, t)=0, & t \in(0, T) .
\end{array}\right.
$$

We find

$$
Y^{1}(z, t)=\left(C(t)-y_{x}^{0}(1, t) z\right)+e^{-M z}\left(-C(t)-y_{x}^{0}(1, t) z\right),
$$

where $C(t)$ is an arbitrary constant. We determine $C(t)$ by using the matching asymptotic condition

$$
\lim _{z \rightarrow+\infty}\left[Y^{1}(z, t)-Q^{1}(z, t]=0, \quad t \in(0, T)\right.
$$

which gives

$$
Y^{1}(z, t)=\left(y^{1}(1, t)-y_{x}^{0}(1, t) z\right)+e^{-M z}\left(-y^{1}(1, t)-y_{x}^{0}(1, t) z\right) .
$$

The function $Y^{2}$ is defined as a solution of

$$
\left\{\begin{array}{lr}
Y_{z z}^{2}(z, t)+M Y_{z}^{2}(z, t)=Y_{t}^{1}(z, t), & (z, t) \in(0,+\infty) \times(0, T), \\
Y^{2}(0, t)=0, & t \in(0, T), \\
\lim _{z \rightarrow+\infty}\left[Y^{2}(z, t)-Q^{2}(z, t]=0,\right. & t \in(0, T) .
\end{array}\right.
$$


We obtain

$$
Y^{2}(z, t)=\left(y^{2}(1, t)-y_{x}^{1}(1, t) z+y_{x x}^{0}(1, t) \frac{z^{2}}{2}\right)+e^{-M z}\left(-y^{2}(1, t)-y_{x}^{1}(1, t) z-y_{x x}^{0}(1, t) \frac{z^{2}}{2}\right) .
$$

For $1 \leq k \leq m$, the function $Y^{k}$ is defined iteratively as the solution of

$$
\left\{\begin{array}{lr}
Y_{z z}^{k}(z, t)+M Y_{z}^{k}(z, t)=Y_{t}^{k-1}(z, t), & (z, t) \in(0,+\infty) \times(0, T), \\
Y^{k}(0, t)=0, & t \in(0, T), \\
\lim _{z \rightarrow+\infty}\left[Y^{k}(z, t)-Q^{k}(z, t)\right]=0, & t \in(0, T) .
\end{array}\right.
$$

\subsubsection{Second order approximation}

Here we take $m=2$. The outer expansion is $\sum_{k=0}^{2} \varepsilon^{k} y^{k}(x, t)$, where $y^{0}$ and $y^{k}(k=1,2)$ are given by (37) and (38), respectively, and the inner expansion is $\sum_{k=0}^{2} \varepsilon^{k} Y^{k}(z, t)$, where $Y^{0}, Y^{1}$ and $Y^{2}$ are given by (43), 444) and 45, respectively. We introduce a $C^{\infty}$ cut-off function $\mathcal{X}: \mathbb{R} \rightarrow[0,1]$ such that

$$
\mathcal{X}(s)= \begin{cases}1, & s \geq 2 \\ 0, & s \leq 1\end{cases}
$$

and define, for $\gamma \in(0,1)$, the function $\mathcal{X}_{\varepsilon}:[0,1] \rightarrow[0,1]$, plotted on Figure 10 , by

$$
\mathcal{X}_{\varepsilon}(x)=\mathcal{X}\left(\frac{1-x}{\varepsilon^{\gamma}}\right)
$$

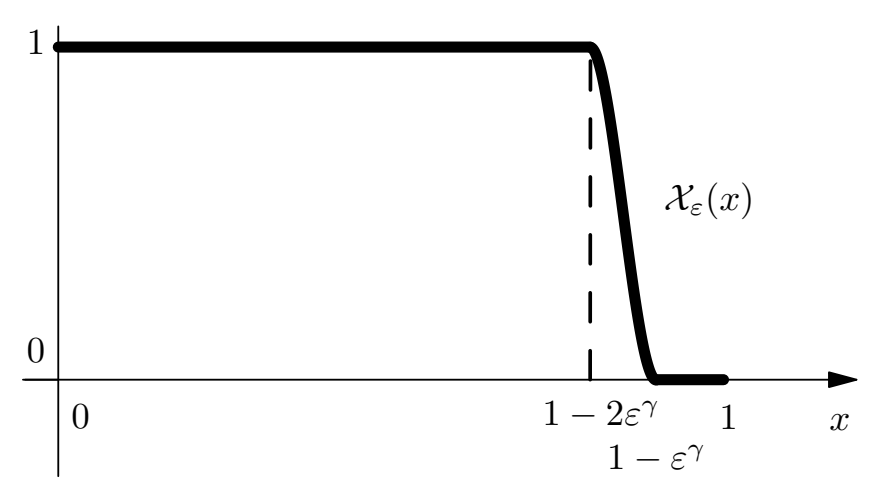

Figure 10: The function $\mathcal{X}_{\varepsilon}$.

Then we introduce the function $w_{2}^{\varepsilon}$ by

$$
w_{2}^{\varepsilon}(x, t)=\mathcal{X}_{\varepsilon}(x) \sum_{k=0}^{2} \varepsilon^{k} y^{k}(x, t)+\left(1-\mathcal{X}_{\varepsilon}(x)\right) \sum_{k=0}^{2} \varepsilon^{k} Y^{k}\left(\frac{1-x}{\varepsilon}, t\right), \quad(x, t) \in Q_{T},
$$

defined to be the second order asymptotic approximation of the solution $y^{\varepsilon}$ of (34). To justify all the computations we will perform we need some regularity assumptions on the data $y_{0}, v^{0}, v^{1}$ and $v^{2}$. We have the following result.

Lemma 4.1 (i) Assume that $y_{0} \in C^{5}[0,1], v^{0} \in C^{5}[0, T]$ and the following $C^{5}$-matching conditions are satisfied

$$
M^{p}\left(y_{0}\right)^{(p)}(0)+(-1)^{p+1}\left(v^{0}\right)^{(p)}(0)=0, \quad 0 \leq p \leq 5 .
$$


Then the function $y^{0}$ defined by $(37)$ belongs to $C^{5}\left(\overline{Q_{T}}\right)$.

(ii) Additionally, assume that $v^{1} \in C^{3}[0, T], v^{2} \in C^{1}[0, T]$ and the following $C^{3}$ and $C^{1}$-matching conditions are satisfied, respectively,

$$
\begin{aligned}
& \left\{\begin{array}{l}
v^{1}(0)=0, \quad\left(v^{1}\right)^{(1)}(0)=M^{-2}\left(v^{0}\right)^{(2)}(0)=y_{0}^{(2)}(0), \\
\left(v^{1}\right)^{(2)}(0)=2 M^{-2}\left(v^{0}\right)^{(3)}(0)=-2 M y_{0}^{(3)}(0), \\
\left(v^{1}\right)^{(3)}(0)=3 M^{-2}\left(v^{0}\right)^{(4)}(0)=3 M^{2} y_{0}^{(4)}(0),
\end{array}\right. \\
& v^{2}(0)=0, \quad\left(v^{2}\right)^{(1)}(0)=0 .
\end{aligned}
$$

Then the function $y^{1}$ defined by (38) (with $k=1$ ) belongs to $C^{3}\left(\overline{Q_{T}}\right)$, and the function $y^{2}$ defined by (38) (with $k=2$ ) belongs to $C^{1}\left(\overline{Q_{T}}\right)$.

Proof. (i) According to the explicit form (37), it suffices to match the partial derivative of $y^{0}$ on the characteristic line $\{(x, t), x-M t=0\}$. Differentiating (37) $p$ times $(p \leq 5)$ with respect to $x$ we have

$$
\frac{\partial^{p} y^{0}}{\partial x^{p}}(x, t)= \begin{cases}y_{0}^{(p)}(x-M t), & x>M t \\ \frac{(-1)^{p}}{M^{p}}\left(v^{0}\right)^{(p)}\left(t-\frac{x}{M}\right), & x<M t .\end{cases}
$$

Matching the expressions of $\frac{\partial^{p} y^{0}}{\partial x^{p}}$ upper and under the characteristic line $\{(x, t), x-M t=0\}$ gives $(50)$ and ensures the continuity of $\frac{\partial^{p} y^{0}}{\partial x^{p}}$ in $\overline{Q_{T}}$. Differentiating (37) $p$ times with respect to $t$ we have

$$
\frac{\partial^{p} y^{0}}{\partial t^{p}}(x, t)= \begin{cases}(-1)^{p} M^{p} y_{0}^{(p)}(x-M t), & x>M t \\ \left(v^{0}\right)^{(p)}\left(t-\frac{x}{M}\right), & x<M t\end{cases}
$$

then we see that the continuity of $\frac{\partial^{p} y^{0}}{\partial t^{p}}$ holds under condition 50 . Using equation 35 we easily verify that the mixed partial derivatives, of order $p \leq 5$, of $y^{0}$ are continuous under condition (50).

(ii) Arguing as previously, using formula (39) and equation (36) (with $k=1$ ) we find the matching conditions (51). Then, using formula (40) and equation (36) (with $k=2$ ) we find the matching conditions (52).

Lemma 4.2 Let $w_{2}^{\varepsilon}$ be the function defined by 49. Assume that the assumptions of Lemma 4.1 hold true. Then there is a constant $c$ independent of $\varepsilon$ such that

$$
\left\|L_{\varepsilon}\left(w_{2}^{\varepsilon}\right)\right\|_{C\left([0, T] ; L^{2}(0,1)\right)} \leq c \varepsilon^{\frac{5 \gamma}{2}} .
$$

Proof. A straightforward calculation gives $L_{\varepsilon}\left(w_{2}^{\varepsilon}\right)(x, t)=\sum_{i=1}^{5} I_{\varepsilon}^{i}(x, t)$, with

$$
\begin{aligned}
& I_{\varepsilon}^{1}(x, t)=-\varepsilon^{3} y_{x x}^{2}(x, t) \mathcal{X}_{\varepsilon}(x) \\
& I_{\varepsilon}^{2}(x, t)=\varepsilon^{2}\left(1-\mathcal{X}_{\varepsilon}(x)\right) Y_{t}^{2}\left(\frac{1-x}{\varepsilon}, t\right) \\
& I_{\varepsilon}^{3}(x, t)=M \mathcal{X}^{\prime}\left(\frac{1-x}{\varepsilon^{\gamma}}\right) \varepsilon^{-\gamma}\left(\sum_{k=0}^{2} \varepsilon^{k} Y^{k}\left(\frac{1-x}{\varepsilon}, t\right)-\sum_{k=0}^{2} \varepsilon^{k} y^{k}(x, t)\right), \\
& I_{\varepsilon}^{4}(x, t)=\mathcal{X}^{\prime \prime}\left(\frac{1-x}{\varepsilon^{\gamma}}\right) \varepsilon^{1-2 \gamma}\left(\sum_{k=0}^{2} \varepsilon^{k} Y^{k}\left(\frac{1-x}{\varepsilon}, t\right)-\sum_{k=0}^{2} \varepsilon^{k} y^{k}(x, t)\right), \\
& I_{\varepsilon}^{5}(x, t)=2 \mathcal{X}^{\prime}\left(\frac{1-x}{\varepsilon^{\gamma}}\right) \varepsilon^{1-\gamma}\left(\varepsilon^{-1} \sum_{k=0}^{2} \varepsilon^{k} Y_{z}^{k}\left(\frac{1-x}{\varepsilon}, t\right)+\sum_{k=0}^{2} \varepsilon^{k} y_{x}^{k}(x, t)\right) .
\end{aligned}
$$


Clearly,

$$
\left\|I_{\varepsilon}^{1}\right\|_{C\left([0, T] ; L^{2}(0,1)\right)} \leq \varepsilon^{3}\left\|y_{x x}^{2}\right\|_{C\left([0, T] ; L^{2}(0,1)\right)} \leq c \varepsilon^{3},
$$

and

$$
\begin{aligned}
\left\|I_{\varepsilon}^{2}\right\|_{C\left([0, T] ; L^{2}(0,1)\right)} & \leq \varepsilon^{2}\left\|\left(1-\mathcal{X}_{\varepsilon}(x)\right) Y_{t}^{2}\left(\frac{1-x}{\varepsilon}, t\right)\right\|_{C\left([0, T] ; L^{2}(0,1)\right)} \\
& \leq \varepsilon^{2} \max _{t \in[0, T]}\left(\int_{1-2 \varepsilon^{\gamma}}^{1}\left|Y_{t}^{2}\left(\frac{1-x}{\varepsilon}, t\right)\right|^{2} d x\right)^{1 / 2}
\end{aligned}
$$

Using a change of variable we have

$$
\left(\int_{1-2 \varepsilon^{\gamma}}^{1}\left|Y_{t}^{2}\left(\frac{1-x}{\varepsilon}, t\right)\right|^{2} d x\right)^{1 / 2}=\left(\varepsilon \int_{0}^{\frac{2 \varepsilon \gamma}{\varepsilon}}\left|Y_{t}^{2}(z, t)\right|^{2} d z\right)^{1 / 2} .
$$

Thanks to the explicit form 45 we have, for $0<\varepsilon \leq \varepsilon_{0}$ small enough,

$$
\max _{t \in[0, T]}\left(\varepsilon \int_{0}^{\frac{2 \varepsilon^{\gamma}}{\varepsilon}}\left|Y_{t}^{2}(z, t)\right|^{2} d z\right)^{1 / 2} \leq c\left\|y_{x x t}^{0}\right\|_{C([0,1] \times[0, T])}\left(\varepsilon \int_{0}^{\frac{2 \varepsilon \gamma}{\varepsilon}} z^{4} d z\right)^{1 / 2} \leq c \varepsilon^{-2} \varepsilon^{\frac{5 \gamma}{2}} .
$$

It results that

$$
\left\|I_{\varepsilon}^{2}\right\|_{C\left([0, T] ; L^{2}(0,1)\right)} \leq c \varepsilon^{\frac{5 \gamma}{2}} .
$$

Using Taylor expansions, for $1-x=\varepsilon z \rightarrow 0$, we have

$$
\sum_{k=0}^{2} \varepsilon^{k} y^{k}(x, t)=\sum_{k=0}^{2} \varepsilon^{k} y^{k}(1-\varepsilon z, t)=\sum_{k=0}^{2} \varepsilon^{k}\left(\sum_{i=0}^{2-k} \frac{1}{i !} \frac{\partial^{i} y^{k}}{\partial x^{i}}(1, t)(-\varepsilon z)^{i}\right)+\varepsilon^{2} \mathcal{O}((\varepsilon z)) .
$$

Since

$$
Y^{k}(z, t)=Q^{k}(z, t)+e^{-M z} P^{k}(z, t), \quad(z, t) \in(0,+\infty) \times(0, t),
$$

with

$$
P^{k}(z, t)=-\sum_{i=0}^{k} \frac{\partial^{i} y^{k}}{\partial x^{i}}(1, t) z^{i}, \quad Q^{k}(z, t)=\sum_{i=0}^{k}(-1)^{i} \frac{\partial^{i} y^{k}}{\partial x^{i}}(1, t) z^{i}
$$

we have

$$
\sum_{k=0}^{2} \varepsilon^{k} Y^{k}(z, t)-\sum_{k=0}^{2} \varepsilon^{k} y^{k}(1-\varepsilon z, t)=\varepsilon^{2} \mathcal{O}((\varepsilon z))+e^{-M z} \sum_{k=0}^{2} \varepsilon^{k} P^{k}(z, t) .
$$

Using the previous estimate we have

$$
\begin{aligned}
& \left\|I_{\varepsilon}^{3}\right\|_{C\left([0, T] ; L^{2}(0,1)\right)} \\
& \quad=M \varepsilon^{-\gamma}\left\|\mathcal{X}^{\prime}\left(\frac{1-x}{\varepsilon^{\gamma}}\right)\left(\sum_{k=0}^{2} \varepsilon^{k} Y^{k}\left(\frac{1-x}{\varepsilon}, t\right)-\sum_{k=0}^{2} \varepsilon^{k} y^{k}(1-\varepsilon z, t)\right)\right\|_{C\left([0, T] ; L^{2}(0,1)\right)} \\
& \quad \leq c \varepsilon^{2-\gamma}\left(\int_{1-2 \varepsilon^{\gamma}}^{1-\varepsilon^{\gamma}}(1-x)^{2} d x\right)^{1 / 2} \leq c \varepsilon^{2+\frac{\gamma}{2}} .
\end{aligned}
$$

Similarly we have $\left\|I_{\varepsilon}^{4}\right\|_{C\left([0, T] ; L^{2}(0,1)\right)} \leq c \varepsilon^{3-\frac{\gamma}{2}}$. It results from t54 that

$$
\begin{aligned}
\varepsilon^{-1} \sum_{k=0}^{2} \varepsilon^{k} Y_{z}^{k}(z, t)+\sum_{k=0}^{2} \varepsilon^{k} y_{x}^{k}(1-\varepsilon z, t)= & \varepsilon \mathcal{O}((\varepsilon z))+\varepsilon^{-1} e^{-M z} \sum_{k=0}^{2} \varepsilon^{k} P_{z}^{k}(z, t) \\
& -\varepsilon^{-1} M e^{-M z} \sum_{k=0}^{2} \varepsilon^{k} P^{k}(z, t)
\end{aligned}
$$


Arguing as for $I_{\varepsilon}^{3}$ we find that

$$
\left\|I_{\varepsilon}^{5}\right\|_{C\left([0, T] ; L^{2}(0,1)\right)} \leq c \varepsilon^{2+\frac{\gamma}{2}} .
$$

Collecting the previous estimates, we obtain (53).

Let us now consider the initial layer corrector $\theta^{\varepsilon}$ defined as the solution of

$$
\left\{\begin{array}{lc}
\theta_{t}^{\varepsilon}-\varepsilon \theta_{x x}^{\varepsilon}+M \theta_{x}^{\varepsilon}=0, & (x, t) \in Q_{T} \\
\theta^{\varepsilon}(0, t)=\theta^{\varepsilon}(1, t)=0, & t \in(0, T) \\
\theta^{\varepsilon}(x, 0)=\theta_{0}^{\varepsilon}(x), & x \in(0,1)
\end{array}\right.
$$

with

$$
\theta_{0}^{\varepsilon}(x)=: y_{0}(x)-w_{2}^{\varepsilon}(x, 0)=\left(1-\mathcal{X}_{\varepsilon}(x)\right)\left(y_{0}(x)-\sum_{k=0}^{2} \varepsilon^{k} Y^{k}\left(\frac{1-x}{\varepsilon}, 0\right)\right), \quad x \in(0,1) .
$$

The following lemma gives an estimate of $\left\|\theta^{\varepsilon}(\cdot, t)\right\|_{L^{2}(0,1)}$.

Lemma 4.3 Let $\theta^{\varepsilon}$ be the solution of problem (55), (56). Assume $\gamma \in(0,1 / 2]$. Then there exists a constant $c$ independent of $\varepsilon$ such that

$$
\left\|\theta^{\varepsilon}(\cdot, t)\right\|_{L^{2}(0,1)} \leq c\left(\varepsilon^{\frac{1}{2}}+\varepsilon^{\frac{7 \gamma}{2}}\right) e^{-\frac{M^{2}}{2 \varepsilon^{\gamma}} t}, \quad \forall t \in[0, T] .
$$

Proof. By repeating the arguments in the proof of Lemma 2.1 with $\alpha=\varepsilon^{1-\gamma}$, we obtain the estimate

$$
\left\|\theta^{\varepsilon}(\cdot, t)\right\|_{L^{2}(0,1)} \leq c\left\|\theta_{0}^{\varepsilon}\right\|_{L^{2}\left(1-2 \varepsilon^{\gamma}, 1\right)} e^{-\frac{M^{2}}{2 \varepsilon^{\gamma} t}}, \quad \forall t \in[0, T] .
$$

Let us now give an estimate of $\left\|\theta_{0}^{\varepsilon}\right\|_{L^{2}\left(1-2 \varepsilon^{\gamma}, 1\right)}$. Using 443 - 45] it holds that $\theta_{0}^{\varepsilon}=a^{\varepsilon}+b^{\varepsilon}$, with

$$
\begin{aligned}
& a^{\varepsilon}(x)=\left(1-\mathcal{X}_{\varepsilon}(x)\right)\left(y_{0}(x)-y_{0}(1)+y_{0}^{(1)}(1)(\varepsilon z)-y_{0}^{(2)}(1) \frac{(\varepsilon z)^{2}}{2}\right), \\
& b^{\varepsilon}(x)=\left(1-\mathcal{X}_{\varepsilon}(x)\right) e^{-M z}\left(y_{0}(1)+y_{0}^{(1)}(1)(\varepsilon z)+y_{0}^{(2)}(1) \frac{(\varepsilon z)^{2}}{2}\right), \quad\left(z=\frac{1-x}{\varepsilon}\right) .
\end{aligned}
$$

Using Taylor's expansion, for $1-x=\varepsilon z \rightarrow 0$, we have

$$
a^{\varepsilon}(x)=\left(1-\mathcal{X}_{\varepsilon}(x)\right) \frac{(1-x)^{3}}{6} y_{0}^{(3)}(\zeta), \quad \zeta \in(x, 1),
$$

hence $\left\|a^{\varepsilon}\right\|_{L^{2}(0,1)} \leq c \varepsilon^{\frac{7 \gamma}{2}}$. Since

$$
\left\|b^{\varepsilon}\right\|_{L^{2}(0,1)}^{2} \leq \int_{1-2 \varepsilon^{\gamma}}^{1} e^{-2 M z}\left(y_{0}(1)+y_{0}^{(1)}(1)(\varepsilon z)+y_{0}^{(2)}(1) \frac{(\varepsilon z)^{2}}{2}\right)^{2} d x,
$$

we have $\left\|b^{\varepsilon}\right\|_{L^{2}(0,1)} \leq c \varepsilon^{\frac{1}{2}}$. It results that $\left\|\theta_{0}^{\varepsilon}\right\|_{L^{2}(0,1)} \leq c\left(\varepsilon^{\frac{7 \gamma}{2}}+\varepsilon^{\frac{1}{2}}\right)$ which with 558 imply the estimate 57 .

Let us now establish the following result.

Lemma 4.4 Let $y^{\varepsilon}$ be the solution of problem (34), let $w_{2}^{\varepsilon}$ be the function defined by 49 and let $\theta^{\varepsilon}$ be the solution of problem (55), (56). Assume that the assumptions of Lemma 4.1 hold true. Then there is a constant $c$, independent of $\varepsilon$, such that

$$
\left\|y^{\varepsilon}-w_{2}^{\varepsilon}-\theta^{\varepsilon}\right\|_{C\left([0, T] ; L^{2}(0,1)\right)} \leq c \varepsilon^{\frac{5 \gamma}{2}} .
$$


Proof. Let us consider the function $z^{\varepsilon}=y^{\varepsilon}-w_{2}^{\varepsilon}-\theta^{\varepsilon}$. By construction, it satisfies

$$
\left\{\begin{array}{lr}
L_{\varepsilon}\left(z^{\varepsilon}\right)=-L_{\varepsilon}\left(w_{2}^{\varepsilon}\right), & (x, t) \in Q_{T}, \\
z^{\varepsilon}(0, t)=z^{\varepsilon}(1, t)=0, & t \in(0, T), \\
z^{\varepsilon}(x, 0)=0, & x \in(0,1),
\end{array}\right.
$$

leading to the estimate $\left\|z^{\varepsilon}(\cdot, t)\right\|_{L^{2}(0,1)}^{2} \leq\left\|L_{\varepsilon}\left(w_{2}^{\varepsilon}\right)\right\|_{L^{2}\left(Q_{T}\right)}^{2} e^{t}$, for all $t \in(0, T]$. Lemma 4.2 then implies (??).

Using Lemmas 4.3 and 4.4 we immediately obtain the following result.

TheOREM 4.1 Let $y^{\varepsilon}$ be the solution of problem (34) and let $w_{2}^{\varepsilon}$ be the function defined by (49). Assume that the assumptions of Lemma 4.1 hold true and $\gamma \in(0,1 / 2]$. Then there exist two positive constants $c$ and $\varepsilon_{0}, c$ independent of $\varepsilon$, such that, for any $0<\varepsilon<\varepsilon_{0}$,

$$
\left\|y^{\varepsilon}(\cdot, t)-w_{2}^{\varepsilon}(\cdot, t)\right\|_{L^{2}(0,1)} \leq c \varepsilon^{\frac{5 \gamma}{2}}+c\left(\varepsilon^{\frac{1}{2}}+\varepsilon^{\frac{7 \gamma}{2}}\right) e^{-\frac{M^{2}}{2 \varepsilon^{\gamma}} t}, \quad \forall t \in[0, T] .
$$

Remark 10 We may also define the so-called composite approximations and avoid the use of the cut-off function $\mathcal{X}_{\varepsilon}$. For instance, at the first order, we define the approximation

$$
\widetilde{w}_{0}^{\varepsilon}(x, t)=y^{0}(x, t)-y^{0}(1, t) e^{-M z}, \quad(x, t) \in Q_{T}
$$

obtained by adding the inner and outer expansions $y^{0}$ and $Y^{0}$ and then subtracting their common part, here equal (in view of (41)) to $y^{0}(1, t)$. The difference $z_{0}^{\varepsilon}:=y^{\varepsilon}-\widetilde{w}_{0}^{\varepsilon}$ then solves

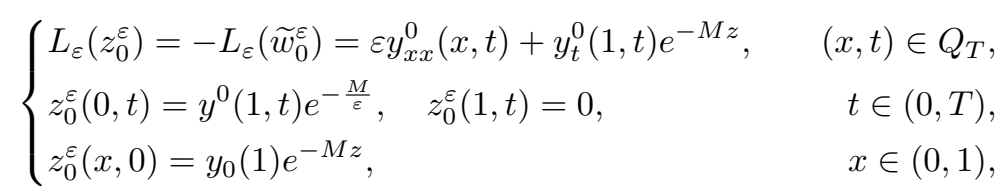

from which we deduce, using an estimate of the normal derivative (see (24)) to take into account the vanishing but nonzero Dirichlet condition at $x=0$, that $\left\|y^{\varepsilon}-\widetilde{w}_{0}^{\varepsilon}\right\|_{C\left([0, T] ; L^{2}(0,1)\right)}=\mathcal{O}\left(\varepsilon^{1 / 2}\right)$.

Similarly, at the next order, we define

$$
\widetilde{w}_{1}^{\varepsilon}(x, t)=\widetilde{w}_{0}^{\varepsilon}(x, t)+\varepsilon\left(y^{1}(x, t)-e^{-M z}\left(y^{1}(1, t)+y_{x}^{0}(1, t) z\right)\right), \quad(x, t) \in Q_{T},
$$

and compute that the difference $z_{1}^{\varepsilon}:=y^{\varepsilon}-\widetilde{w}_{1}^{\varepsilon}$ solves

$$
\left\{\begin{array}{lrl}
L_{\varepsilon}\left(z_{1}^{\varepsilon}\right)=\varepsilon^{2} y_{x x}^{1}(x, t)+\varepsilon e^{-M z}\left(y_{t}^{1}(1, t)+y_{x t}^{0}(1, t) z\right), & (x, t) \in Q_{T}, \\
z_{1}^{\varepsilon}(0, t)=\left(y^{0}(1, t)+y_{x}^{0}(1, t)+\varepsilon y^{1}(1, t)\right) e^{-\frac{M}{\varepsilon}}, & z_{0}^{\varepsilon}(1, t)=0, & t \in(0, T), \\
z_{1}^{\varepsilon}(x, 0)=\left(y_{0}(1)+y_{0}^{(1)}(\varepsilon z)\right) e^{-M z}, & x \in(0,1),
\end{array}\right.
$$

for which we deduce, arguing as in the proof of Lemma 4.3 that $\left\|y^{\varepsilon}-\widetilde{w}_{1}^{\varepsilon}\right\|_{C\left([0, T] ; L^{2}(0,1)\right)}=\mathcal{O}\left(\varepsilon^{3 / 2}\right)$. We do not go on further here with composite approximations and refer to [9] where it is employed in a similar context and in [2] to describe the interaction of this boundary layer at $x=1$ with the one occurring along the first characteristic $\left\{(x, t) \in Q_{T}, x-M t=0\right\}$.

\subsubsection{High order asymptotic approximation}

Here we construct an asymptotic approximation of the solution $y^{\varepsilon}$ of $(34)$ at any order $m$. The outer expansion is $\sum_{k=0}^{m} \varepsilon^{k} y^{k}(x, t)$, where the functions $y^{0}$ and $y^{k}(1 \leq k \leq m)$ are given by (37) and (38), respectively. The inner expansion is given by $\sum_{k=0}^{m} \varepsilon^{k} Y^{k}(z, t)$, where the function $Y^{0}$ is given by (43), and the function $Y^{k}(1 \leq k \leq m)$ is a solution of problem 46.

By induction over the parameter $k$, we obtain the following result (we refer to [1] for the proof) 
LEMMA 4.5 For any $1 \leq k \leq m$, the solution of problem 46 reads

$$
Y^{k}(z, t)=Q^{k}(z, t)+e^{-M z} P^{k}(z, t), \quad(z, t) \in(0,+\infty) \times(0, t)
$$

where

$$
P^{k}(z, t)=-\sum_{i=0}^{k} \frac{1}{i !} \frac{\partial^{i} y^{k-i}}{\partial x^{i}}(1, t) z^{i}, \quad Q^{k}(z, t)=\sum_{i=0}^{k} \frac{(-1)^{i}}{i !} \frac{\partial^{i} y^{k-i}}{\partial x^{i}}(1, t) z^{i}
$$

We then introduce the function

$$
w_{m}^{\varepsilon}(x, t)=\mathcal{X}_{\varepsilon}(x) \sum_{k=0}^{m} \varepsilon^{k} y^{k}(x, t)+\left(1-\mathcal{X}_{\varepsilon}(x)\right) \sum_{k=0}^{m} \varepsilon^{k} Y^{k}\left(\frac{1-x}{\varepsilon}, t\right),
$$

defined to be an asymptotic approximation at order $m$ of the solution $y^{\varepsilon}$ of (34). Function $\mathcal{X}_{\varepsilon}$ is defined on (48). Again, in order to justify the computations, we make the following regularity assumptions.

LEMMA 4.6 (i) Assume that $y_{0} \in C^{2 m+1}[0,1], v^{0} \in C^{2 m+1}[0, T]$ and the following $C^{2 m+1}$-matching conditions are satisfied

$$
M^{p}\left(y_{0}\right)^{(p)}(0)+(-1)^{p+1}\left(v^{0}\right)^{(p)}(0)=0, \quad 0 \leq p \leq 2 m+1 .
$$

Then the function $y^{0}$ defined by (37) belongs to $C^{2 m+1}\left(\overline{Q_{T}}\right)$.

(ii) Additionally, assume that $v^{k} \in C^{2(m-k)+1}[0, T]$, and the following $C^{2(m-k)+1}$-matching conditions are satisfied, respectively,

$$
\left(v^{k}\right)^{(p)}(0)=\sum_{i+j=p-1}(-1)^{i} M^{i} \frac{\partial^{p+1} y^{k-1}}{\partial x^{i+2} \partial t^{j}}(0,0), \quad 0 \leq p \leq 2(m-k)+1 .
$$

Then the function $y^{k}$ belongs to $C^{2(m-k)+1}\left(\overline{Q_{T}}\right)$.

Proof. (i) For the proof of (61) we refer to that of (50).

(ii) Using a change of variable we rewrite $(38)$ in the form

$$
y^{k}(x, t)= \begin{cases}\int_{0}^{t} y_{x x}^{k-1}(x+M(s-t), s) d s, & x>M t, \\ v^{k}\left(t-\frac{x}{M}\right)+\int_{t-x / M}^{t} y_{x x}^{k-1}(x+M(s-t), s) d s, & x<M t .\end{cases}
$$

For notational convenience we omit in the sequel the index $k$ and denote $y_{x x}^{k-1}=f$ so that (63) reads

$$
y(x, t)= \begin{cases}\int_{0}^{t} f(x+(s-t) M, s) d s, & x>M t \\ v\left(t-\frac{x}{M}\right)+\int_{t-x / M}^{t} f(x+M(s-t), s) d s, & x<M t\end{cases}
$$

Successive partial derivatives with respect to $x$ lead to the formulae:

$$
\frac{\partial^{p} y}{\partial x^{p}}(x, t)=\int_{0}^{t} \frac{\partial^{p} f}{\partial x^{p}}(x+M(s-t), s) d s \quad \text { for } x>M t,
$$

and

$$
\begin{aligned}
\frac{\partial^{p} y}{\partial x^{p}}(x, t)= & \frac{(-1)^{p}}{M^{p}} v^{(p)}\left(t-\frac{x}{M}\right)+\int_{t-x / M}^{t} \frac{\partial^{p} f}{\partial x^{p}}(x+M(s-t), s) d s \\
& +\sum_{i+j=p-1} \frac{(-1)^{j}}{M^{j+1}} \frac{\partial^{p-1} f}{\partial x^{i} \partial t^{j}}\left(0, t-\frac{x}{M}\right) \quad \text { for } x<M t .
\end{aligned}
$$


These formulae can be easily justified by induction. Then it results from 64 and 65 that $\frac{\partial^{p} y}{\partial x^{p}}$ is continuous in $\overline{Q_{T}}$ if

$$
\frac{(-1)^{p}}{M^{p}} v^{(p)}(0)=-\sum_{i+j=p-1} \frac{(-1)^{j}}{M^{j+1}} \frac{\partial^{p-1} f}{\partial x^{i} \partial t^{j}}(0,0),
$$

which is equivalent to 62 . Similar calculations allow to establish the formulae

$$
\begin{aligned}
\frac{\partial^{p} y}{\partial t^{p}}(x, t)= & (-1)^{p} M^{p} \int_{0}^{t} \frac{\partial^{p} f}{\partial x^{p}}(x+M(s-t), s) d s \\
& +\sum_{i+j=p-1}(-1)^{i} M^{i} \frac{\partial^{p-1} f}{\partial x^{i} \partial t^{j}}(x, t) \quad \text { for } x>M t
\end{aligned}
$$

and

$$
\begin{aligned}
\frac{\partial^{p} y}{\partial t^{p}}(x, t)= & v^{(p)}\left(t-\frac{x}{M}\right)+(-1)^{p} M^{p} \int_{t-x / M}^{t} \frac{\partial^{p} f}{\partial x^{p}}(x+M(s-t), s) d s \\
& +\sum_{i+j=p-1}(-1)^{i} M^{i}\left(\frac{\partial^{p-1} f}{\partial x^{i} \partial t^{j}}(x, t)-\frac{\partial^{p-1} f}{\partial x^{i} \partial t^{j}}\left(t-\frac{x}{M}\right)\right) \quad \text { for } x<M t .
\end{aligned}
$$

It results from 66 and 67 that $\frac{\partial^{p} y}{\partial t^{p}}$ is continuous in $\overline{Q_{T}}$ if

$$
v^{(p)}(0)=\sum_{i+j=p-1}(-1)^{i} M^{i} \frac{\partial^{p-1} f}{\partial x^{i} \partial t^{j}}(0,0),
$$

that is the condition 62. Using equation (36) we easily verify that the mixed partial derivatives, of order $0 \leq p \leq 2(m-k)+1$, of $y^{k}$ are continuous under condition 62 .

Remark 11 Let $m=2$. For $k=1$ the conditions $(62)$ read

$$
\begin{aligned}
& v^{1}(0)=0, \quad\left(v^{1}\right)^{(1)}(0)=y_{x x}^{0}(0,0)=y_{0}^{(2)}(0)=M^{-2}\left(v^{0}\right)^{(2)}(0) \\
& \left(v^{1}\right)^{(2)}(0)=y_{x x t}^{0}(0,0)-M y_{x x x}^{0}=-2 M y_{0}^{(3)}(0)=2 M^{-2}\left(v^{0}\right)^{(3)}(0) \\
& \left(v^{1}\right)^{(3)}(0)=M^{2} y_{x x x x}^{0}-M y_{x x x t}^{0}(0,0)+y_{x x t t}^{0}(0,0)=3 M^{2} y^{(4)}(0)=3 M^{-2} v^{(4)}(0)
\end{aligned}
$$

while for $k=2$ we have

$$
v^{2}(0)=0, \quad\left(v^{2}\right)^{(1)}(0)=y_{x x}^{1}(0,0)=0 .
$$

Thus we retrieve the matching conditions (51) and (52).

Let us now establish the following result.

Lemma 4.7 Let $w_{m}^{\varepsilon}$ be the function defined by 60. Assume that the assumptions of Lemma 4.6 hold true. Then there is a constant $c_{m}$ independent of $\varepsilon$ such that

$$
\left\|L_{\varepsilon}\left(w_{m}^{\varepsilon}\right)\right\|_{C\left([0, T] ; L^{2}(0,1)\right)} \leq c_{m} \varepsilon^{\frac{(2 m+1) \gamma}{2}} .
$$

Proof. A straightforward calculation gives

$$
L_{\varepsilon}\left(w_{m}^{\varepsilon}\right)(x, t)=\sum_{i=1}^{5} J_{\varepsilon}^{i}(x, t),
$$


with

$$
\begin{aligned}
& J_{\varepsilon}^{1}(x, t)=-\varepsilon^{m+1} y_{x x}^{m}(x, t) \mathcal{X}_{\varepsilon}(x), \\
& J_{\varepsilon}^{2}(x, t)=\varepsilon^{m}\left(1-\mathcal{X}_{\varepsilon}(x)\right) Y_{t}^{m}\left(\frac{1-x}{\varepsilon}, t\right) \\
& J_{\varepsilon}^{3}(x, t)=M \mathcal{X}^{\prime}\left(\frac{1-x}{\varepsilon^{\gamma}}\right) \varepsilon^{-\gamma}\left(\sum_{k=0}^{m} \varepsilon^{k} Y^{k}\left(\frac{1-x}{\varepsilon}, t\right)-\sum_{k=0}^{m} \varepsilon^{k} y^{k}(x, t)\right), \\
& J_{\varepsilon}^{4}(x, t)=\mathcal{X}^{\prime \prime}\left(\frac{1-x}{\varepsilon^{\gamma}}\right) \varepsilon^{1-2 \gamma}\left(\sum_{k=0}^{m} \varepsilon^{k} Y^{k}\left(\frac{1-x}{\varepsilon}, t\right)-\sum_{k=0}^{m} \varepsilon^{k} y^{k}(x, t)\right), \\
& J_{\varepsilon}^{5}(x, t)=2 \mathcal{X}^{\prime}\left(\frac{1-x}{\varepsilon^{\gamma}}\right) \varepsilon^{1-\gamma}\left(\varepsilon^{-1} \sum_{k=0}^{m} \varepsilon^{k} Y_{z}^{k}\left(\frac{1-x}{\varepsilon}, t\right)+\sum_{k=0}^{m} \varepsilon^{k} y_{x}^{k}(x, t)\right) .
\end{aligned}
$$

Clearly,

$$
\left\|J_{\varepsilon}^{1}\right\|_{L^{\infty}\left(0, T ; L^{2}(0,1)\right)} \leq \varepsilon^{m+1}\left\|y_{x x}^{m}\right\|_{C\left([0, T] ; L^{2}(0,1)\right)} \leq c_{m} \varepsilon^{m+1},
$$

and

$$
\begin{aligned}
\left\|J_{\varepsilon}^{2}\right\|_{C\left([0, T] ; L^{2}(0,1)\right)} & \leq \varepsilon^{m}\left\|\left(1-\mathcal{X}_{\varepsilon}(x)\right) Y_{t}^{m}\left(\frac{1-x}{\varepsilon}, t\right)\right\|_{C\left([0, T] ; L^{2}(0,1)\right)} \\
& \leq \varepsilon^{m} \max _{t \in[0, T]}\left(\int_{1-2 \varepsilon^{\gamma}}^{1}\left|Y_{t}^{m}\left(\frac{1-x}{\varepsilon}, t\right)\right|^{2} d x\right)^{1 / 2} \leq \varepsilon^{m} \max _{t \in[0, T]}\left(\varepsilon \int_{0}^{\frac{2 \varepsilon \varepsilon^{\gamma}}{\varepsilon}}\left|Y_{t}^{m}(z)\right|^{2} d z\right)^{1 / 2} .
\end{aligned}
$$

Thanks to the explicit form 59 we have, for $0<\varepsilon \leq \varepsilon_{0}$ small enough,

$$
\begin{aligned}
\max _{t \in[0, T]}\left(\varepsilon \int_{0}^{\frac{2 \varepsilon \gamma}{\varepsilon}}\left|Y_{t}^{m}(z, t)\right|^{2} d z\right)^{1 / 2} & \leq c_{m}\left\|\frac{\partial^{m+1} y^{0}}{\partial x^{m} \partial t}\right\|_{C([0,1] \times[0, T])}\left(\varepsilon \int_{0}^{\frac{2 \varepsilon \gamma}{\varepsilon}} z^{2 m} d z\right)^{1 / 2} \\
& \leq c_{m} \varepsilon^{-m} \varepsilon^{\frac{2 m+1}{2} \gamma} .
\end{aligned}
$$

It results that $\left\|J_{\varepsilon}^{2}\right\|_{C\left([0, T] ; L^{2}(0,1)\right)} \leq c_{m} \varepsilon^{\frac{2 m+1}{2}} \gamma$. Using Taylor expansions, for $1-x=\varepsilon z \rightarrow 0$, we have

$$
\sum_{k=0}^{m} \varepsilon^{k} y^{k}(x, t)=\sum_{k=0}^{m} \varepsilon^{k} y^{k}(1-\varepsilon z, t)=\sum_{k=0}^{m} \varepsilon^{k}\left(\sum_{i=0}^{m-k} \frac{1}{i !} \frac{\partial^{i} y^{k}}{\partial x^{i}}(1, t)(-\varepsilon z)^{i}\right)+\varepsilon^{m} \mathcal{O}((\varepsilon z)) .
$$

According to 59 it results that

$$
\sum_{k=0}^{m} \varepsilon^{k} Y^{k}(z, t)-\sum_{k=0}^{m} \varepsilon^{k} y^{k}(1-\varepsilon z, t)=\varepsilon^{m} \mathcal{O}((\varepsilon z))+e^{-M z} \sum_{k=0}^{m} \varepsilon^{k} P^{k}(z, t) .
$$

Using the previous estimate we have

$$
\begin{aligned}
\left\|J_{\varepsilon}^{3}\right\|_{C\left([0, T] ; L^{2}(0,1)\right)} & =M \varepsilon^{-\gamma}\left\|\mathcal{X}^{\prime}\left(\frac{1-x}{\varepsilon^{\gamma}}\right)\left(\sum_{k=0}^{m} \varepsilon^{k} Y^{k}(z, t)-\sum_{k=0}^{m} \varepsilon^{k} y^{k}(1-\varepsilon z, t)\right)\right\|_{C\left([0, T] ; L^{2}(0,1)\right)} \\
& \leq c_{m} \varepsilon^{m-\gamma}\left(\int_{1-2 \varepsilon^{\gamma}}^{1-\varepsilon^{\gamma}}(1-x)^{2} d x\right)^{1 / 2} \leq c_{m} \varepsilon^{m+\frac{\gamma}{2}} .
\end{aligned}
$$

Similarly we have $\left\|J_{\varepsilon}^{4}\right\|_{C\left([0, T] ; L^{2}(0,1)\right)} \leq c_{m} \varepsilon^{(m+1)-\frac{\gamma}{2}}$. It results from 70 that

$$
\begin{aligned}
\varepsilon^{-1} \sum_{k=0}^{m} \varepsilon^{k} Y_{z}^{k}(z, t)+\sum_{k=0}^{m} \varepsilon^{k} y_{x}^{k}(1-\varepsilon z, t)= & \varepsilon^{m-1} \mathcal{O}((\varepsilon z))+\varepsilon^{-1} e^{-M z} \sum_{k=0}^{m} \varepsilon^{k} P_{z}^{k}(z, t) \\
& -\varepsilon^{-1} M e^{-M z} \sum_{k=0}^{m} \varepsilon^{k} P^{k}(z, t) .
\end{aligned}
$$


Arguing as for $J_{\varepsilon}^{3}$ we deduce that $\left\|J_{\varepsilon}^{5}\right\|_{C\left([0, T] ; L^{2}(0,1)\right)} \leq c_{m} \varepsilon^{m+\frac{\gamma}{2}}$. Collecting the previous estimates leads to 68 .

We now define the initial layer corrector $\theta_{m}^{\varepsilon}$ as the solution of

$$
\left\{\begin{array}{lr}
\theta_{m t}^{\varepsilon}-\varepsilon \theta_{m x x}^{\varepsilon}+M \theta_{m x}^{\varepsilon}=0, & (x, t) \in Q_{T}, \\
\theta_{m}^{\varepsilon}(0, t)=\theta_{m}^{\varepsilon}(1, t)=0, & t \in(0, T), \\
\theta_{m}^{\varepsilon}(x, 0)=\theta_{m 0}^{\varepsilon}(x), & x \in(0,1),
\end{array}\right.
$$

with

$$
\theta_{m 0}^{\varepsilon}(x)=: y_{0}(x)-w_{m}^{\varepsilon}(x, 0)=\left(1-\mathcal{X}_{\varepsilon}(x)\right)\left(y_{0}(x)-\sum_{k=0}^{m} \varepsilon^{k} Y^{k}\left(\frac{1-x}{\varepsilon}, 0\right)\right), \quad x \in(0,1) .
$$

We have the analog of Lemma 4.3 . We refer to [1] for the proof.

Lemma 4.8 Let $\theta_{m}^{\varepsilon}$ be the solution of problem (71), (72). Assume $\gamma \in(0,1 / 2]$. Then there exists a constant $c_{m}$, independent of $\varepsilon$, such that

$$
\left\|\theta_{m}^{\varepsilon}(\cdot, t)\right\|_{L^{2}(0,1)} \leq c_{m}\left(\varepsilon^{\frac{1}{2}}+\varepsilon^{\frac{(2 m+3) \gamma}{2}}\right) e^{-\frac{M^{2}}{2 \varepsilon^{\gamma}} t}, \quad \forall t \in[0, T]
$$

Arguing as in Section 4.1 .2 one can establish the analog of Lemma 4.4.

Lemma 4.9 Let $y^{\varepsilon}$ be the solution of problem (34), let $w_{m}^{\varepsilon}$ be the function defined by 60) and thet $\theta_{m}^{\varepsilon}$ be the solution of problem (71), 72. Assume that the assumptions of Lemma 4.1 hold true. Then there is a constant $c_{m}$, independent of $\varepsilon$, such that

$$
\left\|y^{\varepsilon}-w_{m}^{\varepsilon}-\theta_{m}^{\varepsilon}\right\|_{C\left([0, T] ; L^{2}(0,1)\right)} \leq c_{m} \varepsilon^{\frac{2 m+1}{2} \gamma} .
$$

Using Lemmas 4.8 and 4.9 we readily obtain the following result.

TheOREM 4.2 Let $y^{\varepsilon}$ be the solution of problem (34) and let $w_{m}^{\varepsilon}$ be the function defined by (60). Assume that the assumptions of Lemma 4.6 hold true and $\gamma \in(0,1 / 2]$. Then there exist two positive constants $c_{m}$ and $\varepsilon_{0}$, $c_{m}$ independent of $\varepsilon$, such that, for any $0<\varepsilon<\varepsilon_{0}$,

$$
\left\|y^{\varepsilon}(\cdot, t)-w_{m}^{\varepsilon}(\cdot, t)\right\|_{L^{2}(0,1)} \leq c_{m} \varepsilon^{\frac{2 m+1}{2} \gamma}+c_{m}\left(\varepsilon^{\frac{1}{2}}+\varepsilon^{\frac{(2 m+3) \gamma}{2}}\right) e^{-\frac{M^{2}}{2 \varepsilon \gamma} t}, \quad \forall t \in[0, T] .
$$

We have thus constructed a regular and strongly convergent approximation (as $\varepsilon \rightarrow 0) w_{m}^{\varepsilon}$ of $y^{\varepsilon}$, unique solution of 34 .

\subsubsection{Passing to the limit as $m \rightarrow \infty$. Particular case}

Our objective here is to show that, under some conditions on the initial condition $y_{0}$ and the functions $v^{k}$, we can pass to the limit with respect to the parameter $m$ and establish a convergence result of the sequence $\left(w_{m}^{\varepsilon}\right)_{m}$. We make the following assumptions:

(i) The initial condition $y_{0}$ belongs to $C^{\infty}[0,1]$ and there is $b \in \mathbb{R}$ such that

$$
\left\|y_{0}^{(k)}\right\|_{L^{2}(0,1)} \leq\left\lfloor\frac{k}{2}\right\rfloor ! b^{\frac{k}{2}}, \quad \forall k \in \mathbb{N},
$$

where $\lfloor$.$\rfloor denotes the integer part.$

(ii) $\left(v^{k}\right)_{k \geq 0}$ is a sequence of polynomials of degree $\leq p-1, p \geq 1$, uniformly bounded in $C^{p-1}[0, T]$. 
(iii) For any $k \in \mathbb{N}$, for any $m \in \mathbb{N}$, the functions $v^{k}$ and $y_{0}$ satisfy the matching conditions of Lemma 4.6 .

We establish the following result.

THEOREM 4.3 Let, for any $m \in \mathbb{N}, y_{m}^{\varepsilon}$ denote the solution of problem (34), and $w_{m}^{\varepsilon}$ the function defined by (60). We assume that the assumptions (i)-(iii) hold true and $\gamma \in(0,1 / 2]$. Then, there exist $\varepsilon_{0}>0$ and a function $\tilde{\theta}^{\varepsilon} \in L^{2}\left(0, T ; H_{0}^{1}(0,1)\right) \cap C\left([0, T] ; L^{2}(0,1)\right)$ satisfying an exponential decay, such that, for any fixed $0<\varepsilon<\varepsilon_{0}$, we have

$$
y_{m}^{\varepsilon}-w_{m}^{\varepsilon}-\tilde{\theta}^{\varepsilon} \rightarrow 0 \quad \text { in } C\left([0, T] ; L^{2}(0,1)\right), \quad \text { as } m \rightarrow+\infty .
$$

Consequently

$$
\begin{aligned}
\lim _{m \rightarrow+\infty} w_{m}^{\varepsilon}(x, t) & =\mathcal{X}_{\varepsilon}(x) \sum_{k=0}^{\infty} \varepsilon^{k} y^{k}(x, t)+\left(1-\mathcal{X}_{\varepsilon}(x)\right) \sum_{k=0}^{\infty} \varepsilon^{k} Y^{k}\left(\frac{1-x}{\varepsilon}, t\right)+\tilde{\theta}^{\varepsilon}(x, t) \\
& =y^{\varepsilon}(x, t)+\tilde{\theta}^{\varepsilon}(x, t) \quad \text { a.e. in } Q_{T},
\end{aligned}
$$

where $y^{\varepsilon}$ is the solution of problem (34) with $(34)_{2}$ replaced by $y^{\varepsilon}(0, t)=\sum_{k=0}^{\infty} \varepsilon^{k} v^{k}(t), \quad t \in(0, T)$. The function $\tilde{\theta}^{\varepsilon}$ satisfies

$$
\left\|\tilde{\theta}^{\varepsilon}(\cdot, t)\right\|_{L^{2}(0,1)} \leq c \varepsilon^{\frac{1}{2}} e^{-\frac{M^{2}}{2 \varepsilon \gamma} t}, \quad \forall t \in[0, T],
$$

where $c$ is a constant independent of $\varepsilon$.

The proof of this result requires the following lemma proved in [1].

Lemma 4.10 For $x<M t$, the function $y^{m}$ given by (37), (38) may be written in the form

$$
y^{m}(x, t)=v^{m}\left(t-\frac{x}{M}\right)+\sum_{j=1}^{m} \sum_{i=1}^{j} X_{j}^{i}(x)\left(v^{m-j}\right)^{(i+j)}\left(t-\frac{x}{M}\right),
$$

where, for any $1 \leq i \leq j \leq m, X_{j}^{i}(x)$ is a polynomial of degree $\leq i$.

Proof (of Theorem 4.3). Recall that (see 69) $L_{\varepsilon}\left(w_{m}^{\varepsilon}\right)(x, t)=\sum_{i=1}^{5} J_{\varepsilon}^{i}(x, t)$. We define

$$
f_{m}^{\varepsilon}=f_{m, 1}^{\varepsilon}+f_{m, 2}^{\varepsilon}+f_{m, 3}^{\varepsilon} \quad \text { in } Q_{T},
$$

with

$$
\begin{aligned}
& f_{m, 1}^{\varepsilon}(x, t)=-M \mathcal{X}^{\prime}\left(\frac{1-x}{\varepsilon^{\gamma}}\right) \varepsilon^{-\gamma} e^{-M \frac{1-x}{\varepsilon}} \sum_{k=0}^{m} \varepsilon^{k} P^{k}\left(\frac{1-x}{\varepsilon}, t\right) \\
& f_{m, 2}\left(x, t=-\mathcal{X}^{\prime \prime}\left(\frac{1-x}{\varepsilon^{\gamma}}\right) \varepsilon^{1-2 \gamma} e^{-M \frac{1-x}{\varepsilon}} \sum_{k=0}^{m} \varepsilon^{k} P^{k}\left(\frac{1-x}{\varepsilon}, t\right),\right. \\
& f_{m, 3}(x, t)=-2 \mathcal{X}^{\prime}\left(\frac{1-x}{\varepsilon^{\gamma}}\right) \varepsilon^{-\gamma} e^{-M \frac{1-x}{\varepsilon}}\left(\sum_{k=0}^{m} \varepsilon^{k} P_{z}^{k}\left(\frac{1-x}{\varepsilon}, t\right)-M \sum_{k=0}^{m} \varepsilon^{k} P^{k}\left(\frac{1-x}{\varepsilon}, t\right)\right) .
\end{aligned}
$$

We also define

$$
\tilde{\theta}_{m 0}^{\varepsilon}(x)=\left(1-\mathcal{X}_{\varepsilon}(x)\right)\left(y_{0}(x)-\sum_{k=0}^{m} \varepsilon^{k} Y^{k}\left(\frac{1-x}{\varepsilon}, 0\right)\right), \quad x \in(0,1) .
$$

Let $\tilde{\theta}_{m}^{\varepsilon}$ be the solution of the problem

$$
\left\{\begin{array}{lc}
L_{\varepsilon}\left(\tilde{\theta}_{m}^{\varepsilon}\right)=f_{m}^{\varepsilon}, & (x, t) \in Q_{T}, \\
\tilde{\theta}_{m}^{\varepsilon}(0, t)=\tilde{\theta}_{m}^{\varepsilon}(1, t)=0, & t \in(0, T), \\
\tilde{\theta}_{m}^{\varepsilon}(x, 0)=\tilde{\theta}_{m 0}^{\varepsilon}(x), & x \in(0,1),
\end{array}\right.
$$


so that the function $z_{m}^{\varepsilon}=: y_{m}^{\varepsilon}-w_{m}^{\varepsilon}-\tilde{\theta}_{m}^{\varepsilon}$ satisfies

$$
\left\{\begin{array}{lc}
L_{\varepsilon}\left(z_{m}^{\varepsilon}\right)=-L_{\varepsilon}\left(w_{m}^{\varepsilon}\right)-f_{m}^{\varepsilon}, & (x, t) \in Q_{T}, \\
z_{m}^{\varepsilon}(0, t)=z_{m}^{\varepsilon}(1, t)=0, & t \in(0, T), \\
z_{m}^{\varepsilon}(x, 0)=0, & x \in(0,1),
\end{array}\right.
$$

and then

$$
\int_{0}^{1}\left|z_{m}^{\varepsilon}(x, t)\right|^{2} d x \leq d_{m}^{\varepsilon} e^{t}, \quad d_{m}^{\varepsilon}:=\int_{0}^{T} \int_{0}^{1}\left|L_{\varepsilon}\left(w_{m}^{\varepsilon}\right)(x, s)+f_{m}^{\varepsilon}(x, s)\right|^{2} d x d s .
$$

Let us verify that $\left(d_{m}^{\varepsilon}\right)_{m>0}$ tends to 0 , as $m \rightarrow \infty$. We note that

$$
L_{\varepsilon}\left(w_{m}^{\varepsilon}\right)+f_{m}^{\varepsilon}=J_{\varepsilon}^{1}+J_{\varepsilon}^{2}+\left(J_{\varepsilon}^{3}+f_{m, 1}^{\varepsilon}\right)+\left(J_{\varepsilon}^{4}+f_{m, 2}^{\varepsilon}\right)+\left(J_{\varepsilon}^{5}+f_{m, 3}^{\varepsilon}\right) .
$$

- Estimate of $\left\|J_{\varepsilon}^{1}\right\|_{L^{2}\left(Q_{T}\right)}$ - It is easily seen that

$$
y_{x x}^{m}(x, t)=\frac{t^{m}}{m !} y_{0}^{(2 m+2)}(x-M t) \quad \text { for } x>M t .
$$

We set $Q_{T}^{-}=\left\{(x, t) \in Q_{T}: x>M t\right\}$ and $Q_{T}^{+}=\left\{(x, t) \in Q_{T}: x<M t\right\}$. Using $73 k k=2(m+1)$ we have

$$
\begin{aligned}
\int_{Q_{T}^{-}}\left|y_{x x}^{m}(x, t)\right|^{2} d x d t & =\int_{0}^{T} \frac{t^{2 m}}{(m !)^{2}} \int_{0}^{1-M t}\left|y_{0}^{(2 m+2)}(\xi)\right|^{2} d \xi d t \leq \frac{T^{2 m+1}}{(2 m+1)(m !)^{2}} \int_{0}^{1}\left|y_{0}^{(2 m+2)}(\xi)\right|^{2} d \xi \\
& \leq \frac{T^{2 m+1}}{(2 m+1)(m !)^{2}}((m+1) !)^{2} b^{2 m+2} \leq b(m+1)(T b)^{2 m+1} \leq b(\sqrt{2} T b)^{2 m+1}
\end{aligned}
$$

where we used the inequality $m+1 \leq 2^{m}$. For $x<M t$, it results from (74) that $y^{m}$ is a polynomial of degree $\leq p-1$ and, for large $m(m>p)$,

$$
y^{m}(x, t)=v^{m}\left(t-\frac{x}{M}\right)+\sum_{j=1}^{p-1} \sum_{i=1}^{j} X_{j}^{i}(x)\left(v^{m-j}\right)^{(i+j)}\left(t-\frac{x}{M}\right) .
$$

Since all the terms in the right-hand side of the previous inequality are uniformly bounded in the space $C^{p-1}\left(\overline{Q_{T}^{+}}\right)$, we deduce that there is a constant $c_{1}(p)$ independent of $m$ such that

$$
\max _{x \leq M t}\left|y_{x x}^{m}(x, t)\right|^{2} \leq c_{1}(p)
$$

then

$$
\int_{Q_{T}^{+}}\left|y_{x x}^{m}(x, t)\right|^{2} d x d t \leq c_{1}(p) T
$$

It results from 77 and 78 that

$$
\left\|J_{\varepsilon}^{1}\right\|_{L^{2}\left(Q_{T}\right)}^{2} \leq \varepsilon b(\sqrt{2} T b \varepsilon)^{2 m+1}+c_{1}(p) T \varepsilon^{2 m+2} .
$$

- Estimate of $\left\|J_{\varepsilon}^{2}\right\|_{L^{2}\left(Q_{T}\right)}$ - We have (see the proof of Lemma 4.7 )

$$
\left\|J_{\varepsilon}^{2}\right\|_{L^{2}\left(Q_{T}\right)}^{2} \leq \varepsilon^{2 m+1} \int_{0}^{T} \int_{0}^{\frac{2 \varepsilon^{\gamma}}{\varepsilon}}\left|Y_{t}^{m}(z, t)\right|^{2} d z d t .
$$

Thanks to Lemma 4.5 we have

$$
Y^{m}(z, t)=Q^{m}(z, t)+e^{-M z} P^{m}(z, t), \quad(z, t) \in(0,+\infty) \times(0, t),
$$


where

$$
P^{m}(z, t)=-\sum_{i=0}^{m} \frac{1}{i !} \frac{\partial^{i} y^{m-i}}{\partial x^{i}}(1, t) z^{i}, \quad Q^{m}(z, t)=\sum_{i=0}^{m} \frac{(-1)^{i}}{i !} \frac{\partial^{i} y^{m-i}}{\partial x^{i}}(1, t) z^{i}
$$

Then

$$
\int_{0}^{T} \int_{0}^{\frac{2 \varepsilon \gamma}{\varepsilon}}\left|Y_{t}^{m}(z, t)\right|^{2} d z d t \leq 2 \int_{0}^{T} \int_{0}^{\frac{2 \varepsilon \gamma}{\varepsilon}}\left(\left|Q_{t}^{m}(z, t)\right|^{2}+\left|P_{t}^{m}(z, t)\right|^{2}\right) d z d t .
$$

We have

$$
\begin{aligned}
\int_{0}^{T} \int_{0}^{\frac{2 \varepsilon \gamma}{\varepsilon}}\left|Q_{t}^{m}(z, t)\right|^{2} d z d t & \leq \int_{0}^{T} \int_{0}^{\frac{2 \varepsilon \gamma}{\varepsilon}}\left(\sum_{i=0}^{m} \frac{1}{i !}\left|\frac{\partial^{i+1} y^{m-i}}{\partial x^{i} \partial t}(1, t)\right| z^{i}\right)^{2} d z d t \\
& \leq(m+1) \sum_{i=0}^{m} \frac{1}{(i !)^{2}} \int_{0}^{T}\left|\frac{\partial^{i+1} y^{m-i}}{\partial x^{i} \partial t}(1, t)\right|^{2} d t \int_{0}^{\frac{2 \varepsilon}{\varepsilon}} z^{2 i} d z
\end{aligned}
$$

First, we easily verify that there is $\varepsilon_{1}>0$ such that, for $0<\varepsilon<\varepsilon_{1}$, we have

$$
q_{i}:=\int_{0}^{\frac{2 \varepsilon^{\gamma}}{\varepsilon}} z^{2 i} d z \leq q_{m}=\int_{0}^{\frac{2 \varepsilon^{\gamma}}{\varepsilon}} z^{2 m} d z=\frac{2^{2 m+1}}{2 m+1} \frac{\varepsilon^{(2 m+1) \gamma}}{\varepsilon^{2 m+1}}, \quad i=0, \ldots, m .
$$

Moreover, for $1<M t$, writing $y^{m-i}(x, t)$ in the form 74 , we deduce that there is a constant $c_{2}(p)$ independent of $m$ such that

$$
\max _{x \leq M t}\left|\frac{\partial^{i+1} y^{m-i}}{\partial x^{i} \partial t}(1, t)\right|^{2} \leq c_{2}(p) .
$$

On the other hand, for $1>M t$, we have

$$
\frac{\partial^{i+1} y^{m-i}}{\partial x^{i} \partial t}(1, t)=-M \frac{t^{m-i}}{(m-i) !} y_{0}^{(2 m-i+1)}(1-M t)+g_{i} \frac{t^{m-i-1}}{(m-i-1) !} y_{0}^{(2 m-i)}(1-M t), \quad 0 \leq i \leq m,
$$

with $g_{i}=1$ if $i<m$ and $g_{m}=0$. Consequently, for all $0 \leq i \leq m$,

$$
\left|\frac{\partial^{i+1} y^{m-i}}{\partial x^{i} \partial t}(1, t)\right|^{2} \leq 2 \frac{M^{2} t^{2 m-2 i}}{((m-i) !)^{2}}\left|y_{0}^{(2 m-i+1)}(1-M t)\right|^{2}+2 g_{i} \frac{t^{2 m-2 i-2}}{((m-i-1) !)^{2}}\left|y_{0}^{(2 m-i)}(1-M t)\right|^{2} .
$$

We set $B_{T}^{-}=\left\{(z, t) \in(0,+\infty) \times(0, T): 1>M t, 0<z<\frac{2 \varepsilon^{\gamma}}{\varepsilon}\right\}$ and $B_{T}^{+}=\{(z, t) \in(0,+\infty) \times(0, T): 1<$ $\left.M t, 0<z<\frac{2 \varepsilon^{\gamma}}{\varepsilon}\right\}$. We have

$$
\begin{aligned}
\int_{B_{T}^{-}}\left|Q_{t}^{m}(z, t)\right|^{2} d z d t & \left.\leq 2(m+1) M^{2} \sum_{i=0}^{m} \frac{1}{(i !)^{2}} \frac{T^{2 m-2 i} b^{2 m-i+1}}{((m-i) !)^{2}}\left(\mid m-\frac{i}{2}+\frac{1}{2}\right\rfloor !\right)^{2} q_{i} \\
& +2(m+1) \sum_{i=0}^{m-1} \frac{1}{(i !)^{2}} \frac{T^{2 m-2 i-2} b^{2 m-i}}{((m-i-1) !)^{2}}\left(\left\lfloor m-\frac{i}{2}\right\rfloor !\right)^{2} q_{i} \\
& \leq 2(m+1) M^{2} \bar{T}^{2 m} \bar{b}^{2 m+1} q_{m} \sum_{i=0}^{m} a_{m i}^{2}+2(m+1) \bar{T}^{2 m-2} \bar{b}^{2 m} q_{m} \sum_{i=0}^{m-1} b_{m i}^{2} \\
& \leq 2(m+1) q_{m} \bar{T}^{2 m-2} \bar{b}^{2 m}\left(M^{2} \bar{b} \bar{T}^{2} \sum_{i=0}^{m} a_{m i}^{2}+\sum_{i=0}^{m-1} b_{m i}^{2}\right)
\end{aligned}
$$

with $\bar{T}:=\max (1, T)$ and $\bar{b}:=\max (1, b)$ and $a_{m i}:=\frac{\left\lfloor m-\frac{i}{2}+\frac{1}{2}\right\rfloor !}{(m-i) ! i !}, b_{m i}:=\frac{\left\lfloor m-\frac{i}{2}\right\rfloor !}{(m-1) ! i !}$. Finally, we write

$$
\sum_{i=0}^{m} a_{m i}^{2} \leq\left(\sum_{i=0}^{m} a_{m i}\right)^{2} \leq(m+1)^{2} \sum_{i=0}^{m}\left(\frac{m !}{i !(m-i) !}\right)^{2}=(m+1)^{2}\left(2^{m}\right)^{2},
$$


in view of the binomial formulae. Similarly, $\sum_{i=0}^{m-1} b_{m i}^{2} \leq m^{2}\left(2^{m-1}\right)^{2}$. It results that

$$
\int_{B_{T}^{-}}\left|Q_{t}^{m}(z, t)\right|^{2} d z d t \leq 2(m+1) \frac{2^{2 m+1}}{2 m+1} \frac{\varepsilon^{(2 m+1) \gamma}}{\varepsilon^{2 m+1}} \bar{T}^{2 m-2} \bar{b}^{2 m}\left(M^{2} \bar{b} \bar{T}^{2}(m+1)^{2} 2^{2 m}+m^{2} 2^{2(m-1)}\right)
$$

and

$$
\begin{aligned}
\varepsilon^{2 m+1} \int_{B_{T}^{-}}\left|Q_{t}^{m}(z, t)\right|^{2} d z d t & \leq 2(m+1) \frac{2^{2 m+1}}{2 m+1} \varepsilon^{(2 m+1) \gamma} \bar{T}^{2 m-2} \bar{b}^{2 m}\left(M^{2} \bar{b}^{2}(m+1)^{2} 2^{2 m}+m^{2} 2^{2(m-1)}\right) \\
& \leq \frac{2(m+1)^{3}}{2 m+1}\left(1+M^{2} \bar{b} \bar{T}^{2}\right)\left(\bar{T} \bar{b} 4 \varepsilon^{\gamma}\right)^{2 m+1}
\end{aligned}
$$

which tends to 0 as $m \rightarrow \infty$ if $\varepsilon$ is small enough so that $\bar{T} \bar{b} 4 \varepsilon^{\gamma}<1$. The estimation for $\int_{B_{T}^{-}}\left|P_{t}^{m}(z, t)\right|^{2} d z d t$ is the same. On the other hand, a direct calculation shows that

$$
\left\|J_{\varepsilon}^{2}\right\|_{L^{2}\left(B_{T}^{+}\right)}^{2} \leq 4 c_{2}(p) e T \varepsilon^{2(m-p)}\left(2 \varepsilon^{\gamma}\right)^{2 p+1}
$$

Consequently,

$$
\left\|J_{\varepsilon}^{2}\right\|_{L^{2}\left(Q_{T}\right)}^{2} \leq \frac{8(m+1)^{3}}{2 m+1}\left(1+M^{2} \bar{b} \bar{T}^{2}\right)\left(\bar{T} \bar{b} 4 \varepsilon^{\gamma}\right)^{2 m+1}+4 c_{2}(p) e T \varepsilon^{2(m-p)}\left(2 \varepsilon^{\gamma}\right)^{2 p+1} .
$$

- Estimate of $\left\|J_{\varepsilon}^{3}+f_{m, 1}^{\varepsilon}\right\|_{L^{2}\left(Q_{T}\right)}$ - Using Taylor expansions, for $1-x=\varepsilon z \rightarrow 0$, we have

$$
\sum_{k=0}^{m} \varepsilon^{k} y^{k}(x, t)=\sum_{k=0}^{m} \varepsilon^{k} y^{k}(1-\varepsilon z, t)=\sum_{k=0}^{m} \varepsilon^{k}\left(\sum_{i=0}^{m-k} \frac{1}{i !} \frac{\partial^{i} y^{k}}{\partial x^{i}}(1, t)(-\varepsilon z)^{i}\right)+R_{m}^{\varepsilon}(z, t),
$$

with

$$
R_{m}^{\varepsilon}(z, t)=-\sum_{k=0}^{m} \varepsilon^{k} \int_{1-\varepsilon z}^{1} \frac{(1-\varepsilon z-s)^{m-k}}{(m-k) !} \frac{\partial^{m-k+1} y^{k}}{\partial x^{m-k+1}}(s, t) d s
$$

Then we have

$$
\sum_{k=0}^{m} \varepsilon^{k} Y^{k}\left(\frac{1-x}{\varepsilon}, t\right)-\sum_{k=0}^{m} \varepsilon^{k} y^{k}(x, t)=-R_{m}^{\varepsilon}(z, t)+e^{-M z} \sum_{k=0}^{m} \varepsilon^{k} P^{k}(z, t) .
$$

We deduce that

$$
\begin{aligned}
\left\|J_{\varepsilon}^{3}+f_{m, 1}^{\varepsilon}\right\|_{L^{2}\left(Q_{T}\right)}^{2} & =M^{2} \varepsilon^{-2 \gamma}\left\|\mathcal{X}^{\prime}\left(\frac{1-x}{\varepsilon^{\gamma}}\right)\left(\sum_{k=0}^{m} \varepsilon^{k} Q^{k}(z, t)-\sum_{k=0}^{m} \varepsilon^{k} y^{k}(1-\varepsilon z, t)\right)\right\|_{L^{2}\left(Q_{T}\right)}^{2} \\
& \leq M^{2} \varepsilon^{1-2 \gamma} \int_{0}^{T} \int_{\frac{\varepsilon \gamma}{\varepsilon}}^{\frac{2 \varepsilon \gamma}{\varepsilon}}\left|R_{m}^{\varepsilon}(z, t)\right|^{2} d z d t .
\end{aligned}
$$

We set $\tilde{B}_{T}^{-}=\left\{(z, t) \in(0,+\infty) \times(0, T): 1>M t, \frac{\varepsilon^{\gamma}}{\varepsilon}<z<\frac{2 \varepsilon^{\gamma}}{\varepsilon}\right\}$ and $\tilde{B}_{T}^{+}=\{(z, t) \in(0,+\infty) \times(0, T): 1<$ $\left.M t, \frac{\varepsilon^{\gamma}}{\varepsilon}<z<\frac{2 \varepsilon^{\gamma}}{\varepsilon}\right\}$. We have, for $1>M t, 1-\varepsilon z<s<1$,

$$
\frac{\partial^{m-k+1} y^{k}}{\partial x^{m-k+1}}(s, t)=\frac{t^{k}}{k !} y_{0}^{(k+m+1)}(s-M t) .
$$

Thanks to the continuous Sobolev embedding $H^{1}(0,1) \hookrightarrow C[0,1]$ there is a constant $c_{0}$ independent of $m$ such that

$$
\left\|y_{0}^{(k+m+1)}\right\|_{C[0,1]} \leq c_{0}\left(\left\|y_{0}^{(k+m+1)}\right\|_{L^{2}(0,1)}+\left\|y_{0}^{(k+m+2)}\right\|_{L^{2}(0,1)}\right) .
$$


Using 73 we then have

$$
\begin{aligned}
\left\|y_{0}^{(k+m+1)}\right\|_{C[0,1]} & \leq c_{0}\left(\left\lfloor\frac{k+m+1}{2}\right\rfloor !+\left\lfloor\frac{k+m+2}{2}\right\rfloor ! b^{\frac{1}{2}}\right) b^{\frac{k+m+1}{2}} \\
& \leq 2 c_{0} \sqrt{\bar{b}}\left\lfloor\frac{k+m+2}{2}\right\rfloor ! b^{\frac{k+m+1}{2}} .
\end{aligned}
$$

It results that, for $1>M t, 1-\varepsilon z<s<1$,

$$
\left|\frac{\partial^{m-k+1} y^{k}}{\partial x^{m-k+1}}(s, t)\right| \leq 2 c_{0} \sqrt{\bar{b}} \frac{t^{k}}{k !}\left\lfloor\frac{k+m+2}{2}\right\rfloor ! b^{\frac{k+m+1}{2}} .
$$

Therefore, for $(z, t) \in \tilde{B}_{T}^{-}$we have

$$
\begin{aligned}
\left|R_{m}^{\varepsilon}(z, t)\right| & \leq \sum_{k=0}^{m} \varepsilon^{k} \int_{1-\varepsilon z}^{1} \frac{(s-1+\varepsilon z)^{m-k}}{(m-k) !}\left|\frac{\partial^{m-k+1} y^{k}}{\partial x^{m-k+1}}(s, t)\right| d s \\
& \leq 2 c_{0} \sqrt{\bar{b}} \sum_{k=0}^{m} \varepsilon^{k} \frac{t^{k}}{k !}\left\lfloor\frac{k+m+2}{2}\right\rfloor ! b^{\frac{k+m+1}{2}} \int_{1-\varepsilon z}^{1} \frac{(s-1+\varepsilon z)^{m-k}}{(m-k) !} d s \\
& =2 c_{0} \sqrt{\bar{b}} \sum_{k=0}^{m} \varepsilon^{k} \frac{t^{k}}{k !}\left\lfloor\frac{k+m+2}{2}\right\rfloor ! b^{\frac{k+m+1}{2}} \frac{(\varepsilon z)^{m+1-k}}{(m+1-k) !} \\
& =2 c_{0} \sqrt{\bar{b}} \sum_{k=0}^{m} \varepsilon^{k} t^{k} \tilde{a}_{m k} b^{\frac{k+m+1}{2}}(\varepsilon z)^{m+1-k},
\end{aligned}
$$

with

It then follows

$$
\tilde{a}_{m k}:=\frac{1}{k !(m+1-k) !}\left\lfloor\frac{k+m+2}{2}\right\rfloor ! \leq \frac{(m+1) !}{k !(m+1-k) !}
$$

$$
\begin{aligned}
\int_{\tilde{B}_{T}^{-}}\left|R_{m}^{\varepsilon}(z, t)\right|^{2} d z d t & \leq 4 c_{0}^{2} \bar{b} \int_{0}^{T} \int_{\frac{\varepsilon \gamma}{\varepsilon}}^{\frac{2 \varepsilon \gamma}{\varepsilon}}\left(\sum_{k=0}^{m} \varepsilon^{k} t^{k} \tilde{a}_{m k} b^{\frac{k+m+1}{2}}(\varepsilon z)^{m+1-k}\right)^{2} d z d t \\
& \leq 4 c_{0}^{2} \bar{b}(m+1) \varepsilon^{2 m+2} \int_{0}^{T} \int_{\frac{\varepsilon \gamma}{\varepsilon}}^{\frac{2 \varepsilon \gamma}{\varepsilon}} \sum_{k=0}^{m} t^{2 k} \tilde{a}_{m k}^{2} b^{k+m+1} z^{2 m+2-2 k} d z d t \\
& \leq 4 c_{0}^{2} \bar{b}(m+1) \varepsilon^{2 m+2} \int_{0}^{T} \int_{\frac{\varepsilon \gamma}{\varepsilon}}^{\frac{2 \varepsilon}{\varepsilon}} \sum_{k=0}^{m} t^{2 k} \tilde{a}_{m k}^{2} b^{k+m+1} z^{2 m+2} d z d t \\
& \leq 2 c_{0}^{2} \bar{b} \frac{\left(2 \varepsilon^{\gamma}\right)^{2 m+3}}{\varepsilon} \sum_{k=0}^{m} \frac{\tilde{a}_{m k}^{2}}{2 k+1} T^{2 k+1} b^{k+m+1} \\
& \leq 2 c_{0}^{2} \bar{b} \frac{\left(2 \varepsilon^{\gamma}\right)^{2 m+3}}{\varepsilon}(\bar{b} \bar{T})^{2 m+1} \sum_{k=0}^{m} \tilde{a}_{m k}^{2} \\
& \leq 2 c_{0}^{2} \bar{b} \frac{\left(2 \varepsilon^{\gamma}\right)^{2 m+3}}{\varepsilon}(\bar{b} \bar{T})^{2 m+1} 2^{2(m+1)} \\
& \leq 16 c_{0}^{2} \bar{b} \varepsilon^{2 \gamma-1}\left(4 \varepsilon^{\gamma} \bar{b} \bar{T}\right)^{2 m+1} .
\end{aligned}
$$

We deduce that $\left\|J_{\varepsilon}^{3}+f_{m, 1}^{\varepsilon}\right\|_{L^{2}\left(\tilde{B}_{T}^{-}\right)}^{2} \leq 16 M^{2} c_{0}^{2} \bar{b}\left(4 \varepsilon^{\gamma} \bar{b} \bar{T}\right)^{2 m+1}$. We also have, for $(z, t) \in \tilde{B}_{T}^{+}$,

$$
\begin{aligned}
\left|R_{m}^{\varepsilon}(z, t)\right| & \leq \sum_{k=0}^{m} \varepsilon^{k} \int_{1-\varepsilon z}^{1} \frac{(s-1+\varepsilon z)^{m-k}}{(m-k) !}\left|\frac{\partial^{m-k+1} y^{k}}{\partial x^{m-k+1}}(s, t)\right| d s \\
& \leq c_{3}(p) \sum_{k=m-p+1}^{m} \varepsilon^{k} \int_{1-\varepsilon z}^{1} \frac{(s-1+\varepsilon z)^{m-k}}{(m-k) !} d s \\
& =c_{3}(p) \varepsilon^{m+1} \sum_{k=m-p+1}^{m} \varepsilon^{k} \frac{z^{m+1-k}}{(m+1-k) !}
\end{aligned}
$$


where $c_{3}(p)$ is a constant independent of $m$. Then

$$
\begin{aligned}
\int_{\tilde{B}_{T}^{+}}\left|R_{m}^{\varepsilon}(z, t)\right|^{2} d z d t & \leq p T c_{3}(p)^{2} \varepsilon^{2 m+2} \int_{\frac{\varepsilon \gamma}{\varepsilon}}^{\frac{2 \varepsilon \gamma}{\varepsilon}} \sum_{k=m-p+1}^{m} \frac{z^{2 m+2-2 k}}{((m+1-k) !)^{2}} d z \\
& \leq p T c_{3}(p)^{2} \varepsilon^{2 m+2} \int_{\frac{\varepsilon \gamma}{\varepsilon}}^{\frac{2 \varepsilon \gamma}{\varepsilon}} \sum_{k=m-p+1}^{m} \frac{z^{2 p}}{((m+1-k) !)^{2}} d z \\
& \leq p T c_{3}(p)^{2} \varepsilon^{2(m-p)+1} \frac{\left(2 \varepsilon^{\gamma}\right)^{2 p+1}}{2 p+1} \sum_{k=m-p+1}^{m} \frac{1}{((m+1-k) !)^{2}} \\
& \leq c_{4}(p) T\left(2 \varepsilon^{\gamma}\right)^{2 p+1} \varepsilon^{2(m-p)+1},
\end{aligned}
$$

where $c_{4}(p)$ is a constant independent of $m$. We conclude that

$$
\left\|J_{\varepsilon}^{3}+f_{m, 1}^{\varepsilon}\right\|_{L^{2}\left(Q_{T}\right)}^{2} \leq 16 M^{2} c_{0}^{2} \bar{b}\left(4 \varepsilon^{\gamma} \bar{b} \bar{T}\right)^{2 m+1}+c_{4}(p) M^{2} T \varepsilon^{2(1-\gamma)}\left(2 \varepsilon^{\gamma}\right)^{2 p+1} \varepsilon^{2(m-p)} .
$$

Estimates for $\left\|J_{\varepsilon}^{4}+f_{m, 2}^{\varepsilon}\right\|_{L^{2}\left(Q_{T}\right)}$ and $\left\|J_{\varepsilon}^{5}+f_{m, 3}^{\varepsilon}\right\|_{L^{2}\left(Q_{T}\right)}$ are very similar and are not detailed.

It results from the estimates $(79),(80), 820$, that we can choose $\varepsilon_{0}>0$ such that, for any fixed $0<\varepsilon<\varepsilon_{0}$, $\left(d_{m}^{\varepsilon}\right)_{m>0}$ (see 76]) tends to 0 , as $m \rightarrow \infty$. Thanks to $76,,\left(z_{m}^{\varepsilon}\right)_{m>0}$ tends to 0 as well, as $m \rightarrow \infty$.

- Estimate for $\left(\tilde{\theta}_{m}^{\varepsilon}\right)_{m}$, solution of 75 - We have $\tilde{\theta}_{m 0}^{\varepsilon}=a_{m}^{\varepsilon}+b_{m}^{\varepsilon}$ with

$$
\begin{aligned}
& a_{m}^{\varepsilon}(x)=\left(1-\mathcal{X}_{\varepsilon}(x)\right)\left(y_{0}(x)-\sum_{i=0}^{m} \frac{(-1)^{i}}{i !} y_{0}^{(i)}(1)(\varepsilon z)^{i}\right) \\
& b_{m}^{\varepsilon}(x)=\left(1-\mathcal{X}_{\varepsilon}(x)\right) e^{-M z} \sum_{i=0}^{m} \frac{1}{i !} y_{0}^{(i)}(1)(\varepsilon z)^{i}, \quad\left(z=\frac{1-x}{\varepsilon}\right) .
\end{aligned}
$$

Using Taylor's expansion, for $1-x=\varepsilon z \rightarrow 0$, we have

$$
a_{m}^{\varepsilon}(x)=\left(1-\mathcal{X}_{\varepsilon}(x)\right) \frac{(1-x)^{m+1}}{(m+1) !} y_{0}^{(m+1)}\left(\zeta_{1}\right), \quad \zeta_{1} \in(x, 1),
$$

hence

$$
\left|a_{m}^{\varepsilon}(x)\right| \leq \frac{\left(2 \varepsilon^{\gamma}\right)^{m+1}}{(m+1) !}\left\|y_{0}^{(m+1)}\right\|_{C[0,1]}, \quad x \in\left(1-2 \varepsilon^{\gamma}, 1\right) .
$$

Using 81 with $k=0$, we obtain for all $x \in\left(1-2 \varepsilon^{\gamma}, 1\right)$ that

$$
\left|a_{m}^{\varepsilon}(x)\right| \leq 2 c_{0} \sqrt{\bar{b}} \frac{\left(2 \sqrt{b} \varepsilon^{\gamma}\right)^{m+1}}{(m+1) !}\left\lfloor\frac{m+2}{2}\right\rfloor ! \leq 2 c_{0} \sqrt{\bar{b}}\left(2 \sqrt{b} \varepsilon^{\gamma}\right)^{m+1} .
$$

We conclude that $\left(a_{m}^{\varepsilon}\right)$ converges uniformly in $[0,1]$ to 0 . Moreover, the series $\sum_{i=0}^{\infty} \frac{y_{0}^{(i)}(1)}{i !}(1-x)^{i}$ is uniformly convergent since in $\left[1-2 \varepsilon^{\gamma}, 1\right]$

$$
\sum_{i=0}^{m}\left|\frac{y_{0}^{(i)}(1)}{i !}(1-x)^{i}\right| \leq \sum_{i=0}^{\infty} \frac{\left(2 \varepsilon^{\gamma}\right)^{i}}{i !}\left\|y_{0}^{(i)}\right\|_{C[0,1]} \leq 2 c_{0} \sqrt{\bar{b}} \sum_{i=0}^{\infty} \frac{\left(2 \sqrt{b} \varepsilon^{\gamma}\right)^{i}}{i !}\left\lfloor\frac{i+1}{2}\right\rfloor ! \leq \frac{2 c_{0} \sqrt{\bar{b}}}{1-2 \sqrt{b} \varepsilon^{\gamma}}
$$

Here we have used again the inequality $\left\|y_{0}^{(i)}\right\|_{C[0,1]} \leq c_{0}\left(\left\|y_{0}^{(i)}\right\|_{\left.L^{2} 0,1\right)}+\left\|y_{0}^{(i+1)}\right\|_{\left.L^{2} 0,1\right)}\right)$ and $(73)$. Then $\left(\tilde{\theta}_{m 0}^{\varepsilon}\right)_{m}$ converges uniformly in $[0,1]$ to $\tilde{\theta}_{0}^{\varepsilon}$ given by

$$
\tilde{\theta}_{0}^{\varepsilon}(x)=\left(1-\mathcal{X}_{\varepsilon}(x)\right) e^{-M \frac{1-x}{\varepsilon}} \sum_{i=0}^{\infty} \frac{y_{0}^{(i)}(1)}{i !}(1-x)^{i}, \quad x \in(0,1) .
$$


Moreover, $\tilde{\theta}_{0}^{\varepsilon}$ satisfies an exponential decay property:

$$
\left|\tilde{\theta}_{0}^{\varepsilon}(x)\right| \leq \frac{2 c_{0} \sqrt{\bar{b}}}{1-2 \sqrt{b} \varepsilon^{\gamma}}\left(1-\mathcal{X}_{\varepsilon}(x)\right) e^{-M \frac{1-x}{\varepsilon}}, \quad \forall x \in(0,1) .
$$

Consider now the function $f_{m, 1}^{\varepsilon}$. We have

$$
\sum_{k=0}^{m} \varepsilon^{k}\left|P^{k}\left(\frac{1-x}{\varepsilon}, t\right)\right| \leq \sum_{k=0}^{m} \varepsilon^{k}\left(\sum_{i=0}^{k}\left|\frac{\partial^{i} y^{k-i}}{\partial x^{i}}(1, t)\right| \frac{z^{i}}{i !}\right), \quad(x, t) \in Q_{T}, z=\frac{1-x}{\varepsilon},
$$

then, for $1-2 \varepsilon^{\gamma}<x<1-\varepsilon^{\gamma}$, and $1>M t$, we have

$$
\sum_{k=0}^{m} \varepsilon^{k}\left|P^{k}\left(\frac{1-x}{\varepsilon}, t\right)\right| \leq \sum_{k=0}^{m} \varepsilon^{k}\left(\sum_{i=0}^{k} \frac{T^{k-i}\left|y_{0}^{(2 k-i)}(1-M t)\right|}{(k-i) !} \frac{z^{i}}{i !}\right) .
$$

Using the continuous Sobolev embedding and 73 we obtain again

$$
\left\|y_{0}^{(2 k-i)}\right\|_{C[0,1]} \leq 2 c_{0} \sqrt{\bar{b}}\left\lfloor\frac{2 k-i+1}{2}\right\rfloor ! b^{\frac{2 k-i}{2}} .
$$

Then

$$
\begin{aligned}
\sum_{k=0}^{m} \varepsilon^{k}\left|P^{k}\left(\frac{1-x}{\varepsilon}, t\right)\right| & \leq 2 c_{0} \sqrt{\bar{b}} \sum_{k=0}^{m} \varepsilon^{k}\left(\sum_{i=0}^{k} \frac{T^{k-i}\left\lfloor\frac{2 k-i+1}{2}\right\rfloor ! b^{\frac{2 k-i}{2}}}{(k-i) ! i !} z^{i}\right) \\
& \leq 2 c_{0} \sqrt{\bar{b}} \sum_{k=0}^{m}(\bar{T} \bar{b} \varepsilon)^{k} \frac{\left(2 \varepsilon^{\gamma}\right)^{k}}{\varepsilon^{k}} \sum_{i=0}^{k} a_{k i},
\end{aligned}
$$

using that $z^{i} \leq \frac{\left(2 \varepsilon^{\gamma}\right)^{k}}{\varepsilon^{k}}$, for all $0 \leq i \leq k$ and $a_{k i}:=\frac{\left\lfloor k-\frac{i}{2}+\frac{1}{2}\right\rfloor !}{(k-i) ! i !}$. Writing that $\sum_{i=0}^{k} a_{k i} \leq 2^{k}$, we finally obtain

$$
\sum_{k=0}^{m} \varepsilon^{k}\left|P^{k}\left(\frac{1-x}{\varepsilon}, t\right)\right| \leq 2 c_{0} \sqrt{\bar{b}} \sum_{k=0}^{m}\left(4 \bar{T} \bar{b} \varepsilon^{\gamma}\right)^{k} \leq 2 c_{0} \sqrt{\bar{b}} \frac{1-\left(4 \bar{T} \bar{b} \varepsilon^{\gamma}\right)^{m+1}}{1-4 \bar{T} \bar{b} \varepsilon^{\gamma}}
$$

assuming $\varepsilon$ small enough so that $1-4 \bar{T} \bar{b} \varepsilon^{\gamma}>0$. On the other hand, for $1-2 \varepsilon^{\gamma} \leq x \leq 1-\varepsilon^{\gamma}$ and $1<M t$, we write

$$
\begin{aligned}
\sum_{k=0}^{m} \varepsilon^{k}\left|P^{k}\left(\frac{1-x}{\varepsilon}, t\right)\right| & \leq \sum_{k=0}^{m} \varepsilon^{k}\left(\sum_{i=0}^{p}\left|\frac{\partial^{i} y^{k-i}}{\partial x^{i}}(1, t)\right| \frac{z^{i}}{i !}\right) \\
& \leq c_{5}(p)\left(\sum_{k=0}^{m} \varepsilon^{k}\right)\left(\sum_{i=0}^{p} \frac{z^{i}}{i !}\right) \\
& \leq \frac{c_{5}(p) e}{1-\varepsilon} \frac{\left(2 \varepsilon^{\gamma}\right)^{p}}{\varepsilon^{p}}
\end{aligned}
$$

where $c_{5}(p)$ is a constant independent of $m$. Then, for $0<\varepsilon^{\gamma}<1 /(4 \bar{T} \bar{b})$, the series $\sum_{k=0}^{\infty} \varepsilon^{k} P^{k}\left(\frac{1-x}{\varepsilon}, t\right)$ is uniformly convergent in $\overline{Q_{T}}$. This implies that $\left(f_{m, 1}^{\varepsilon}\right)_{m}$ converges uniformly in $\overline{Q_{T}}$ to a function $f_{1}^{\varepsilon}$ given by

$$
f_{1}^{\varepsilon}(x, t)=-M \mathcal{X}^{\prime}\left(\frac{1-x}{\varepsilon^{\gamma}}\right) \varepsilon^{-\gamma} e^{-M \frac{1-x}{\varepsilon}} \sum_{k=0}^{\infty} \varepsilon^{k} P^{k}\left(\frac{1-x}{\varepsilon}, t\right), \quad(x, t) \in Q_{T} .
$$

Moreover, $f_{1}^{\varepsilon}$ has an exponential decay property:

$$
\left|f_{1}^{\varepsilon}(x, t)\right| \leq M\left|\mathcal{X}^{\prime}\left(\frac{1-x}{\varepsilon^{\gamma}}\right)\right|\left(\frac{2 c_{0} \sqrt{\bar{b}}}{1-4 \bar{T} \bar{b} \varepsilon^{\gamma}}+\frac{c_{5}(p) e}{1-\varepsilon} \frac{\left(2 \varepsilon^{\gamma}\right)^{p}}{\varepsilon^{p}}\right) \times \varepsilon^{-\gamma} e^{-2 M \frac{\varepsilon^{\gamma}}{\varepsilon}},
$$


for $(x, t) \in Q_{T}$. Clearly, $\left(f_{m, 2}^{\varepsilon}\right)_{m}$ converges uniformly in $\overline{Q_{T}}$ to a function $f_{2}^{\varepsilon}$ satisfying a similar property of exponential decay. Similar arguments allow to show that $\left(f_{m, 3}^{\varepsilon}\right)_{m}$ converges uniformly in $\overline{Q_{T}}$ to a function $f_{3}^{\varepsilon}$ satisfying a property of exponential decay. Note also that the functions $f_{1}^{\varepsilon}, f_{2}^{\varepsilon}$ and $f_{3}^{\varepsilon}$ have supports contained in $\left[1-2 \varepsilon^{\gamma}, 1-\varepsilon^{\gamma}\right] \times[0, T]$. Thus $\left(f_{m}^{\varepsilon}\right)_{m}$ converges uniformly in $\overline{Q_{T}}$ to a function $f^{\varepsilon}=f_{1}^{\varepsilon}+f_{2}^{\varepsilon}+f_{3}^{\varepsilon}$ satisfying a property of exponential decay.

Let $\tilde{\theta}^{\varepsilon}$ be the solution of the problem

$$
\left\{\begin{array}{lc}
L_{\varepsilon}\left(\tilde{\theta}^{\varepsilon}\right)=f^{\varepsilon}, & \left.(x, t) \in Q_{T}\right), \\
\tilde{\theta}^{\varepsilon}(0, t)=\tilde{\theta}^{\varepsilon}(1, t)=0, & t \in(0, T), \\
\tilde{\theta}^{\varepsilon}(x, 0)=\tilde{\theta}_{0}^{\varepsilon}(x), & x \in(0,1) .
\end{array}\right.
$$

To show the exponential decay of $\tilde{\theta}^{\varepsilon}$ we proceed as in the proof of Lemma 4.3 We use notably, from the exponential decay of $f^{\varepsilon}$, that there is a constant $c_{0}$ such that

$$
\left|f^{\varepsilon}(x, t)\right| \leq c_{0} \varepsilon^{-\gamma} e^{-M \frac{\varepsilon \gamma}{\varepsilon}} \quad \text { in } Q_{T} .
$$

Then from 75 and $(84)$ we deduce that

$$
\left\|\tilde{\theta}_{m}^{\varepsilon}(\cdot, t)-\tilde{\theta}^{\varepsilon}(\cdot, t)\right\|_{L^{2}(0,1)}^{2} \leq\left(\left\|f_{m}^{\varepsilon}-f^{\varepsilon}\right\|_{L^{2}\left(Q_{T}\right)}^{2}+\left\|\tilde{\theta}_{m 0}^{\varepsilon}-\tilde{\theta}_{0}^{\varepsilon}\right\|_{L^{2}(0,1)}\right) e^{t}, \quad \forall t \in(0, T),
$$

which implies that $\left(\tilde{\theta}_{m}^{\varepsilon}\right)_{m}$ converges in $C\left([0, T] ; L^{2}(0,1)\right)$ to $\tilde{\theta}^{\varepsilon}$. This completes the proof of the theorem.

Remark 12 Polynomials satisfy assumption (i) of Theorem 4.3. In fact, the functions satisfying assumption (i) can be identified as the analytic functions of the Gevrey class of order $1 / 2$ on $[0,1]$, denoted $G^{1 / 2}[0,1]$ (see [25]). Recall that a function $f \in C^{\infty}[0,1]$ is said to belong to $G^{1 / 2}[0,1]$ if there exist two positive constants a and $\tilde{b}$ such

$$
\left\|f^{(k)}\right\|_{C([0,1])} \leq a(k !)^{\frac{1}{2}} \tilde{b}^{k}, \quad \forall k \in \mathbb{N} .
$$

Using the Stirling formula, one can verify that every function $f \in G^{1 / 2}[0,1]$ satisfies assumption (i). Conversely, using the continuous Sobolev embedding $H^{1}(0,1) \hookrightarrow C[0,1]$ and the Stirling formula, one can verify that every function satisfying assumption (i) belongs to $G^{1 / 2}[0,1]$.

\subsubsection{Asymptotic approximation of the adjoint solution $\varphi^{\varepsilon}$}

Let us consider the adjoint problem

$$
\left\{\begin{array}{lc}
-\varphi_{t}^{\varepsilon}-\varepsilon \varphi_{x x}^{\varepsilon}-M \varphi_{x}^{\varepsilon}=0, & (x, t) \in Q_{T}, \\
\varphi^{\varepsilon}(0, t)=\varphi^{\varepsilon}(1, t)=0, & t \in(0, T), \\
\varphi^{\varepsilon}(x, T)=\varphi_{T}^{\varepsilon}(x), & x \in(0,1),
\end{array}\right.
$$

where $\varphi_{T}^{\varepsilon}$ is a function of the form $\varphi_{T}^{\varepsilon}=\sum_{k=0}^{m} \varepsilon^{k} \varphi_{T}^{k}$, the functions $\varphi_{T}^{0}, \varphi_{T}^{1}, \cdots, \varphi_{T}^{m}$ being given. We assume $M>0$, the case $M<0$ can be treated similarly. We construct an asymptotic approximation of the solution $\varphi^{\varepsilon}$ of 85 by using the matched asymptotic expansion method.

To get the outer expansion $\sum_{k=0}^{m} \varepsilon^{k} \varphi^{k}(x, t)$ of $\varphi^{\varepsilon}$ we repeat again the procedure performed for the direct solution $y^{\varepsilon}$. From equation 85 we have

$$
\begin{array}{ll}
\varepsilon^{0}: & \varphi_{t}^{0}+M \varphi_{x}^{0}=0, \\
\varepsilon^{k}: & \varphi_{t}^{k}+M \varphi_{x}^{k}=-\varphi_{x x}^{k-1}, \quad 1 \leq k \leq m .
\end{array}
$$


Taking the initial and boundary conditions into account we define $\varphi^{0}$ and $\varphi^{k}(1 \leq k \leq m)$ as functions satisfying the transport equations, respectively,

$$
\left\{\begin{array}{lr}
\varphi_{t}^{0}+M \varphi_{x}^{0}=0, & (x, t) \in Q_{T}, \\
\varphi^{0}(1, t)=0, & t \in(0, T), \\
\varphi^{0}(x, T)=\varphi_{T}^{0}(x), & x \in(0,1),
\end{array}\right.
$$

and

$$
\left\{\begin{array}{lr}
\varphi_{t}^{k}+M \varphi_{x}^{k}=-\varphi_{x x}^{k-1}, & (x, t) \in Q_{T}, \\
\varphi^{k}(1, t)=0, & t \in(0, T), \\
\varphi^{k}(x, T)=\varphi_{T}^{k}(x), & x \in(0,1) .
\end{array}\right.
$$

The solution of $(86)$ is given by

$$
\varphi^{0}(x, t)= \begin{cases}0, & x>1+M(t-T), \\ \varphi_{T}^{0}(x+M(T-t)), & x<1+M(t-T) .\end{cases}
$$

Using the method of characteristics we find that, for any $1 \leq k \leq m$,

$$
\varphi^{k}(x, t)= \begin{cases}\int_{t}^{t+(1-x) / M} \varphi_{x x}^{k-1}(x+M(s-t), s) d s, & x>1+M(t-T), \\ \varphi_{T}^{k}(x+M(T-t))+\int_{t}^{T} \varphi_{x x}^{k-1}(x+M(s-t), s) d s, & x<1+M(t-T) .\end{cases}
$$

The inner expansion is given by

$$
\sum_{k=0}^{m} \varepsilon^{k} \Phi^{k}(z, t), \quad z=\frac{x}{\varepsilon} \in\left(0, \varepsilon^{-1}\right), t \in(0, T),
$$

with functions $\Phi^{0}$ and $\Phi^{k}(1 \leq k \leq m)$ satisfying the equations, respectively,

$$
\begin{aligned}
& \Phi_{z z}^{0}(z, t)+M \Phi_{z}^{0}(z, t)=0, \\
& \Phi_{z z}^{k}(z, t)+M \Phi_{z}^{k}(z, t)=-\Phi_{t}^{k}(z, t) .
\end{aligned}
$$

We define $\Phi^{0}$ as a solution of

$$
\left\{\begin{array}{lr}
\Phi_{z z}^{0}(z, t)+M \Phi_{z}^{0}(z, t)=0, & (z, t) \in(0,+\infty) \times(0, T) \\
\Phi^{0}(0, t)=0, & t \in(0, T) \\
\lim _{z \rightarrow+\infty} \Phi^{0}(z, t)=\lim _{x \rightarrow 0} \varphi^{0}(x, t), & t \in(0, T) .
\end{array}\right.
$$

The solution of 88 reads

$$
\Phi^{0}(z, t)=\varphi^{0}(0, t)\left(1-e^{-M z}\right), \quad(z, t) \in(0,+\infty) \times(0, T) .
$$

For $1 \leq k \leq m$, the function $\Phi^{k}$ is defined iteratively as a solution of

$$
\left\{\begin{array}{lr}
\Phi_{z z}^{k}(z, t)+M \Phi_{z}^{k}(z, t)=-\Phi_{t}^{k-1}(z, t), & (z, t) \in(0,+\infty) \times(0, T), \\
\Phi^{k}(0, t)=0, & t \in(0, T), \\
\lim _{z \rightarrow+\infty}\left[\Phi^{k}(z, t)-S^{k}(z, t)\right]=0, & t \in(0, T),
\end{array}\right.
$$

where

$$
S^{k}(z, t)=\sum_{i=0}^{k} \frac{1}{i !} \frac{\partial^{i} \varphi^{k-i}}{\partial x^{i}}(0, t) z^{i}
$$


Arguing as in Lemma 4.5 one can verify that the solution of problem 90 reads

$$
\Phi^{k}(z, t)=S^{k}(z, t)+e^{-M z} R^{k}(z, t), \quad(z, t) \in(0,+\infty) \times(0, t),
$$

where

$$
R^{k}(z, t)=\sum_{i=0}^{k} \frac{(-1)^{i+1}}{i !} \frac{\partial^{i} \varphi^{k-i}}{\partial x^{i}}(0, t) z^{i} .
$$

Let $\mathcal{X}: \mathbb{R} \rightarrow[0,1]$ denote a $C^{\infty}$ cut-off function satisfying [47). We define, for $\gamma \in(0,1)$, the function

$$
\mathcal{X}_{\varepsilon}(x)=\mathcal{X}\left(\frac{x}{\varepsilon^{\gamma}}\right)
$$

then introduce the function

$$
\psi_{m}^{\varepsilon}(x, t)=\mathcal{X}_{\varepsilon}(x) \sum_{k=0}^{m} \varepsilon^{k} \varphi^{k}(x, t)+\left(1-\mathcal{X}_{\varepsilon}(x)\right) \sum_{k=0}^{m} \varepsilon^{k} \Phi^{k}\left(\frac{x}{\varepsilon}, t\right),
$$

defined to be an asymptotic approximation at order $m$ of the solution $\varphi^{\varepsilon}$ of 85 . To justify all the computations we will perform we need some regularity assumptions on the data $\varphi^{0}$ and $\varphi^{k}, 1 \leq k \leq m$. We have the following result.

Lemma 4.11 Assume that, for any $0 \leq k \leq m, \varphi_{T}^{k} \in C^{2(m-k)+1}[0, T]$, and the following $C^{2(m-k)+1}$-matching conditions are satisfied, respectively,

$$
\left(\varphi_{T}^{k}\right)^{(p)}(1)=0, \quad 0 \leq p \leq 2(m-k)+1 .
$$

Then the function $\varphi^{k}$ belongs to $C^{2(m-k)+1}\left(\overline{Q_{T}}\right)$.

A straightforward calculation gives $L_{\varepsilon}^{\star}\left(\psi_{m}^{\varepsilon}\right)(x, t)=\sum_{i=1}^{5} E_{\varepsilon}^{i}(x, t)$, with

$$
\begin{aligned}
& E_{\varepsilon}^{1}(x, t)=-\varepsilon^{m+1} \varphi_{x x}^{m}(x, t) \mathcal{X}_{\varepsilon}(x), \\
& E_{\varepsilon}^{2}(x, t)=-\varepsilon^{m}\left(1-\mathcal{X}_{\varepsilon}(x)\right) \Phi_{t}^{m}\left(\frac{x}{\varepsilon}, t\right), \\
& E_{\varepsilon}^{3}(x, t)=M \mathcal{X}^{\prime}\left(\frac{x}{\varepsilon^{\gamma}}\right) \varepsilon^{-\gamma}\left(\sum_{k=0}^{m} \varepsilon^{k} \Phi^{k}\left(\frac{x}{\varepsilon}, t\right)-\sum_{k=0}^{m} \varepsilon^{k} \varphi^{k}(x, t)\right), \\
& E_{\varepsilon}^{4}(x, t)=\mathcal{X}^{\prime \prime}\left(\frac{x}{\varepsilon^{\gamma}}\right) \varepsilon^{1-2 \gamma}\left(\sum_{k=0}^{m} \varepsilon^{k} \Phi^{k}\left(\frac{x}{\varepsilon}, t\right)-\sum_{k=0}^{m} \varepsilon^{k} \varphi^{k}(x, t)\right), \\
& E_{\varepsilon}^{5}(x, t)=2 \mathcal{X}^{\prime}\left(\frac{x}{\varepsilon^{\gamma}}\right) \varepsilon^{1-\gamma}\left(\varepsilon^{-1} \sum_{k=0}^{m} \varepsilon^{k} \Phi_{z}^{k}\left(\frac{x}{\varepsilon}, t\right)-\sum_{k=0}^{m} \varepsilon^{k} \varphi_{x}^{k}(x, t)\right) .
\end{aligned}
$$

We have the analogue of Lemma 4.7 .

Lemma 4.12 Assume that the assumptions of Lemma 4.11 hold true. Let $\psi_{m}^{\varepsilon}$ be the function defined by 91. Then there is a constant $c_{m}$ independent of $\varepsilon$ such that

$$
\left\|L_{\varepsilon}^{\star}\left(\psi_{m}^{\varepsilon}\right)\right\|_{C\left([0, T] ; L^{2}(0,1)\right)} \leq c_{m} \varepsilon^{\frac{(2 m+1) \gamma}{2}} .
$$

Using Lemma 4.12 we can argue as in the proof of Theorem 4.1 to establish the following result.

THEOREM 4.4 Let $\varphi^{\varepsilon}$ be the solution of problem (85) and let $\psi_{m}^{\varepsilon}$ be the function defined by (91). Assume that the assumptions of Lemma 4.11 hold true and $\gamma \in(0,1 / 2]$. Then there is a constant $c_{m}$ independent of $\varepsilon$ such that

$$
\left\|\varphi^{\varepsilon}(\cdot, t)-\psi_{m}^{\varepsilon}(\cdot, t)\right\|_{L^{2}(0,1)} \leq c_{m} \varepsilon^{\frac{2 m+1}{2} \gamma}+c_{m}\left(\varepsilon^{\frac{1}{2}}+\varepsilon^{\frac{(2 m+3) \gamma}{2}}\right) e^{-\frac{M^{2}}{2 \varepsilon \gamma}(T-t)}, \quad \forall t \in[0, T] .
$$




\section{Applications to controllability property}

We may use the previous asymptotic analysis to state $\varepsilon$-approximate controllability results.

Proposition 5.1 Let $m \in \mathbb{N}, T>\frac{1}{M}$ and $\left.a \in\right] 0, T-\frac{1}{M}[$. Assume that the assumptions on the initial condition $y_{0}$ and functions $v^{k}, 0 \leq k \leq m$ of Lemma 4.6 hold true. Assume moreover that

$$
v^{k}(t)=0, \quad 0 \leq k \leq m, \forall t \in[a, T]
$$

Then, the solution $y^{\varepsilon}$ of problem (34) satisfies the following property

$$
\left\|y^{\varepsilon}(\cdot, T)\right\|_{L^{2}(0,1)} \leq c_{m} \varepsilon^{\frac{(2 m+1) \gamma}{2}}, \quad \forall \gamma \in(0,1)
$$

for some constant $c_{m}>0$ independent of $\varepsilon$.

In other words, the function $v^{\varepsilon} \in C([0, T])$ defined by $v^{\varepsilon}:=\sum_{k=0}^{m} \varepsilon^{k} v^{k}$ is an approximate null control for (1): for any $\eta>0$, there exists $\varepsilon_{0}$, such that for any $\varepsilon \in\left(0, \varepsilon_{0}\right),\left\|y^{\varepsilon}(\cdot, T)\right\|_{L^{2}(0,1)} \leq \eta$.

Proof. We first check by induction that the function $y^{k}, 0 \leq k \leq m$ given by (37) and (38) vanishes at time T. From (37) and the assumption $\left[92,, y^{0}(x, t)=0\right.$ on the set

$$
S_{a}:=\{(x, t) \in(0,1) \times(0, T), t M-x \geq a M\}
$$

which contains the set $S_{T-1 / M}$ and the set $\{0,1\} \times\{T\}$. Assume now that $y^{k-1}(x, t)=0$ on $S_{a}$, for some $k \geq 1$. 63) implies that, for all $(x, t) \in S_{a}$

$$
y^{k}(x, t)=v^{k}\left(t-\frac{x}{M}\right)+\int_{t-x / M}^{t} y_{x x}^{k-1}(x+M(s-t), s) d s
$$

From (92), the first term vanishes because $t-\frac{x}{M} \geq a$ for all $(x, t) \in S_{a}$. Moreover, for $(x, t) \in S_{a}$, the segment $[x+M(s-t), s]$ for $s \in[t-x / M, t] \subset[a, T]$ belongs to $S_{a}$. Consequently, the second term vanishes as well and $y^{k}(x, t)=0,0 \leq k \leq m$ for all $(x, t) \in S_{a}$. In particular $y^{k}(x, T)=0,0 \leq k \leq m$ for all $x \in[0,1]$. Then, the relation (59) implies that the function $Y^{k}$ satisfies for all $0 \leq k \leq m, Y^{k}(z, T)=0$ for all $z \in[0, \infty)$. Consequently, the function $w_{m}^{\varepsilon}$ defined by 60 satisfies $w_{m}^{\varepsilon}(\cdot, T)=0$ on $[0,1]$. The result follows from the inequality $\left\|y^{\varepsilon}(\cdot, T)\right\|_{L^{2}(0,1)} \leq\left\|y^{\varepsilon}(\cdot, T)-w_{m}^{\varepsilon}(\cdot, T)\right\|_{L^{2}(0,1)}$ and Proposition 4.2. Figure 11.left illustrates this result.

This result should be compared with Lemma2.1. which asserts that the uncontrolled solution is exponentially small at any time $t>T^{\star}$. A gain of Proposition 5.1 is to construct smooth solutions from $t=0$, while the uncontrolled solution $y^{\varepsilon}$ belongs to $C^{\infty}([0,1] \times[\eta, T])$ for all $\eta>0 . y^{\varepsilon}$ belongs to $C^{\infty}([0,1] \times[0, T])$ if in addition the initial data satisfies regularity and compatibility assumptions (for the heat equation, $y_{0}^{\varepsilon} \in H^{k}(0,1)$, for all $k \in \mathbb{N}$ and $y_{0}^{\varepsilon}=\left(y_{0}^{\varepsilon}\right)^{(2 k)}=0$ at $x=0,1$ for all $k \in \mathbb{N}$, see Theorem 10.2 in [6] $)$.

Remark 13 From a controllability viewpoint, the null function is a much better approximate null control than the function $\sum_{k=0}^{m} \varepsilon^{k} v^{k}$ we construct in Proposition 5.1. The interest of such proposition (and of the results presented in the previous section) lies on the asymptotic of the solution of a direct problem. Given any function $v^{\varepsilon} \in L^{2}(0, T)$, we may approximate numerically the corresponding solution $y^{\varepsilon}$ and get an approximation, says $y_{h}^{\varepsilon}$, with $h$ is a parameter discretization. The error $\left\|y^{\varepsilon}-y_{h}^{\varepsilon}\right\|_{L^{2}\left(Q_{T}\right)}$ will be of the order $c_{\varepsilon} h^{\beta}$, for some rate $\beta>0$ (see [17]). For $\varepsilon$ small enough and $m$ large enough, this error will be larger than $\left\|y^{\varepsilon}-w_{m}^{\varepsilon}\right\|_{C\left([0, T], L^{2}(0,1)\right)}$. We emphasize that the function $w_{m}^{\varepsilon}$ is easily calculable because explicit for all $(x, t) \in Q_{T}$.

The limit case $T=1 / M$ can be considered as well but requires explicit formula. The function $w_{m}^{\varepsilon}(\cdot, T)$ no longer vanishes in this case. Let us consider for simplicity the case $m=0$ for which

$$
w_{0}^{\varepsilon}(x, T)=\mathcal{X}_{\varepsilon}(x) y^{0}(x, T)+\left(1-\mathcal{X}_{\varepsilon}(x)\right) Y^{0}\left(\frac{1-x}{\varepsilon}, T\right), \quad x \in(0,1) .
$$



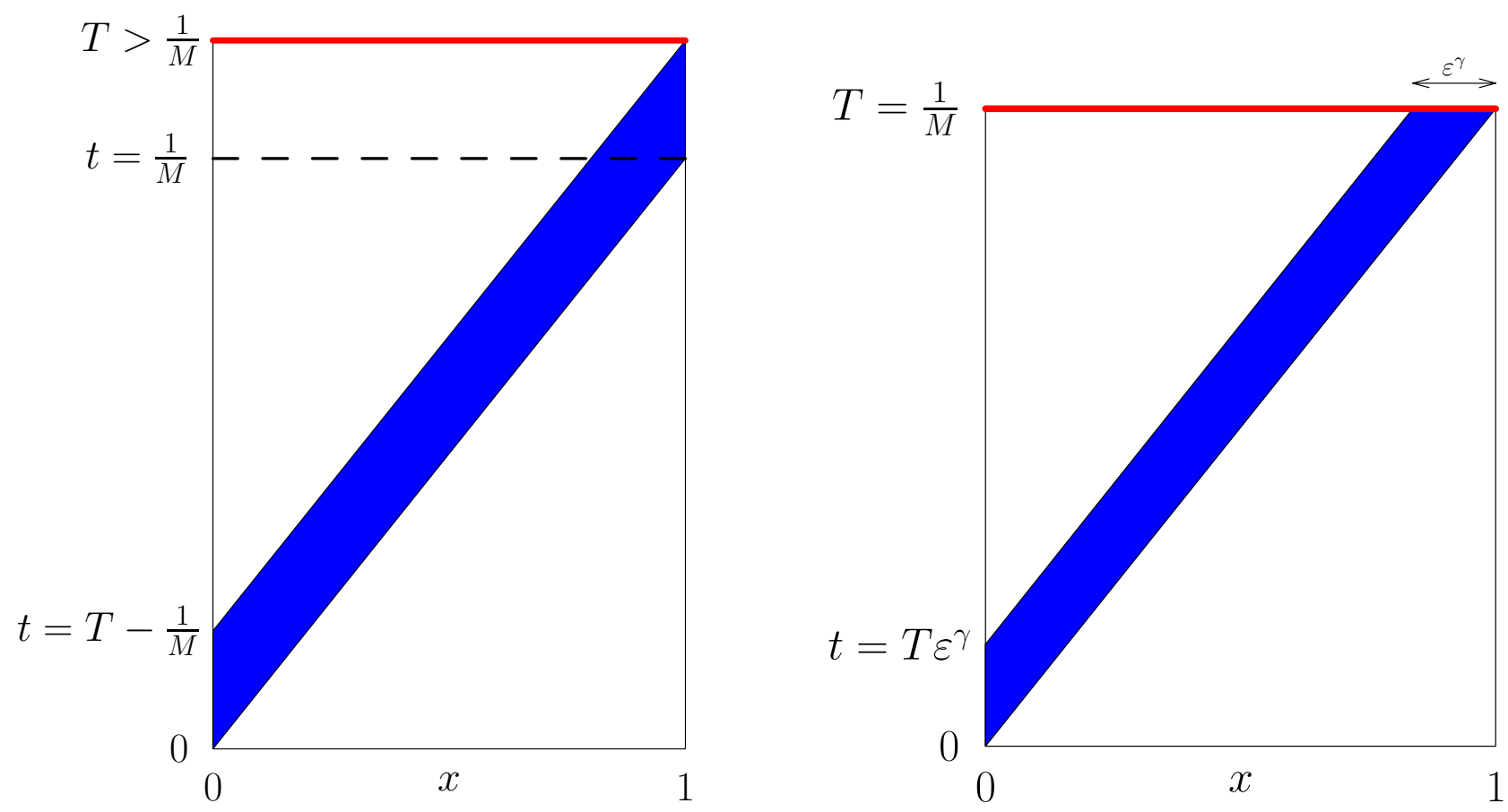

Figure 11: Influence zone of the control $v^{\varepsilon}$ (as $\left.\varepsilon \rightarrow 0\right)$ in $Q_{T}$ delimited by the characteristic line $M t-x=0$ for $T \geq \frac{L}{M}$ and $T=\frac{L}{M}$.

First, 43 leads to $Y^{0}\left(\frac{1-x}{\varepsilon}, T\right)=y_{0}(0)\left(1-e^{-\frac{M(1-x)}{\varepsilon}}\right)$. Therefore,

$$
\left\|\left(1-\mathcal{X}_{\varepsilon}(x)\right) Y^{0}\left(\frac{1-x}{\varepsilon}, T\right)\right\|_{L^{2}(0,1)}^{2}=\left(y_{0}(0)\right)^{2} \int_{0}^{1}\left(1-\mathcal{X}_{\varepsilon}(x)\right)^{2}\left(1-e^{-M z}\right)^{2} d x .
$$

Writing that $\left(1 \pm e^{-M z}\right) \leq 2$ and that $\left\|\left(1-\mathcal{X}_{\varepsilon}(x)\right)(1-x)^{p}\right\|_{L^{2}(0,1)}=\mathcal{O}\left(\varepsilon^{(2 p+1) \gamma / 2}\right), p \geq 0$, we obtain that

$$
\left\|\left(1-\mathcal{X}_{\varepsilon}(x)\right) Y^{0}\left(\frac{1-x}{\varepsilon}, T\right)\right\|_{L^{2}(0,1)}=\left|y_{0}(0)\right| \varepsilon^{\gamma / 2} .
$$

Moreover, from (37), we obtain, for all $x \in(0,1)$, that

$$
y^{0}(x, T)=v^{0}(\bar{x}), \quad \bar{x}:=\frac{1-x}{M}=T(1-x)
$$

and we may easily define a function $v^{0}$ such that the norm $\left\|\mathcal{X}_{\varepsilon} y^{0}(\cdot, T)\right\|_{L^{2}(0,1)}$ be equal to zero. Actually, since the function $\mathcal{X}_{\varepsilon}$ is supported in $\left[0,1-\varepsilon^{\gamma}\right]$, it suffices to take a function $v^{0}$ such that $v^{0}(\bar{x})=0$ for $x \in\left[0,1-\varepsilon^{\gamma}\right]$, i.e. supported in $\left[0, \varepsilon^{\gamma} T\right]$ (see Figure 11 right). Consequently, such control $v^{0}$ leads to

$$
\left\|w_{0}^{\varepsilon}(\cdot, T)\right\|_{L^{2}(0,1)} \leq\left|y_{0}(0)\right| \varepsilon^{\gamma / 2} .
$$

It remains to evaluate the term $\left\|y^{\varepsilon}(\cdot, T)-w_{0}^{\varepsilon}(\cdot, T)\right\|_{L^{2}(0,1)}$, equivalently the term $\left\|L^{\varepsilon} w_{0}^{\varepsilon}\right\|_{C\left([0, T], L^{2}(0,1)\right)}$. In order to satisfy the matching conditions of Lemma 4.1 , we define $v^{0}$ as follows

$$
v^{0}(t)=\sum_{p=0}^{1}(-1)^{p} \frac{(t M)^{p}}{p !}\left(y_{0}(0)\right)^{(p)} \mathcal{X}(t)
$$

for any $C^{1}([0, a],[0,1])$-function $\mathcal{X}$ such that $\mathcal{X}(0)=1,(\mathcal{X})^{k}(0)=0, \mathcal{X}(a)=0,(\mathcal{X})^{k}(a)=0, k=0,1$ with $\left.a \in] 0, \varepsilon^{\gamma} T\right]$. The function $v^{0}$ (and in particular the derivatives) depends on $\varepsilon$ here and so the constant $c_{m}$ in 68. 
Let us evaluate the first term $J_{\varepsilon}^{1}$ of $L_{\varepsilon} w_{0}^{\varepsilon}$ (see 69 ), restricted to $Q_{T}^{+}:=\left\{(x, t) \in Q_{T}, x-M t \leq 0\right\}$, in function of the support $[0, a]$ of $v^{0}$ : from $J_{\varepsilon}^{1}=-\varepsilon y_{x x}^{0}=-\frac{\varepsilon}{M^{2}}\left(v^{0}\right)^{(2)}(t-x / M)$, we have

$$
\begin{aligned}
\left\|J_{\varepsilon}^{1}\right\|_{L^{2}\left(Q_{T}^{+}\right)}^{2} & =\frac{\varepsilon^{2}}{M^{4}} \int_{0}^{1} \int_{\frac{x}{M}}^{T}\left(\left(v^{0}\right)^{(2)}(t-x / M)\right)^{2} d t d x \\
& =\frac{\varepsilon^{2}}{M^{4}} \int_{0}^{1} \int_{0}^{T-x / M}\left(\left(v^{0}\right)^{(2)}(t)\right)^{2} d t d x=\frac{\varepsilon^{2}}{M^{4}} \int_{0}^{1} \int_{0}^{\max (T-x / M, a)}\left(\left(v^{0}\right)^{(2)}(t)\right)^{2} d t d x \\
& \leq \frac{\varepsilon^{2}}{M^{4}} \int_{0}^{1} \int_{0}^{a}\left(\left(v^{0}\right)^{(2)}(t)\right)^{2} d t d x=\frac{1}{M^{4}}\left(\varepsilon\left\|\left(v^{0}\right)^{(2)}\right\|_{L^{2}(0, a)}\right)^{2} .
\end{aligned}
$$

Let us consider the polynomial of order 3 given by $\mathcal{X}(t)=1-3(t / a)^{2}+2(t / a)^{3}$ so that $\mathcal{X}(0)=1$ and $\mathcal{X}^{\prime}(0)=$ $\mathcal{X}(a)=\mathcal{X}^{\prime}(a)=0$ for all $a \neq 0$. Moreover, to simplify even more the computation, let assume that $y_{0}^{(1)}(0)=0$ so that the control $v^{0}$ is simply given by $v^{0}(t)=y_{0}(0) \mathcal{X}(t) 1_{[0, a]}(t)$ leading to $\left\|\left(v^{0}\right)^{(2)}\right\|_{L^{2}(0, a)}=\frac{12\left|y_{0}(0)\right|}{a^{3 / 2}}$ and then (from 93p)

$$
\left\|J_{\varepsilon}^{1}\right\|_{L^{2}\left(Q_{T}^{+}\right)} \leq \frac{12 \varepsilon}{a^{3 / 2} M^{2}}\left|y_{0}(0)\right| .
$$

We are therefore looking for $a \leq T \varepsilon^{\gamma}$ such that $\varepsilon^{2} / a^{3} \rightarrow 0$ as $\varepsilon \rightarrow 0$. We take $a=T \varepsilon^{\gamma^{\prime}}$. This requires $\gamma^{\prime} \in[\gamma, 2 / 3]$ and then $\left\|J_{\varepsilon}^{1}\right\|_{L^{2}\left(Q_{T}^{+}\right)} \leq \frac{12\left|y_{0}(0)\right|}{M^{2}} \varepsilon^{1-3 \gamma^{\prime} / 2} \leq \frac{12\left|y_{0}(0)\right|}{T^{3 / 2} M^{2}} \varepsilon^{1-3 \gamma / 2}$.

We can proceed in a similar way with the other terms in $(69)$ and determine a rate $\tau=\tau(\gamma)$ such that $\left\|L_{\varepsilon}\left(w_{0}^{\varepsilon}\right)\right\|_{L^{2}\left(Q_{T}\right)} \leq c \varepsilon^{\tau}$ and then $\left\|y^{\varepsilon}(\cdot, T)-w_{0}^{\varepsilon}(\cdot, T)\right\|_{L^{2}(0,1)} \leq c \varepsilon^{\tau}$. This allows to conclude that there exists a control function $v^{0} \in C^{1}\left(\left[0, T \varepsilon^{\gamma}\right]\right)$ such that the solution of $(34)$ with $v^{\varepsilon}=v^{0}$ satisfies

$$
\left\|y^{\varepsilon}(\cdot, T)\right\|_{L^{2}(0,1)} \leq\left|y_{0}(0)\right|\left(c \varepsilon^{\tau(\gamma)}+\varepsilon^{\gamma / 2}\right),
$$

with $\gamma<2 / 3$ (instead of $\gamma<1$ in Proposition 5.1). This stronger condition shows how the convergence is affected in the limit case $T=1 / M$. Nevertheless, after tedious computations, we may extend this construction of $v^{0}$ to any order $k$ and improve the rate in the estimate 94 . This may allow to obtain a better estimate that in the uncontrolled case discussed in Lemma 2.1. Remark that in the uncontrolled case, the norm $\left\|y^{\varepsilon}(\cdot, T)\right\|_{L^{2}(0,1)}$ is a priori not exponentially small for $T=1 / M$.

\subsection{Conclusions of the asymptotic analysis}

We have derived an asymptotic expansion at any order $m$ of the solution $y^{\varepsilon}$ of an advection-diffusion equation with respect to the diffusion parameter $\varepsilon$. The matching asymptotic expansion method allows to describe the boundary layer of the solution at the right extremity of the interval. As is usual, the asymptotic analysis requires the initial and boundary conditions to be regular enough. This is not restrictive as $y^{\varepsilon}$ solves a parabolic type equation. In an essential way, we have also assumed compatibility equations between these conditions at the point $(x, t)=(0,0)$ where the main characteristic of equation $x-M t=0$ starts. This allows to get rid off the boundary layer for $y^{\varepsilon}$ on this characteristic. This also allows to obtain a regular approximation $w_{m}^{\varepsilon}$ of $y^{\varepsilon}$ so that the norm $\left\|y^{\varepsilon}-w_{m}^{\varepsilon}\right\|_{C\left([0, T], L^{2}(0,1)\right)}$ is of size $\mathcal{O}\left(\varepsilon^{\frac{2 m+1}{2}} \gamma\right)$. As expected, the approximation $w_{m}^{\varepsilon}$ is the sum of $m+1$ explicit solutions of transport equations plus an initial layer corrector, exponentially small, which retains the parabolic character of the initial equation. The approximation $w_{m}^{\varepsilon}$ is useful to construct explicit and regular approximate null controls for $y^{\varepsilon}$ as soon as the controllability time satisfies $T \geq 1 / M$. Moreover, for a precise class of initial conditions, this decomposition of the solution $y^{\varepsilon}$ remains true, uniformly with respect to $m$. This allows to reduce the null controllability of $y^{\varepsilon}$ to the null controllability of the corrector, a priori easier since this latter is exponentially small with respect to $\varepsilon$.

The knowledge of the minimal uniform controllability time $T_{M}$ remains unknown for an arbitrary initial condition. One may use our asymptotic analysis in the optimality system (33) which characterizes the unique control of minimal $L^{2}(0, T)$-norm, $T, \varepsilon$ and the initial condition $y_{0}$ (assumed independent of $\varepsilon$ ) being fixed. Let us focus on the optimality equation $v^{\varepsilon}(t)=\varepsilon \varphi_{x}^{\varepsilon}(0, t)$ which links the forward and backward solutions. Using the inner expansion for $\varphi^{\varepsilon}$ (see Section 4.1.5), this equality rewrites as follows

$$
v^{0}(t)+\varepsilon v^{1}(t)+\cdots=\Phi_{z}^{0}(0, t)+\varepsilon \Phi_{z}^{1}(0, t)+\cdots, \quad \forall t \in(0, T) .
$$


At the zero order, we get therefore the equality $v^{0}(t)=\Phi_{z}^{0}(0, t)$ leading, using 87 and 89 simultaneously, to

$$
v^{0}(t)=M \varphi^{0}(0, t)= \begin{cases}M \varphi_{T}^{0}(M(T-t)), & t \in] T-1 / M, T], \\ 0, & t \in[0, T-1 / M] .\end{cases}
$$

The function $\varphi^{0}$ defined in $Q_{T}$ is given by (87). If $T>1 / M$, the last equality contradicts the matching conditions (61), notably $v^{0}(0)=y_{0}(0)$, unless that $y_{0}(0)=0$ ! If $T=1 / M$, we have $v^{0}(t)=M \varphi_{T}^{0}(1-M t), t \in[0,1 / M]$ and in particular $v^{0}(0)=\varphi_{T}^{0}(1)$. But again, this contradicts $(92)$ unless $v^{0}(0)=0$ (and so $\left.y_{0}(0)=0\right)$.

If we do not assume $y_{0}(0)=0$, then (95) leads to incompatibility and our asymptotic analysis is not effective to address the optimality system (33). To avoid this difficulty, we must relax the matching conditions (62) and (92) and therefore take into account the second boundary layer occurring for $y^{\varepsilon}$ and $\varphi^{\varepsilon}$ on the characteristic lines $\left\{(x, t) \in Q_{T}, L x-M t=0\right\}$ and $\left\{(x, t) \in Q_{T}, L x-M(t-T)-1=0\right\}$ respectively. This is done in [2]. Briefly, this layer, of size $\mathcal{O}(\sqrt{\varepsilon})$, leads to the introduction of a second inner expansion of the form

$$
\sum_{k=0}^{m} \varepsilon^{\frac{k}{2}} W^{k / 2}(w, t), \quad w=\frac{x-M t}{\sqrt{\varepsilon}} \in\left(-\frac{M t}{\sqrt{\varepsilon}}, \frac{1-M t}{\sqrt{\varepsilon}}\right), t \in(0, T) .
$$

Putting this expansion into equation $(34)_{1}$, the identification of the powers of $\varepsilon$ yields

$$
W_{t}^{k / 2}(w, t)-W_{w w}^{k / 2}(w, t)=0, \quad \text { for any } 0 \leq k \leq m,
$$

so that at the first order, taking into account the matching conditions with the outer expansion, $W^{0}$ solves the boundary value problem

$$
\left\{\begin{array}{lr}
W_{t}^{0}(w, t)-W_{w w}^{0}(w, t)=0, & (w, t) \in \mathbb{R} \times(0, T), \\
\lim _{w \rightarrow+\infty} W^{0}(w, t)=\lim _{x \rightarrow(M t)^{+}} y^{0}(x, t):=y^{0}\left((M t)^{+}, t\right)=y_{0}(0), & t \in(0, T), \\
\lim _{w \rightarrow-\infty} W^{0}(w, t)=\lim _{x \rightarrow(M t)^{-}} y^{0}(x, t):=y^{0}\left((M t)^{-}, t\right)=v^{0}(0), & t \in(0, T),
\end{array}\right.
$$

and is given by

$$
W^{0}(w, t)=\operatorname{erf}\left(\frac{w}{2 \sqrt{t}}\right) \frac{y_{0}(0)-v^{0}(0)}{2}+\frac{y_{0}(0)+v^{0}(0)}{2} .
$$

$W^{0}$ makes appear the jump $y^{0}(0)-v^{0}(0)$ (which does not have to vanish anymore) of the solution $y^{\varepsilon}$ at the first order across the characteristics $\left\{(x, t) \in Q_{T}, x-M t=0\right\}$. Localized around such characteristics, $W^{0}$ enriches the first order approximation $w_{0}^{\varepsilon}$ given by $(60)$ (see also $\widetilde{w}_{0}^{\varepsilon}$ defined in Remark 10 ).

The next step will be to address the asymptotic analysis of the system 33 . This requires to adapt this work in order to treat initial conditions which depends on $\varepsilon$ and controls which are only in $L^{2}(0,1)$, as done in 9 for a fourth order partial differential equation.

\section{Concluding remarks}

In spite of its apparent simplicity, the advection-diffusion equation $y_{t}-\varepsilon y_{x x}+M y_{x}=0$ leads to challenging open problems from an exact controllability viewpoint. The evaluation, either theoretical or numerical of the corresponding cost of control as the diffusion coefficient $\varepsilon$ goes to zero remains open. This is mainly due to the fact that the uncontrolled solution is exponentially small with respect to $\varepsilon$ as soon as the time $t$ is strictly larger than $L /|M|$. Consequently, the gap between exact and approximate controllability is huge as $\varepsilon$ goes to zero. For this reason, for small values of $\varepsilon$, any direct numerical approach can not capture the behavior of the cost of control. Computations for intermediate values give however some hints on the structure of the worst initial conditions. On the other hand, the asymptotic analysis with respect to $\varepsilon$ allows to decompose the solution to be controlled as the sum of a pure advection part plus an advection-diffusion component, exponentially small with respect to $\varepsilon$. Provided some regularity conditions on the controls and on the initial data, this allows to report the controllability issue on the small advection-diffusion component, leading, for $M>0$ to $T_{M}=1 / M$ 
(for $M>0$ ). These regularity conditions on the data, which are intrinsic to the asymptotic method used, are however quite strong. Consequently, for an arbitrary initial condition $y_{0}^{\varepsilon} \in L^{2}(0,1)$, the precise value of the uniform minimal time $T_{M}$, remains, as far as we know, an open question. The negative case $M<0$ seems, on a numerical viewpoint, out of reach, at its leads to very oscillating control near the controllability time. On the other hand, from an asymptotic analysis viewpoint, it seems more accessible, since, roughly, the initial condition and the support of the control live on the same side of the main characteristic line.

\section{Annexe}

7.1 Tables of cost of control $K_{h}(\varepsilon, T, M)$

\begin{tabular}{|c|c|c|c|c|}
\hline$\varepsilon$ & $T=0.95$ & $T=0.99$ & $T=1$. & $T=1.05$ \\
\hline $10^{-3}$ & 237.877 & 30.4972 & 18.7555 & 2.2915 \\
$1.25 \times 10^{-3}$ & 190.574 & 29.7622 & 19.1953 & 2.8028 \\
$1.5 \times 10^{-3}$ & 159.813 & 29.0015 & 19.3883 & 3.2556 \\
$1.75 \times 10^{-3}$ & 138.166 & 28.2446 & 19.4234 & 3.6529 \\
$2 \times 10^{-3}$ & 122.044 & 27.4997 & 19.3540 & 4.0005 \\
$2.25 \times 10^{-3}$ & 109.519 & 26.7745 & 19.2093 & 4.3013 \\
$2.5 \times 10^{-3}$ & 99.476 & 26.0722 & 19.0163 & 4.5623 \\
$3 \times 10^{-3}$ & 84.250 & 24.7318 & 18.5275 & 4.9814 \\
$4 \times 10^{-3}$ & 64.648 & 22.3060 & 17.3600 & 5.5078 \\
$5 \times 10^{-3}$ & 52.289 & 20.1837 & 16.1269 & 5.7530 \\
$6 \times 10^{-3}$ & 43.650 & 18.3289 & 14.9392 & 5.8259 \\
$7 \times 10^{-3}$ & 37.213 & 16.6883 & 13.8166 & 5.7787 \\
$8 \times 10^{-3}$ & 32.198 & 15.2461 & 12.7839 & 5.6683 \\
$9 \times 10^{-3}$ & 28.210 & 13.9660 & 11.8380 & 5.5099 \\
$10^{-2}$ & 24.934 & 12.8331 & 10.9763 & 5.3276 \\
$1.25 \times 10^{-2}$ & 18.898 & 10.5015 & 9.1493 & 4.8282 \\
$1.5 \times 10^{-2}$ & 14.810 & 8.7281 & 7.7087 & 4.3378 \\
$1.75 \times 10^{-2}$ & 11.913 & 7.3526 & 6.5694 & 3.8897 \\
$2 \times 10^{-2}$ & 9.784 & 6.2780 & 5.6566 & 3.4943 \\
$2.25 \times 10^{-2}$ & 8.176 & 5.4196 & 4.9210 & 3.1506 \\
$2.5 \times 10^{-2}$ & 6.937 & 4.7293 & 4.3237 & 2.8534 \\
$3 \times 10^{-2}$ & 5.180 & 3.7047 & 3.4240 & 2.3744 \\
$4 \times 10^{-2}$ & 3.264 & 2.4895 & 2.3297 & 1.7350 \\
$5 \times 10^{-2}$ & 2.294 & 1.8261 & 1.7304 & 1.3416 \\
$6 \times 10^{-2}$ & 1.736 & 1.4209 & 1.3522 & 1.0848 \\
$7 \times 10^{-2}$ & 1.376 & 1.1510 & 1.1030 & 0.8978 \\
$8 \times 10^{-2}$ & 1.113 & 0.9596 & 0.9223 & 0.7612 \\
$9 \times 10^{-2}$ & 0.0952 & 0.8130 & 0.7865 & 0.6554 \\
$10^{-1}$ & 0.8175 & 0.7075 & 0.6808 & 0.5711 \\
\hline
\end{tabular}

\begin{tabular}{|c|c|}
\hline$\varepsilon$ & $T=1$. \\
\hline $10^{-3}$ & 10718.0955 \\
$1.25 \times 10^{-3}$ & 13839.4039 \\
$1.5 \times 10^{-3}$ & 16903.9918 \\
$1.75 \times 10^{-3}$ & 19898.1360 \\
$2 \times 10^{-3}$ & 22812.2634 \\
$2.25 \times 10^{-3}$ & 25638.7601 \\
$2.5 \times 10^{-3}$ & 28375.3693 \\
$3 \times 10^{-3}$ & 33575.9482 \\
$4 \times 10^{-3}$ & 42871.1424 \\
$5 \times 10^{-3}$ & 50751.4443 \\
$6 \times 10^{-3}$ & 57316.7716 \\
$7 \times 10^{-3}$ & 62692.7273 \\
$8 \times 10^{-3}$ & 66997.3602 \\
$9 \times 10^{-3}$ & 70350.3966 \\
$10^{-2}$ & 72862.0738 \\
$1.25 \times 10^{-2}$ & 76089.8839 \\
$1.5 \times 10^{-2}$ & 75988.4041 \\
$1.75 \times 10^{-2}$ & 73579.1022 \\
$2 \times 10^{-2}$ & 69647.3042 \\
$2.25 \times 10^{-2}$ & 64735.7778 \\
$2.5 \times 10^{-2}$ & 59254.0430 \\
$3 \times 10^{-2}$ & 47994.1519 \\
$4 \times 10^{-2}$ & 27872.8642 \\
$5 \times 10^{-2}$ & 13312.4452 \\
$6 \times 10^{-2}$ & 5687.6960 \\
$7 \times 10^{-2}$ & 1864.7252 \\
$8 \times 10^{-2}$ & 648.7029 \\
$9 \times 10^{-2}$ & 264.5594 \\
$10^{-1}$ & 123.3069 \\
\hline
\end{tabular}

Cost of control $K_{h}(\varepsilon, T, M)$ with respect to $T$ and $\varepsilon$ for $M=1$ (Left) and $M=-1$ (Right); $h=1 / 320-r=h^{2}$ $-\beta=10^{-16}$.

\subsection{Free-Fem++ code}

1 border bas $(s=0,1)\{x=s ; y=0 ; l a b e l=N t o p ;\} ; \quad$ border $\operatorname{droit}(s=0, T)\{x=1 ; y=s ; l a b e l=N r i g h t ;\}$ 2 border haut $(\mathrm{s}=1,0)\{\mathrm{x}=\mathrm{s} ; \mathrm{y}=\mathrm{T} ; \mathrm{label}=$ Nhaut $\}$ border gauche $(\mathrm{s}=\mathrm{T}, 0)\{\mathrm{x}=0 ; \mathrm{y}=\mathrm{s} ; \mathrm{label}=$ Ngauche $\}$ 3 mesh Th=buildmesh (bas (50)+droit (50)+haut (50)+gauche (50)) ; 4 


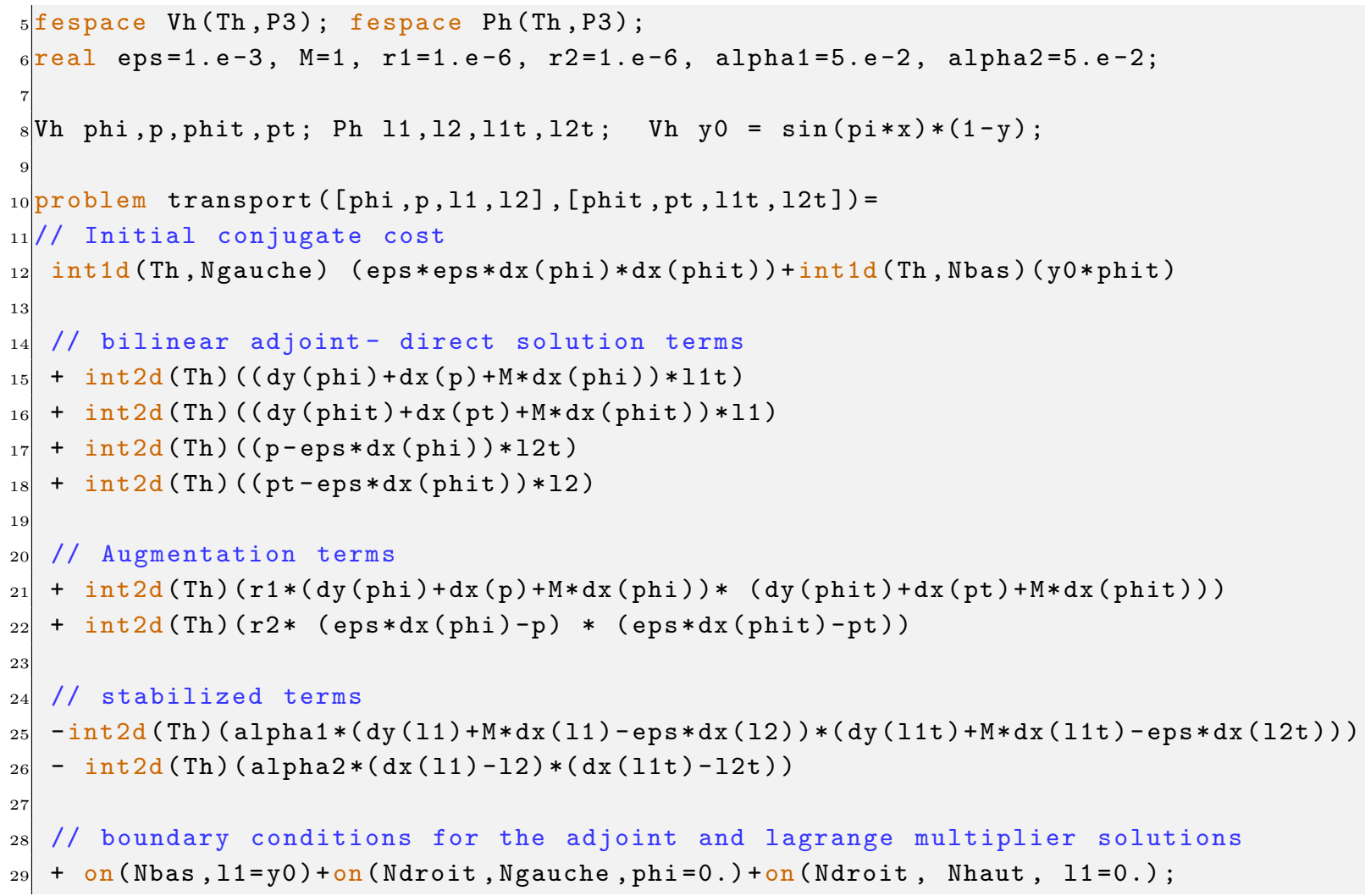

\section{References}

[1] Y. Amirat And A. MÜNCh, Asymptotic analysis of an advection-diffusion equation and application to boundary controllability, Preprint available at https://hal-clermont-univ.archives-ouvertes.fr/hal-01654390, (2017).

[2] —_ Asymptotic analysis of an advection-diffusion equation with respect to the diffusion coefficient: description of the boundary layers, In preparation, (2018).

[3] H. J. C. Barbosa and T. J. R. Hughes, The finite element method with Lagrange multipliers on the boundary: circumventing the Babuška-Brezzi condition, Comput. Methods Appl. Mech. Engrg., 85 (1991), pp. 109-128.

[4] F. Ben Belgacem and S. M. Kaber, On the Dirichlet boundary controllability of the one-dimensional heat equation: semi-analytical calculations and ill-posedness degree, Inverse Problems, 27 (2011), pp. $055012,19$.

[5] F. BoYer, On the penalized HUM approach and its applications to the numerical approximation of nullcontrols for parabolic problems, in CANUM 2012, 41e Congrès National d'Analyse Numérique, vol. 41 of ESAIM Proc., EDP Sci., Les Ulis, 2013, pp. 15-58.

[6] H. Brezis, Functional analysis, Sobolev spaces and partial differential equations, Universitext, Springer, New York, 2011.

[7] F. Brezzi And M. Fortin, Mixed and hybrid finite element methods, vol. 15 of Springer Series in Computational Mathematics, Springer-Verlag, New York, 1991.

[8] C. Carthel, R. Glowinski, and J.-L. Lions, On exact and approximate boundary controllabilities for the heat equation: a numerical approach, J. Optim. Theory Appl., 82 (1994), pp. 429-484. 
[9] C. CAstro And A. MÜNCH, Exact null controllability of a rayleigh beam: asymptotic analysis with respect to the thickness, In preparation, (2018).

[10] D. Chapelle And K.-J. Bathe, The inf-sup test, Comput. \& Structures, 47 (1993), pp. 537-545.

[11] F. Chatelin, Eigenvalues of matrices, vol. 71 of Classics in Applied Mathematics, Society for Industrial and Applied Mathematics (SIAM), Philadelphia, PA, 2012. With exercises by Mario Ahués and the author, Translated with additional material by Walter Ledermann, Revised reprint of the 1993 edition [ MR1232655].

[12] P. G. Ciarlet, The finite element method for elliptic problems, vol. 40 of Classics in Applied Mathematics, Society for Industrial and Applied Mathematics (SIAM), Philadelphia, PA, 2002. Reprint of the 1978 original [North-Holland, Amsterdam; MR0520174 (58 \#25001)].

[13] N. Cîndea And A. Münch, A mixed formulation for the direct approximation of the control of minimal $L^{2}$-norm for linear type wave equations, Calcolo, 52 (2015), pp. 245-288.

[14] J.-M. Coron, Control and nonlinearity, vol. 136 of Mathematical Surveys and Monographs, American Mathematical Society, Providence, RI, 2007.

[15] J.-M. Coron And S. Guerrero, Singular optimal control: a linear 1-D parabolic-hyperbolic example, Asymptot. Anal., 44 (2005), pp. 237-257.

[16] J. Dardé and S. Ervedoza, On the Reachable Set for the One-Dimensional Heat Equation, SIAM J. Control Optim., 56 (2018), pp. 1692-1715.

[17] P. Deuring, R. Eymard, And M. Mildner, $L^{2}$-stability independent of diffusion for a finite elementfinite volume discretization of a linear convection-diffusion equation, SIAM J. Numer. Anal., 53 (2015), pp. 508-526.

[18] E. Fernández-Cara, M. González-Burgos, and L. De Teresa, Boundary controllability of parabolic coupled equations, J. Funct. Anal., 259 (2010), pp. 1720-1758.

[19] E. Fernández-CARA And A. Münch, Numerical exact controllability of the $1 D$ heat equation: duality and Carleman weights, J. Optim. Theory Appl., 163 (2014), pp. 253-285.

[20] A. V. Fursikov and O. Y. Imanuvilov, Controllability of evolution equations, vol. 34 of Lecture Notes Series, Seoul National University Research Institute of Mathematics Global Analysis Research Center, Seoul, 1996.

[21] O. GLass, A complex-analytic approach to the problem of uniform controllability of a transport equation in the vanishing viscosity limit, Journal of Functional Analysis, 258 (2010), pp. 852-868.

[22] S. Guerrero and G. Lebeau, Singular optimal control for a transport-diffusion equation, Comm. Partial Differential Equations, 32 (2007), pp. 1813-1836.

[23] F. Hecht, New development in freefem++, J. Numer. Math., 20 (2012), pp. 251-265.

[24] J. Kevorkian and J. D. Cole, Multiple scale and singular perturbation methods, vol. 114 of Applied Mathematical Sciences, Springer-Verlag, New York, 1996.

[25] S. G. Krantz And H. R. Parks, A primer of real analytic functions, Birkhäuser Advanced Texts: Basler Lehrbücher. [Birkhäuser Advanced Texts: Basel Textbooks], Birkhäuser Boston, Inc., Boston, MA, second ed., 2002.

[26] S. LABbÉ And E. TRÉLAT, Uniform controllability of semidiscrete approximations of parabolic control systems, Systems Control Lett., 55 (2006), pp. 597-609. 
[27] G. Lebeau and L. Robbiano, Contrôle exact de l'équation de la chaleur, Comm. Partial Differential Equations, 20 (1995), pp. 335-356.

[28] J.-L. Lions, Perturbations singulières dans les problèmes aux limites et en contrôle optimal, Lecture Notes in Mathematics, Vol. 323, Springer-Verlag, Berlin-New York, 1973.

[29] P. LIssy, A link between the cost of fast controls for the 1-d heat equation and the uniform controllability of a 1-d transport-diffusion equation, Comptes Rendus Mathematique, 350 (2012), pp. 591-595.

[30] — Explicit lower bounds for the cost of fast controls for some 1-D parabolic or dispersive equations, and a new lower bound concerning the uniform controllability of the 1-D transport-diffusion equation, J. Differential Equations, 259 (2015), pp. 5331-5352.

[31] L. Miller, The control transmutation method and the cost of fast controls, SIAM J. Control Optim., 45 (2006), pp. 762-772.

[32] A. MüNCH, Inverse problems for linear parabolic equations using mixed formulations - Part 2 : Numerical analysis, In preparation, (2018).

[33] A. MüNCH, Numerical estimation of the cost of boundary controls for the equation $y_{t}-\varepsilon y_{x x}+M y_{x}=0$ with respect to $\varepsilon$, To appear in the SEMA-SIMAI springer series - Preprint available at https://hal.archivesouvertes.fr/hal-01496856, (2018).

[34] A. MÜnch And S. Montaner, Approximation of controls for linear wave equations: a first order mixed formulation, Preprint available at https://hal.archives-ouvertes.fr/UMR6620/hal-01792949v1, (2018).

[35] A. MÜnch And D. A. SouzA, A mixed formulation for the direct approximation of $L^{2}$-weighted controls for the linear heat equation, Adv. Comput. Math., 42 (2016), pp. 85-125.

[36] A. Münch AND D. A. SouzA, Inverse problems for linear parabolic equations using mixed formulationsPart 1: Theoretical analysis, J. Inverse Ill-Posed Probl., 25 (2017), pp. 445-468.

[37] A. MÜNCh And E. Zuazua, Numerical approximation of null controls for the heat equation: ill-posedness and remedies, Inverse Problems, 26 (2010), pp. 085018, 39.

[38] Y. OU AND P. ZHU, The vanishing viscosity method for the sensitivity analysis of an optimal control problem of conservation laws in the presence of shocks, Nonlinear Anal. Real World Appl., 14 (2013), pp. 1947-1974.

[39] J. Sanchez Hubert and E. SÁnchez-Palencia, Vibration and coupling of continuous systems, SpringerVerlag, Berlin, 1989. Asymptotic methods.

[40] M. Van Dyke, Perturbation methods in fluid mechanics, The Parabolic Press, Stanford, Calif., annotated ed., 1975. 